\title{
Einstein Geometric Aggregation Operators using a Novel Complex Interval-valued Pythagorean Fuzzy Setting with Application in Green Supplier Chain Management
}

\author{
Zeeshan Ali ${ }^{1}$, Tahir Mahmood ${ }^{1}$, Kifayat Ullah ${ }^{2}$, Qaisar Khan ${ }^{3}$ \\ ${ }^{1}$ Department of Mathematics \& Statistics, International Islamic University Islamabad, Pakistan, e-mail: \\ zeeshanalinsr@gmail.com; tahirbakhat@iiu.edu.pk; \\ ${ }^{2}$ Department of Mathematics, Riphah International University, Lahore Campus, Pakistan, e-mail: \\ kifayat.khan.dr@gmail.com; \\ ${ }^{3}$ Department of Mathematics, University of Huripur, Pakistan, e-mail: qaisar.khan@uoh.edu.pk;
}

\begin{tabular}{l}
\hline \hline Article Info \\
\hline Article history: \\
Received May 5, 2021 \\
Revised June 6, 2021 \\
Accepted June 9, 2021 \\
\hline
\end{tabular}

\section{Keywords:}

Complex interval-valued;

Pythagorean fuzzy sets;

Generalized Geometric

aggregation operators;

Einstein t-norm and t-conorm;

Multicriteria decision-making.

\begin{abstract}
The principle of a complex interval-valued Pythagorean fuzzy set (CIVPFS) is a valuable procedure to manage inconsistent and awkward information genuine life troubles. The principle of CIVPFS is a mixture of the two separated theories such as complex fuzzy set and interval-valued Pythagorean fuzzy set which covers the truth grade (TG) and falsity grade (FG) in the form of the complex number whose real and unreal parts are the sub-interval of the unit interval. The superiority of the CIVPFS is that the sum of the square of the upper grade of the real part (also for an unreal part) of the duplet is restricted to the unit interval. The goal of this article is to explore the new principle of CIVPFS and its algebraic operational laws. By using the CIVPFSs, certain Einstein operational laws by using the t-norm and t-conorm are also developed. Additionally, we explore the complex intervalvalued Pythagorean fuzzy Einstein weighted geometric (CIVPFEWG), complex interval-valued Pythagorean fuzzy Einstein ordered weighted geometric (CIVPFEOWG) operators and utilized their special cases. Moreover, a multicriteria decision-making (MCDM) technique is explored based on the elaborated operators by using the complex interval-valued Pythagorean fuzzy (CIVPF) information. To determine the consistency and reliability of the elaborated operators, we illustrated certain examples by using the explored principles. Finally, to determine the supremacy and dominance of the explored theories, the comparative analysis and graphical expressions of the developed principles are also discussed.
\end{abstract}

Copyright (C) 2020 Regional Association for Security and crisis management and European centre for operational research. All rights reserved.

\section{Corresponding Author:}

Tahir Mahmood,

Department of Mathematics \& Statistics, International Islamic University Islamabad, Pakistan.

Email: tahirbakhat@iiu.edu.pk

\section{Introduction}

Because of the expanding intricacy of the framework, it is hard for the leader to choose the best other option/object from a family of appealing choices. Be that as it may, it is difficult, to sum up, yet it is not staggering to accomplish the best single objective. Countless MCDM issues exist in decision-making, where the rules are found to be dubious, equivocal, loose, and obscure. Therefore, the fresh set gives off an impression of being inadequate in managing this vulnerability and imprecision in the information and can be handily managed by utilizing fuzzy data. To manage such vulnerability also, vagueness, the principle of the fuzzy set (FS) was elaborated by Zadeh (1965). FS gives the truth grade (TG) against the value which is 
taken from the set of attributes is limited to the unit interval. After their successful utilization, certain scholars have employed it in the natural environment of separated areas. For example, interval-valued FS was explored by Zadeh (1975), Torra (2010) elaborated the principle of hesitant FS (HFS), Karnik and Mendel (2001) utilized certain operational laws based on type-2 FS (T-2FS), Mendel and John (2002) developed T2FS made simple, and Mahmood (2020) developed the theory of bipolar soft sets.

The principle of FS has been utilized in separated areas, but the principle of FS has limited applications due to its structure. Because if a person faced information in the form of TG and falsity grade (FG), then the principle of FS has been failed in certain actual life troubles. To conquer this deficiency and adjust to the uninterrupted complicacy of certain actual life troubles, a type of intuitionistic FS (IFS) was implemented at Atanassov (1986). IFS gives the information's in the form of TG and FG against the value which is taken from the set of attributes with a rule that is the sum duplet is limited to the unit interval. After their successful utilization, certain scholars have employed it in the natural environment of separated areas. For example, Atanassov (1989) developed the interval-valued IFS and their application's; Garg and Rani (2021) explored similarity measures based on the transformed right-angle tringles among IFSs, Ejegwa and Onyeke (2021) intuitionistic fuzzy statistical correlation algorithm, Xue et al. (2021) utilized the measure-based belief function by using the IFSs, Aydin and Enginoglu (2021) proposed interval-valued intuitionistic fuzzy parametrized interval-valued intuitionistic fuzzy soft sets, Huang et al. (2021) developed the complete ranking method for interval-valued IFSs, and Ecer and Pamucer (2021) initiated the MARCOS method for IFSs.

The principle of IFS has been utilized in separated areas, but the principle of IFS has limited applications due to its structure. Because if a person faced information in the form of TG and falsity grade (FG) with a condition that is the sum of duplet is exceeded from the unit interval, then the principle of IFS has been failed in certain actual life troubles. To conquer this deficiency and adjust to the uninterrupted complicacy of certain actual life troubles, a type of Pythagorean FS (PFS) was implemented by Yager (2013). PFS gives the information's in the form of TG and FG against the value which is taken from the set of attributes with a rule that is the sum of the squares of duplet is limited to the unit interval. After their successful utilization, certain scholars have employed it in the natural environment of separated areas. For example, Garg (2016) explored interval-valued PFSs and their application's, Ayyildiz and Gumus (2021) utilized the AHP method based on interval-valued PFSs, Ejegwa et al. (2021) implemented the correlation measures by using the PFSs, Zhao et al. (2021) explored TODIM method for interval-valued PFSs, Gao et al. (2021) developed the quantum Pythagorean fuzzy evidence theory, Pan et al. (2021) proposed similarity measures for PFSs, Zulqarnain et al. (2021) initiated the TOPSIS method for Pythagorean fuzzy hyper-soft sets, Rani et al. (2021) developed the weighted discrimination based approximation approach by using the PFSs, Calik (2021) initiated the AHP and TOPSIS method for PFSs and discussed their application in green supplier chain management, Chen (2021) developed the likelihood-based optimization based on PFSs.

All the prevailing theories based on FS and their modifications, inconsistency, and uncertainties are involved in the form of TG whose value is also in the form of real numbers and certain user information may be lost and the decision-maker is affected by this. To manage such vulnerability, the principle of complex FS (CFS) was elaborated by Ramot et al. (2002). CFS gives the TG against the value which is taken from the set of attributes in the form of a complex number whose real and unreal parts are limited to the unit interval. After their successful utilization, certain scholars have employed it in the natural environment of separated areas. The principle of CFS has been utilized in separated areas, but the principle of CFS has limited applications due to its structure. Because if a person faced information in the form of TG and FG, then the principle of CFS has been failed in certain actual life troubles. To conquer this deficiency and adjust to the uninterrupted complicacy of certain actual life troubles, a type of complex IFS (CIFS) was implemented Alkouri and Salleh (2012). CIFS gives the information's in the form of TG and FG against the value which is taken from the set of attributes with a rule that is the sum of the real part (also for the imaginary part) of duplet is limited to the unit interval. After their successful utilization, certain scholars have employed it in the natural environment of separated areas. For example, Garg and Rani (2019a) initiated the complex intervalvalued IFSs and their application's, Garg and Rani (2019b) explored the information measures for CIFSs, Ali et al. (2016) proposed complex intuitionistic fuzzy classes, Garg and Rani (2019c) initiated the correlation coefficient for CIFSs, Kumar, and Bajaj (2014) developed distance measures by using the complex intuitionistic fuzzy soft sets, Garg and Rani (2019d) elaborated the generalized geometric aggregation operators for CIFSs, Ngan et al. (2020) proposed quaternion numbers based on CIFSs, Rani and Garg [35] explored the power aggregation operators based on CIFSs.

The principle of CIFS has been utilized in separated areas, but the principle of CIFS has limited applications due to its structure. Because if a person faced information in the form of TG and FG with a condition that is the sum of the real part (also for the imaginary part) of the duplet is exceeded from the unit interval, then the principle of CIFS has been failed in certain actual life troubles. To conquer this deficiency 
and adjust to the uninterrupted complicacy of certain actual life troubles, a type of complex PFS (CPFS) was implemented Ullah et al. (2020). CPFS gives the information's in the form of TG and FG against the value which is taken from the set of attributes with a rule that is the sum of the squares of the real part (also for the imaginary part) of the duplet is limited to the unit interval. After their successful utilization, certain scholars have employed it in the natural environment of separated areas. But no one proposed the theory of complex interval-valued Pythagorean fuzzy set (CIVPFS) and utilized any measures, operators, and methods in the environment of CIVPFSs. Ullah et al. (2020) only explored some measures based on CPFSs, but to determine the relationship among any number of attributes when they are in the form of interval-valued, then the existing theories have been failed, similarly, aggregation operators is one of the important techniques to manage inconsistent and unreliable information in genuine life troubles. For this, we explored some Einstein operational laws by using the novel approach of CIVPFSs. The main goals of this manuscript are discussed below.

1. To propose the novel approach of CIVPFSs and their algebraic laws.

2. To explore certain Einstein operational laws by using the t-norm and t-conorm based on CIVPFSs.

3. To explore the CIVPFEWG, CIVPFEOWG operators, and utilized their special cases by using the elaborated Einstein laws.

4. To explore an MCDM technique based on the elaborated operators by using the CIVPF information.

5. To determine the consistency and reliability of the elaborated operators, we illustrated certain examples by using the explored principles.

6. To determine the supremacy and dominance of the explored theories, the comparative analysis and graphical expressions of the developed principles are also discussed.

The rest of this article is following as, in section 2, we briefly review some basic ideas such as CIVIFSs and their useful laws. In section 3, we explore a novel approach to CIVPFSs and their algebraic laws. In section 4, we explore certain Einstein operational laws by using the t-norm and t-conorm based on CIVPFSs. In section 5, we explore the CIVPFEWG, CIVPFEOWG operators, and utilized their special cases. In section 6, an MCDM technique is explored based on the elaborated operators by using the CIVPF information. To determine the consistency and reliability of the elaborated operators, we illustrated certain examples by using the explored principles. Finally, to determine the supremacy and dominance of the explored theories, the comparative analysis and graphical expressions of the developed principles are also discussed. In section 7, we discussed the conclusion of this manuscript.

\section{Preliminaries}

This study aims to briefly review some basic ideas such as CIVIFSs and their useful laws which are helpful for the elaborated approaches in the next study. Additionally, in the overall manuscript the symbols $\mathbb{X}_{u n i}$ expressed the fixed set and the value of truth grade (TG) and falsity grade (FG) are denoted by $\mathbb{T}_{\mathcal{F}_{c q}}=$ $\left[\mathbb{T}_{\mathcal{F}_{r p}}^{-}, \mathbb{T}_{\mathcal{F}_{r p}}^{+}\right] e^{i 2 \pi\left(\left[\mathbb{T}_{\mathcal{F}_{i p}}^{-} \mathbb{T}_{\mathcal{F}_{i p}}^{+}\right]\right)}$and $\mathbb{F}_{\mathcal{F}_{c q}}=\left[\mathbb{F}_{\mathcal{F}_{r p}}^{-}, \mathbb{F}_{\mathcal{F}_{r p}}^{+}\right] e^{i 2 \pi\left(\left[\mathbb{F}_{\mathcal{F}_{i p}}^{-} \mathbb{F}_{\mathcal{F}_{i p}}^{+}\right]\right)}$. By using the above information, the principle of CIVFS covers the $\mathrm{TG} \quad \mathbb{T}_{\mathcal{F}_{c q}}=\left[\mathbb{T}_{\mathcal{F}_{r p}}^{-}, \mathbb{T}_{\mathcal{F}_{r p}}^{+}\right] e^{i 2 \pi\left(\left[\mathbb{T}_{\mathcal{F}_{i p}}^{-} \mathbb{T}_{\mathcal{F}_{i p}}^{+}\right]\right)}$with a rule that is $\mathbb{T}_{\mathcal{F}_{r p}}^{+}, \mathbb{T}_{\mathcal{F}_{i p}}^{+}, \mathbb{T}_{\mathcal{F}_{r p}}^{-}, \mathbb{T}_{\mathcal{F}_{i p}}^{-} \in[0,1]$. But there were some troubles if a person faced such sort of information that covers the TG $\mathbb{T}_{\mathcal{F}_{c q}}=\left[\mathbb{T}_{\mathcal{F}_{r p}}^{-}, \mathbb{T}_{\mathcal{F}_{r p}}^{+}\right] e^{i 2 \pi\left(\left[\mathbb{T}_{\mathcal{F}_{i p}}^{-}, \mathbb{T}_{\mathcal{F}_{i p}}^{+}\right]\right)}$and FG $\mathbb{F}_{\mathcal{F}_{c q}}=\left[\mathbb{F}_{\mathcal{F}_{r p}}^{-}, \mathbb{F}_{\mathcal{F}_{r p}}^{+}\right] e^{i 2 \pi\left(\left[\mathbb{F}_{\mathcal{F}_{i p}}^{-}, \mathbb{F}_{\mathcal{F}_{i p}}^{+}\right]\right)}$, then the principle of CIVFS has been failed. To resolve these sorts of troubles, the principle of CIVIFS was developed by Garg and Rani (2019a) is discussed below.

Definition 1: (Garg and Rani, 2019a) An CIVIFS $\mathcal{F}_{c i}$ is stated by:

$$
\mathcal{F}_{c i}=\left\{\left(\mathbb{T}_{\mathcal{F}_{c i}}\left(\mathbb{x}_{e l}\right), \mathbb{F}_{\mathcal{F}_{c i}}\left(\mathbb{x}_{e l}\right)\right): \mathbb{x}_{e l} \in \mathbb{X}_{u n i}\right\}
$$

Where $\mathbb{T}_{\mathcal{F}_{c q}}=\left[\mathbb{T}_{\mathcal{F}_{r p}}^{-}, \mathbb{T}_{\mathcal{F}_{r p}}^{+}\right] e^{i 2 \pi\left(\left[\mathbb{T}_{\mathcal{F}_{i p}}^{-}, \mathbb{T}_{\mathcal{F}_{i p}}^{+}\right]\right)}$and $\mathbb{F}_{\mathcal{F}_{c q}}=\left[\mathbb{F}_{\mathcal{F}_{r p}}^{-}, \mathbb{F}_{\mathcal{F}_{r p}}^{+}\right] e^{i 2 \pi\left(\left[\mathbb{F}_{\mathcal{F}_{i p}}^{-}, \mathbb{F}_{\mathcal{F}_{i p}}^{+}\right]\right)}$with conditions that are $0 \leq \mathbb{T}_{\mathcal{F}_{r p}}^{+}\left(\mathbb{x}_{e l}\right)+\mathbb{F}_{\mathcal{F}_{r p}}^{+}\left(\mathbb{x}_{e l}\right) \leq 1,0 \leq \mathbb{T}_{\mathcal{F}_{i p}}^{+}\left(\mathbb{x}_{e l}\right)+\mathbb{F}_{\mathcal{F}_{i p}}^{+}\left(\mathbb{x}_{e l}\right) \leq 1$. The refusal grade (RG) is stated by $\pi_{\mathcal{F}_{c i}}\left(\mathbb{x}_{e l}\right)=\left[\pi_{\mathcal{F}_{r p}}^{-}\left(\mathbb{x}_{e l}\right), \pi_{\mathcal{F}_{r p}}^{+}\left(\mathbb{x}_{e l}\right)\right] e^{i 2 \pi\left(\left[\pi_{\overline{\mathcal{F}}_{i p}}\left(\mathbb{x}_{e l}\right), \pi_{\mathcal{F}_{i p}}^{+}\left(\mathbb{x}_{e l}\right)\right]\right)}=\left[1-\left(\mathbb{T}_{\mathcal{F}_{r p}}^{-}\left(\mathbb{x}_{e l}\right)+\mathbb{F}_{\mathcal{F}_{r p}}^{-}\left(\mathbb{x}_{e l}\right)\right), 1-\right.$ $\left.\left(\mathbb{T}_{\mathcal{F}_{r p}}^{+}\left(\mathbb{x}_{e l}\right)+\mathbb{F}_{\mathcal{F}_{r p}}^{+}\left(\mathbb{x}_{e l}\right)\right)\right] e^{i 2 \pi\left(\left[1-\left(\mathbb{T}_{\mathcal{F}_{i p}}^{-}\left(\mathbb{x}_{e l}\right)+\mathbb{F}_{\mathcal{F}_{i p}}^{-}\left(\mathbb{x}_{e l}\right)\right), 1-\left(\mathbb{T}_{\mathcal{F}_{i p}}^{+}\left(\mathbb{x}_{e l}\right)+\mathbb{F}_{\mathcal{F}_{i p}}^{+}\left(\mathbb{x}_{e l}\right)\right)\right]\right)}$. In the overall manuscript, the 


$$
\begin{aligned}
& \begin{array}{l}
\text { complex } \quad \text { interval-valued intuitionistic } \\
\mathcal{F}_{c i-j}=\left(\left[\mathbb{T}_{\mathcal{F}_{r p-j}}^{-}, \mathbb{T}_{\mathcal{F}_{r p-j}}^{+}\right] e^{i 2 \pi\left(\left[\mathbb{T}_{\mathcal{F}_{i p-j}}^{-} \mathbb{T}_{\mathcal{F}_{i p-j}}^{+}\right]\right)},\left[\mathbb{F}_{\mathcal{F}_{r p-j}}^{-}, \mathbb{F}_{\mathcal{F}_{r p-j}}^{+}\right] e^{i 2 \pi\left(\left[\mathbb{F}_{\mathcal{F}_{i p-j}}^{-} \mathbb{F}_{\mathcal{F}_{i p-j}}^{+}\right]\right)}\right), j=1,2, \ldots, \mathbb{m}_{l e}
\end{array} \\
& \text { Moreover, by using any two } \\
& \mathcal{F}_{c p-j}=\left(\left[\mathbb{T}_{\mathcal{F}_{r p-j}}^{-} \mathbb{T}_{\mathcal{F}_{r p-j}}^{+}\right] e^{i 2 \pi\left(\left[\mathbb{T}_{\mathcal{F}_{i p-j}}^{-}, \mathbb{T}_{\mathcal{F}_{i p-j}}^{+}\right]\right)},\left[\mathbb{F}_{\mathcal{F}_{r p-j}}^{-}, \mathbb{F}_{\mathcal{F}_{r p-j}}^{+}\right] e^{i 2 \pi\left(\left[\mathbb{F}_{\mathcal{F}_{i p-j}}^{-} \mathbb{F}_{\mathcal{F}_{i p-j}}^{+}\right]\right)}\right), j=1,2, \text { then }
\end{aligned}
$$

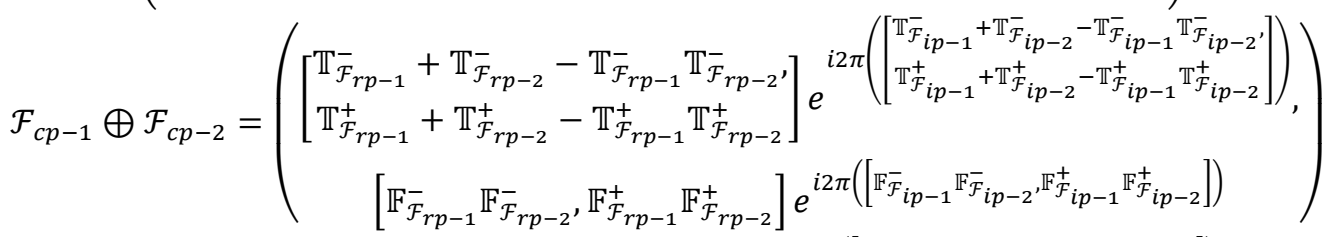

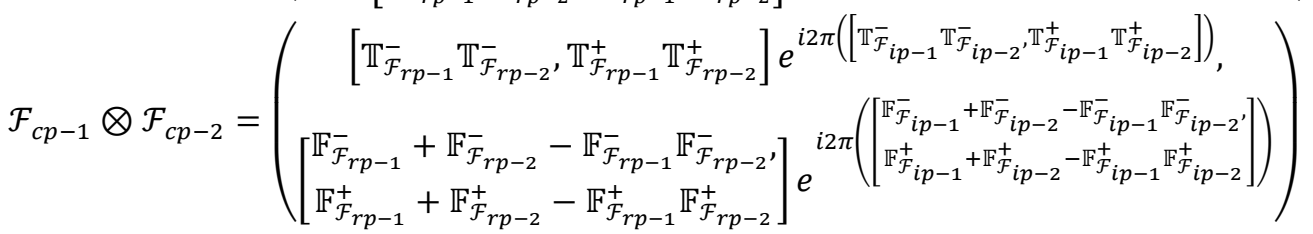

$$
\begin{aligned}
& \psi_{s c} \mathcal{F}_{c p-1}=
\end{aligned}
$$

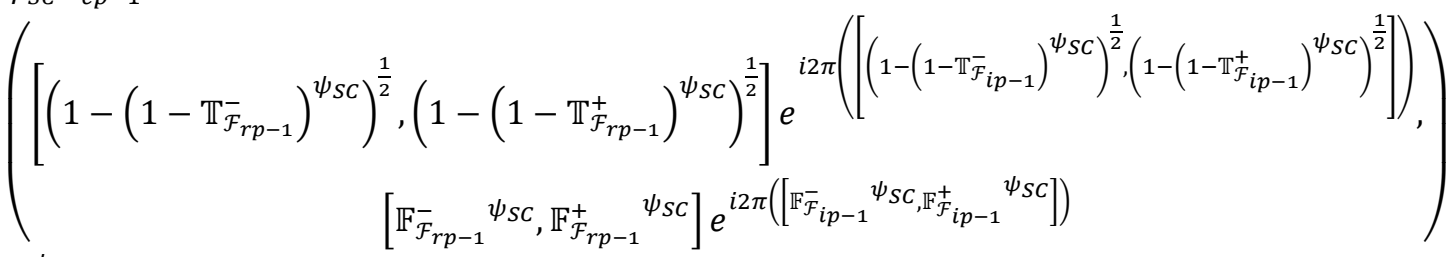

$$
\begin{aligned}
& \mathcal{F}_{c p-1}^{\psi_{s c}}=
\end{aligned}
$$

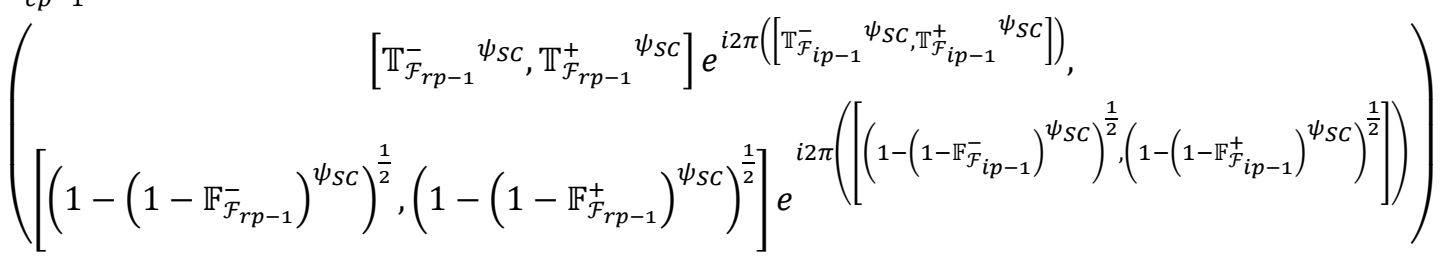

CIVIFNs

Moreover, to determine the interrelationships among any numbers of attributes, we briefly discussed the score function (SF) and accuracy function (AF), which are stated by:

$$
\begin{aligned}
& \mathbb{S}^{s f}\left(\mathcal{F}_{c p-1}\right)=\frac{1}{2}\left(\begin{array}{c}
\mathbb{T}_{\mathcal{F}_{r p-j}}^{-}+\mathbb{T}_{\mathcal{F}_{r p-j}}^{+}+\mathbb{T}_{\mathcal{F}_{i p-j}}^{-}+\mathbb{T}_{\mathcal{F}_{i p-j}}^{+}- \\
\mathbb{F}_{\mathcal{F}_{r p-j}}^{-}-\mathbb{F}_{\mathcal{F}_{r p-j}}^{+}-\mathbb{F}_{\mathcal{F}_{i p-j}}^{-}-\mathbb{F}_{\mathcal{F}_{i p-j}}^{+}
\end{array}\right), \mathbb{S}^{s f}\left(\mathcal{F}_{c p-1}\right) \in[-1,1] \\
& \mathbb{H}^{a f}\left(\mathcal{F}_{c p-1}\right)=\frac{1}{2}\left(\begin{array}{c}
\mathbb{T}_{\mathcal{F}_{r p-j}}^{-}+\mathbb{T}_{\mathcal{F}_{r p-j}}^{+}+\mathbb{T}_{\mathcal{F}_{i p-j}}^{-}+\mathbb{T}_{\mathcal{F}_{i p-j}}^{+}+ \\
\mathbb{F}_{\mathcal{F}_{r p-j}}^{-}+\mathbb{F}_{\mathcal{F}_{r p-j}}^{+}+\mathbb{F}_{\mathcal{F}_{i p-j}}^{-}+\mathbb{F}_{\mathcal{F}_{i p-j}}^{+}
\end{array}\right), \mathbb{H}^{a f}\left(\mathcal{F}_{c p-1}\right) \in[0,1] \\
& \text { By } \quad \text { asing }
\end{aligned}
$$

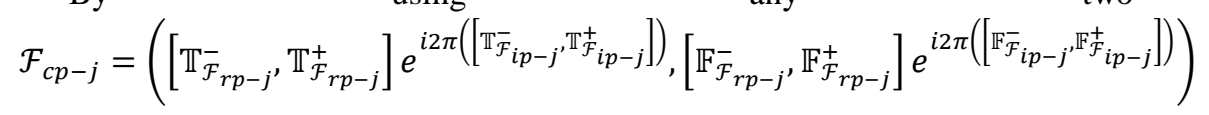

$$
\text { , } j=1,2 \text {, then }
$$

1. If $\mathbb{S}^{s f}\left(\mathcal{F}_{c p-1}\right)>\mathbb{S}^{s f}\left(\mathcal{F}_{c p-2}\right) \Rightarrow \mathcal{F}_{c p-1}>\mathcal{F}_{c p-2}$;

2. If $\mathbb{S}^{s f}\left(\mathcal{F}_{c p-1}\right)<\mathbb{S}^{s f}\left(\mathcal{F}_{c p-2}\right) \Rightarrow \mathcal{F}_{c p-1}<\mathcal{F}_{c p-2}$;

3. If $\mathbb{S}^{s f}\left(\mathcal{F}_{c p-1}\right)=\mathbb{S}^{s f}\left(\mathcal{F}_{c p-2}\right) \Rightarrow$

1) If $\mathbb{H}^{a f}\left(\mathcal{F}_{c p-1}\right)>\mathbb{H}^{a f}\left(\mathcal{F}_{c p-2}\right) \Rightarrow \mathcal{F}_{c p-1}>\mathcal{F}_{c p-2}$;

2) If $\mathbb{H}^{a f}\left(\mathcal{F}_{c p-1}\right)<\mathbb{H}^{a f}\left(\mathcal{F}_{c p-2}\right) \Rightarrow \mathcal{F}_{c p-1}<\mathcal{F}_{c p-2}$;

3) If $\mathbb{H}^{a f}\left(\mathcal{F}_{c p-1}\right)=\mathbb{H}^{a f}\left(\mathcal{F}_{c p-2}\right) \Rightarrow \mathcal{F}_{c p-1}=\mathcal{F}_{c p-2}$.

\section{Complex Interval-valued Pythagorean Fuzzy sets}

But there were some troubles if a person faced such sort of information which covers the $\mathrm{TG} \mathbb{T}_{\mathcal{F}_{c i}}=$

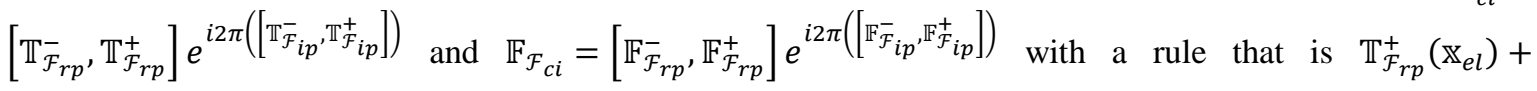


$\mathbb{F}_{\mathcal{F}_{r p}}^{+}\left(\mathbb{X}_{e l}\right), \mathbb{T}_{\mathcal{F}_{i p}}^{+}\left(\mathbb{X}_{e l}\right)+\mathbb{F}_{\mathcal{F}_{i p}}^{+}\left(\mathbb{x}_{e l}\right)>1$, then the principle of CIVIFS has been failed. For instance, if we gave $[0.5,0.6] e^{i 2 \pi([0.3,0.5])}$ for TG and $[0.1,0.5] e^{i 2 \pi([0.3,0.6])}$ for FG, then by using the conditions of CIVIFS is failed i.e., $0.6+0.5=1.1>1,0.5+0.6=1.1>1$. To resolve these sorts of troubles, the principle of CIVPFS is explored in this study. By using the theory of CIVPFS, certain laws are also elaborated.

Definition 2: A CIVPFS $\mathcal{F}_{c p}$ is stated by:

$\mathcal{F}_{c p}=\left\{\left(\mathbb{T}_{\mathcal{F}_{c p}}\left(\mathbb{x}_{e l}\right), \mathbb{F}_{\mathcal{F}_{c p}}\left(\mathbb{x}_{e l}\right)\right): \mathbb{x}_{e l} \in \mathbb{X}_{\text {uni }}\right\}$

Where $\mathbb{T}_{\mathcal{F}_{c p}}=\left[\mathbb{T}_{\mathcal{F}_{r p}}^{-}, \mathbb{T}_{\mathcal{F}_{r p}}^{+}\right] e^{i 2 \pi\left(\left[\mathbb{T}_{\mathcal{F}_{i p}}^{-}, \mathbb{T}_{\mathcal{F}_{i p}}^{+}\right]\right)}$and $\mathbb{F}_{\mathcal{F}_{c p}}=\left[\mathbb{F}_{\mathcal{F}_{r p}}^{-}, \mathbb{F}_{\mathcal{F}_{r p}}^{+}\right] e^{i 2 \pi\left(\left[\mathbb{F}_{\mathcal{F}_{i p}}^{-}, \mathbb{F}_{\mathcal{F}_{i p}}^{+}\right]\right)}$with certain rules that are., $0 \leq \mathbb{T}_{\mathcal{F}_{r p}}^{+}{ }^{2}\left(\mathbb{x}_{e l}\right)+\mathbb{F}_{\mathcal{F}_{r p}}^{+}{ }^{2}\left(\mathbb{x}_{e l}\right) \leq 1,0 \leq \mathbb{T}_{\mathcal{F}_{i p}}^{+}{ }^{2}\left(\mathbb{x}_{e l}\right)+\mathbb{F}_{\mathcal{F}_{i p}}^{+}{ }^{2}\left(\mathbb{x}_{e l}\right) \leq 1$. The RG is stated by $\pi_{\mathcal{F}_{p f s}}\left(\mathbb{x}_{e l}\right)=$ $\left[\pi_{\mathcal{F}_{r p}}^{-}\left(\mathbb{x}_{e l}\right), \pi_{\mathcal{F}_{r p}}^{+}\left(\mathbb{x}_{e l}\right)\right] e^{i 2 \pi\left(\left[\pi_{\mathcal{F}_{i p}}^{-}\left(\mathbb{x}_{e l}\right), \pi_{\mathcal{F}_{i p}}^{+}\left(\mathbb{x}_{e l}\right)\right]\right)}=\left[\left(1-\left(\mathbb{T}_{\mathcal{F}_{r p}}^{-}{ }^{2}\left(\mathbb{x}_{e l}\right)+\mathbb{F}_{\mathcal{F}_{r p}}^{-}{ }^{2}\left(\mathbb{x}_{e l}\right)\right)\right)^{\frac{1}{2}},\left(1-\left(\mathbb{T}_{\mathcal{F}_{r p}}^{+}{ }^{2}\left(\mathbb{x}_{e l}\right)+\right.\right.\right.$ $\left.\left.\left.\mathbb{F}_{\mathcal{F}_{r p}}^{+}{ }^{2}\left(\mathbb{x}_{e l}\right)\right)\right)^{\frac{1}{2}}\right] e^{i 2 \pi\left(\left[\left(1-\left(\mathbb{T}_{\mathcal{F}_{i p}}^{-}{ }^{2}\left(\mathbb{x}_{e l}\right)+\mathbb{F}_{\mathcal{F}_{i p}}{ }^{2}\left(\mathbb{x}_{e l}\right)\right)\right)^{\frac{1}{2}},\left(1-\left(\mathbb{T}_{\mathcal{F}_{i p}}^{+}{ }^{2}\left(\mathrm{x}_{e l}\right)+\mathbb{F}_{\mathcal{F}}^{+}{ }^{2}{ }^{2}\left(\mathbb{x}_{e l}\right)\right)\right)^{\frac{1}{2}}\right]\right)}$. In the overall manuscript, the complex interval-valued Pythagorean fuzzy numbers (CIVPFNs) are stated by $\mathcal{F}_{c p-j}=\left(\left[\mathbb{T}_{\mathcal{F}_{r p-j}}^{-}, \mathbb{T}_{\mathcal{F}_{r p-j}}^{+}\right] e^{i 2 \pi\left(\left[\mathbb{T}_{\mathcal{F}_{i p-j}}^{-}, \mathbb{T}_{\mathcal{F}_{i p-j}}^{+}\right]\right)},\left[\mathbb{F}_{\mathcal{F}_{r p-j}}^{-}, \mathbb{F}_{\mathcal{F}_{r p-j}}^{+}\right] e^{i 2 \pi\left(\left[\mathbb{F}_{\mathcal{F}_{i p-j}}, \mathbb{F}_{\mathcal{F}_{i p-j}}^{+}\right]\right)}\right), j=1,2, \ldots, \mathbb{m}_{l e}$ by using any two

CPFNs $\mathcal{F}_{c p-j}=\left(\left[\mathbb{T}_{\mathcal{F}_{r p-j}}^{-}, \mathbb{T}_{\mathcal{F}_{r p-j}}^{+}\right] e^{i 2 \pi\left(\left[\mathbb{T}_{\mathcal{F}_{i p-j}}^{-} \mathbb{T}_{\mathcal{F}_{i p-j}}^{+}\right]\right)},\left[\mathbb{F}_{\mathcal{F}_{r p-j}}^{-}, \mathbb{F}_{\mathcal{F}_{r p-j}}^{+}\right] e^{i 2 \pi\left(\left[\mathbb{F}_{\mathcal{F}_{i p-j}}^{-} \mathbb{F}_{\mathcal{F}_{i p-j}}^{+}\right]\right)}\right), j=1,2$, then $\mathcal{F}_{c p-1} \oplus \mathcal{F}_{c p-2}=$

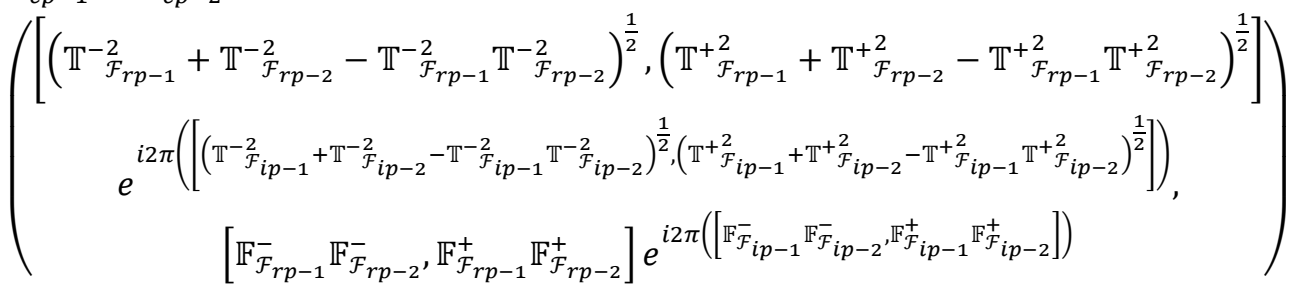

$\mathcal{F}_{c p-1} \otimes \mathcal{F}_{c p-2}=$

$$
\begin{aligned}
& \left(\begin{array}{c}
{\left[\mathbb{T}_{\mathcal{F}_{r p-1}}^{-} \mathbb{T}_{\mathcal{F}_{r p-2}}^{-}, \mathbb{T}_{\mathcal{F}_{r p-1}}^{+} \mathbb{T}_{\mathcal{F}_{r p-2}}^{+}\right] e^{i 2 \pi\left(\left[\mathbb{T}_{\mathcal{F}_{i p-1}}^{-} \mathbb{T}_{\mathcal{F}_{i p-2}}^{-} \mathbb{T}_{\mathcal{F}_{i p-1}}^{+} \mathbb{T}_{\mathcal{F}_{i p-2}}^{+}\right]\right)},} \\
{\left[\left(\mathbb{F}_{\mathcal{F}_{r p-1}}^{-2}+\mathbb{F}_{\mathcal{F}_{r p-2}}^{-2}-\mathbb{F}_{\mathcal{F}_{r p-1}}^{-2} \mathbb{F}_{\mathcal{F}_{r p-2}}^{-2}\right)^{\frac{1}{2}},\left(\mathbb{F}_{\mathcal{F}_{r p-1}}^{+2}+\mathbb{F}_{\mathcal{F}_{r p-2}}^{+2}-\mathbb{F}_{\mathcal{F}_{r p-1}}^{+2} \mathbb{F}_{\mathcal{F}_{r p-2}}^{+2}\right)^{\frac{1}{2}}\right]} \\
e^{i 2 \pi\left(\left[\left(\mathbb{F}_{\mathcal{F}_{i p-1}}^{-2}+\mathbb{F}^{-2} \mathcal{F}_{i p-2}-\mathbb{F}_{\mathcal{F}_{i p-1}}^{-2} \mathbb{F}_{\mathcal{F}_{i p-2}}^{-2}\right)^{\frac{1}{2}},\left(\mathbb{F}^{+}{ }_{\mathcal{F}_{i p-1}}^{2}+\mathbb{F}_{\mathcal{F}_{i p-2}}^{+2}-\mathbb{F}_{\mathcal{F}_{i p-1}}^{+2} \mathbb{F}_{\mathcal{F}_{i p-2}}^{+2}\right)^{\frac{1}{2}}\right]\right)}
\end{array}\right)
\end{aligned}
$$

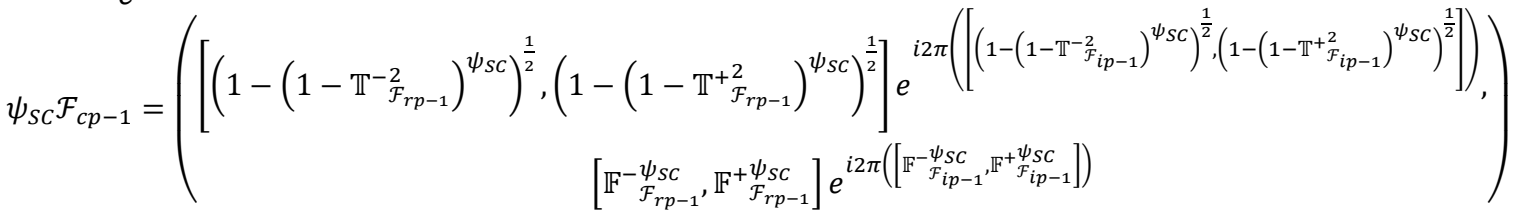

$$
\begin{aligned}
& \mathcal{F}_{c p-1}^{\psi_{S C}}=
\end{aligned}
$$

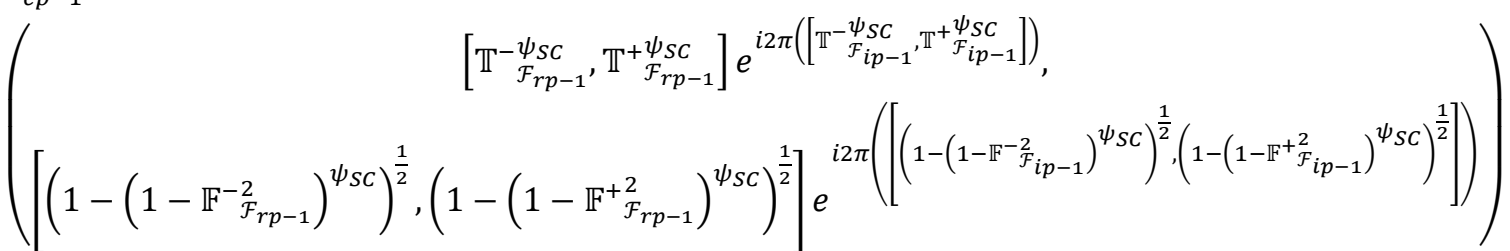

(12)

If we choose the value of $2=1$, then the information in Eq. (9) to Eq. (12) is reduced for CIVIFSs. Moreover, to determine the interrelationships among any numbers of attributes, we briefly discussed the score function (SF) and accuracy function (AF), which are stated by: 


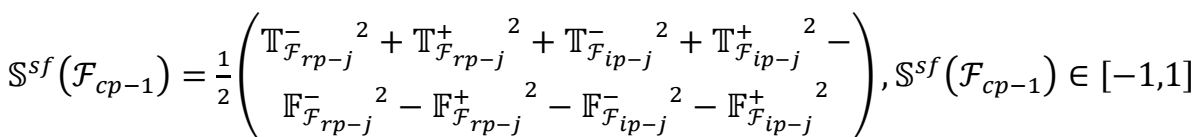

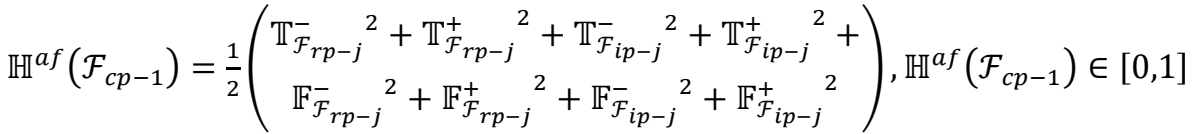

By using

any

two

CIVPFNs

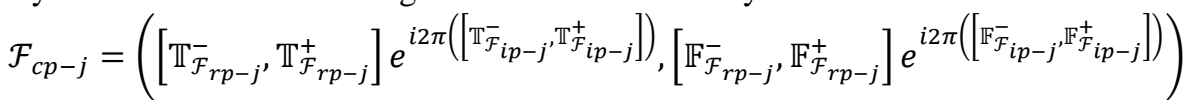

, $j=1,2$, then

1. If $\mathbb{S}^{s f}\left(\mathcal{F}_{c p-1}\right)>\mathbb{S}^{s f}\left(\mathcal{F}_{c p-2}\right) \Rightarrow \mathcal{F}_{c p-1}>\mathcal{F}_{c p-2}$;

2. If $\mathbb{S}^{s f}\left(\mathcal{F}_{c p-1}\right)<\mathbb{S}^{s f}\left(\mathcal{F}_{c p-2}\right) \Rightarrow \mathcal{F}_{c p-1}<\mathcal{F}_{c p-2}$;

3. If $\mathbb{S}^{s f}\left(\mathcal{F}_{c p-1}\right)=\mathbb{S}^{s f}\left(\mathcal{F}_{c p-2}\right) \Rightarrow$

1) If $\mathbb{H}^{a f}\left(\mathcal{F}_{c p-1}\right)>\mathbb{H}^{a f}\left(\mathcal{F}_{c p-2}\right) \Rightarrow \mathcal{F}_{c p-1}>\mathcal{F}_{c p-2}$;

2) If $\mathbb{H}^{a f}\left(\mathcal{F}_{c p-1}\right)<\mathbb{H}^{a f}\left(\mathcal{F}_{c p-2}\right) \Rightarrow \mathcal{F}_{c p-1}<\mathcal{F}_{c p-2}$;

3) If $\mathbb{H}^{a f}\left(\mathcal{F}_{c p-1}\right)=\mathbb{H}^{a f}\left(\mathcal{F}_{c p-2}\right) \Rightarrow \mathcal{F}_{c p-1}=\mathcal{F}_{c p-2}$.

\section{Einstein Operational laws for CIVPFNs}

Certain scholars have elaborated numerous t-norm and t-conorm, but Einstein t-norm and t-conorm have received extensive attraction from researchers. The main idea of Einstein t-norm and t-conorm are elaborated below.

$S(a, b)=\left(\frac{a^{2}+b^{2}}{1+a^{2} b^{2}}\right)^{\frac{1}{2}}, T(a, b)=\frac{a b}{\left(1+\left(1-a^{2}\right)\left(1-b^{2}\right)\right)^{\frac{1}{2}}}$

By using these laws, Wang and Liu (2020) were utilized the Einstein operational laws in the environment of intuitionistic fuzzy geometric aggregation operators. The principle of Einstein laws is also utilized in the environment of intuitionistic fuzzy information aggregation was elaborated by Wang and Liu (2012). Keeping the supremacy of the elaborated laws, Garg (2017) explored the principle of generalized Pythagorean fuzzy geometric aggregation operators based on Einstein laws. No one explored utilized the principle of Einstein laws in the environment of complex interval-valued intuitionistic and complex intervalvalued Pythagorean fuzzy sets. To improve the quality of research works, in this study, we explore some operational laws by using the Einstein laws and discussed their important results.

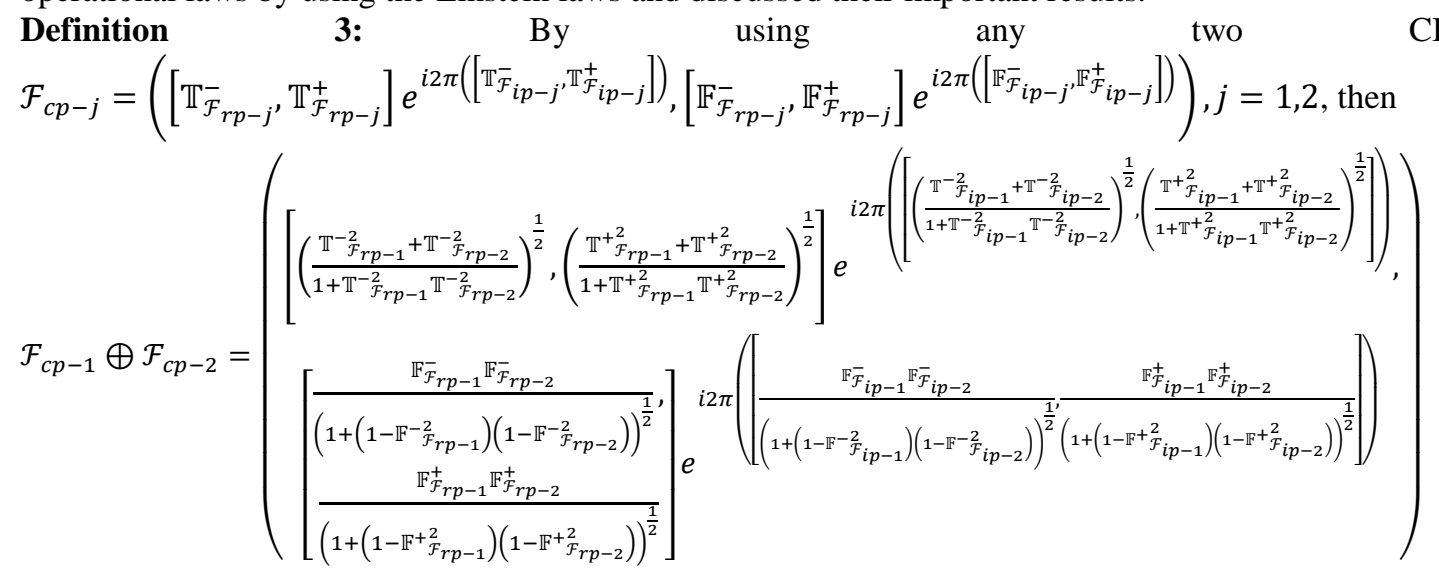




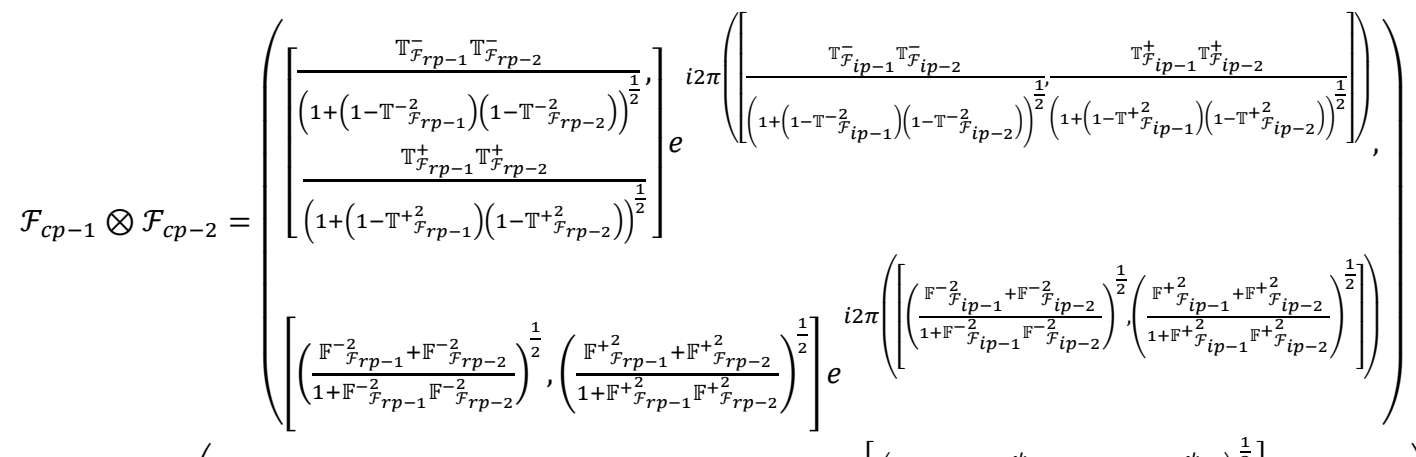

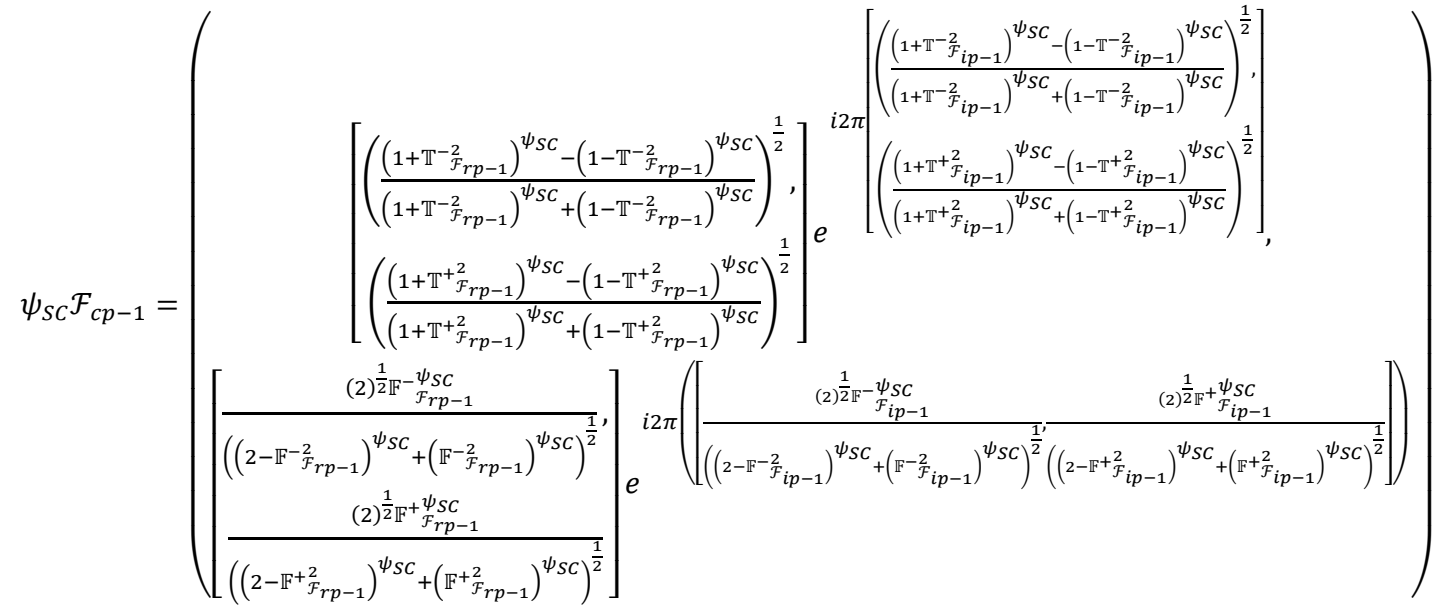

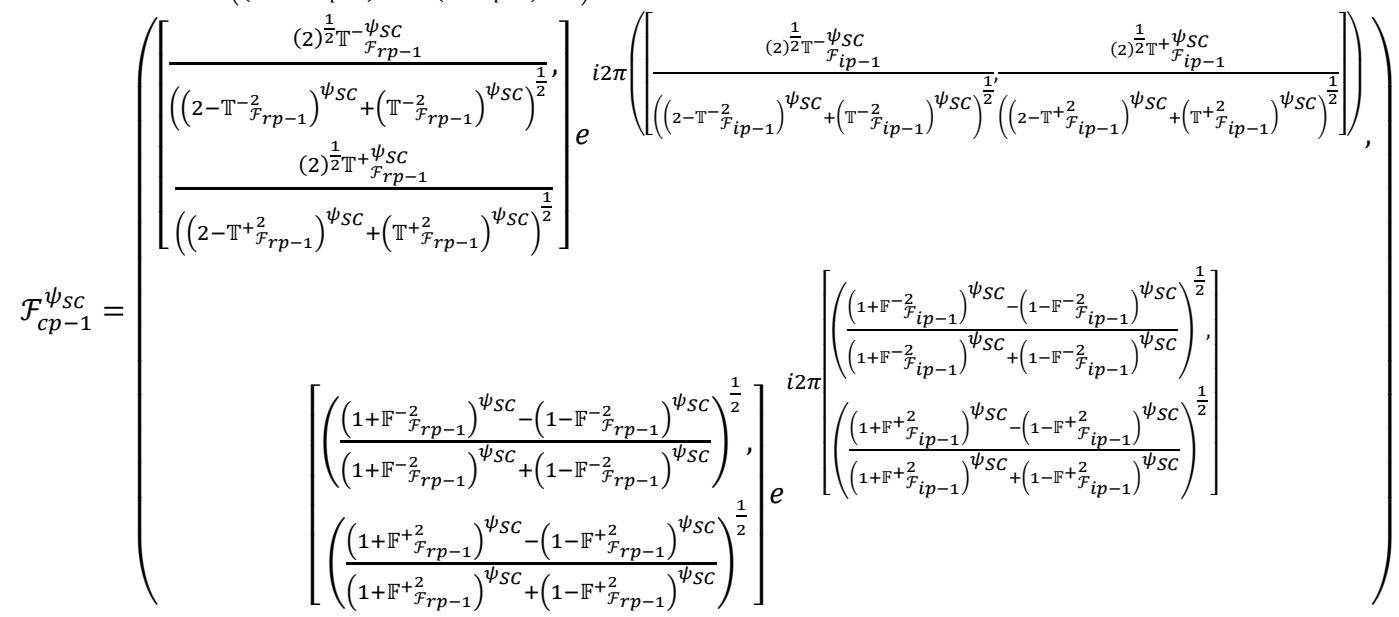

$\begin{array}{llllll}\text { Theorem 1: } & \text { By } & \text { using }\end{array}$ $\mathcal{F}_{c p-j}=\left(\left[\mathbb{T}_{\mathcal{F}_{r p-j}}^{-}, \mathbb{T}_{\mathcal{F}_{r p-j}}^{+}\right] e^{i 2 \pi\left(\left[\mathbb{T}_{\mathcal{F}_{i p-j}}^{-}, \mathbb{T}_{\mathcal{F}_{i p-j}}^{+}\right]\right)},\left[\mathbb{F}_{\mathcal{F}_{r p-j}}^{-}, \mathbb{F}_{\mathcal{F}_{r p-j}}^{+}\right] e^{i 2 \pi\left(\left[\mathbb{F}_{\mathcal{F}_{i p-j}}^{-}, \mathbb{F}_{\mathcal{F}_{i p-j}}^{+}\right]\right)}\right), j=1,2$, with $\psi_{S C-j}$, then

1. $\quad \mathcal{F}_{c p-1} \otimes \mathcal{F}_{c p-2}=\mathcal{F}_{c p-2} \otimes \mathcal{F}_{c p-1}$

2. $\mathcal{F}_{c p-1} \oplus \mathcal{F}_{c p-2}=\mathcal{F}_{c p-2} \oplus \mathcal{F}_{c p-1}$;

3. $\left(\mathcal{F}_{c p-1} \otimes \mathcal{F}_{c p-2}\right)^{\psi_{S C}}=\mathcal{F}_{c p-1}^{\psi_{S C}} \otimes \mathcal{F}_{c p-2}^{\psi_{S C}}$;

4. $\quad \psi_{S C}\left(\mathcal{F}_{c p-1} \oplus \mathcal{F}_{c p-2}\right)=\psi_{S C} \mathcal{F}_{c p-1} \oplus \psi_{S C} \mathcal{F}_{c p-2}$

5. $\mathcal{F}_{c p-1}^{\psi_{S C-1}} \oplus \mathcal{F}_{c p-1}^{\psi_{S C-2}}=\mathcal{F}_{c p-1}^{\psi_{S C-1}+\psi_{S C-2}}$;

6. $\quad \psi_{S C-1} \mathcal{F}_{c p-1} \otimes \psi_{S C-2} \mathcal{F}_{c p-1}=\left(\psi_{S C-1}+\psi_{S C-2}\right) \mathcal{F}_{c p-1}$;

7. $\left(\mathcal{F}_{c p-1}^{\psi_{S C-1}}\right)^{\psi_{S C-2}}=\mathcal{F}_{c p-1}^{\left(\psi_{S C-1} * \psi_{S C-2}\right)}$;

8. $\psi_{S C-1}\left(\psi_{S C-2} \mathcal{F}_{c p-1}\right)=\left(\psi_{S C-1} * \psi_{S C-2}\right) \mathcal{F}_{c p-1}$. 
$\begin{array}{llllll}\text { Proof: } & \text { By } & \text { using } & \text { two } & \text { CIVPFNs }\end{array}$ $\mathcal{F}_{c p-j}=\left(\left[\mathbb{T}_{\mathcal{F}_{r p-j}}^{-}, \mathbb{T}_{\mathcal{F}_{r p-j}}^{+}\right] e^{i 2 \pi\left(\left[\mathbb{T}_{\mathcal{F}_{i p-j}}^{-} \mathbb{T}^{+}{ }_{i p-j}\right]\right)},\left[\mathbb{F}_{\mathcal{F}_{r p-j}}^{-}, \mathbb{F}_{\mathcal{F}_{r p-j}}^{+}\right] e^{i 2 \pi\left(\left[\mathbb{F}_{\overline{\mathcal{F}}_{i p-j}}^{-} \mathbb{F}_{\mathcal{F}_{i p-j}}^{+}\right]\right)}\right), j=1,2$, with $\psi_{S C-j}$, we will prove that the (1), (3), and (5), other is similar.

1. By using the Eq. (17), such that

$$
\begin{aligned}
& \mathcal{F}_{c p-1} \otimes \mathcal{F}_{c p-2}
\end{aligned}
$$

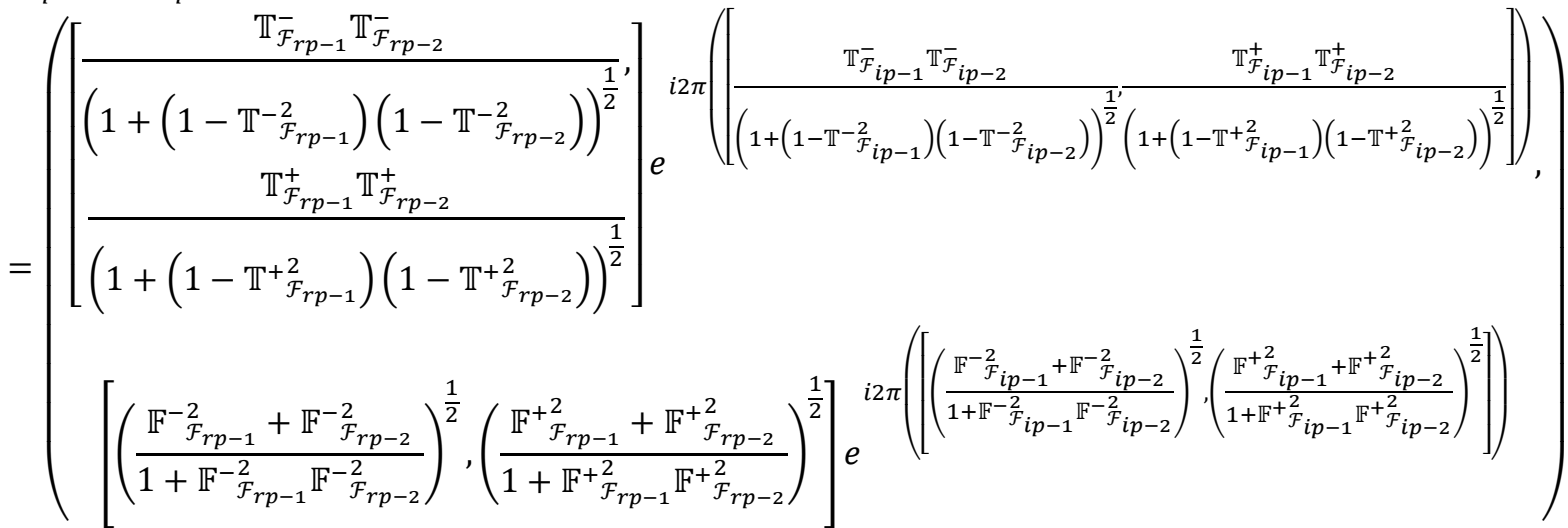

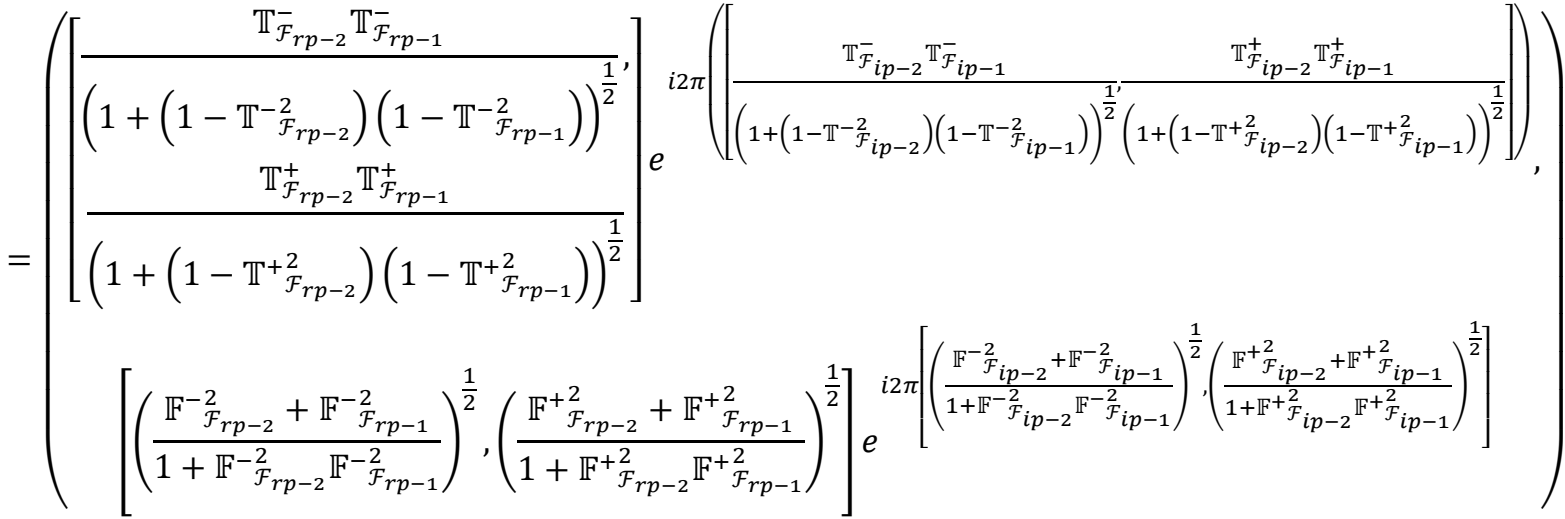

$$
\begin{aligned}
& =\mathcal{F}_{c p-2} \otimes \mathcal{F}_{c p-1}
\end{aligned}
$$

2. Straightforward.

3. By using Eq. (17), such that

$\mathcal{F}_{c p-1} \otimes \mathcal{F}_{c p-2}$

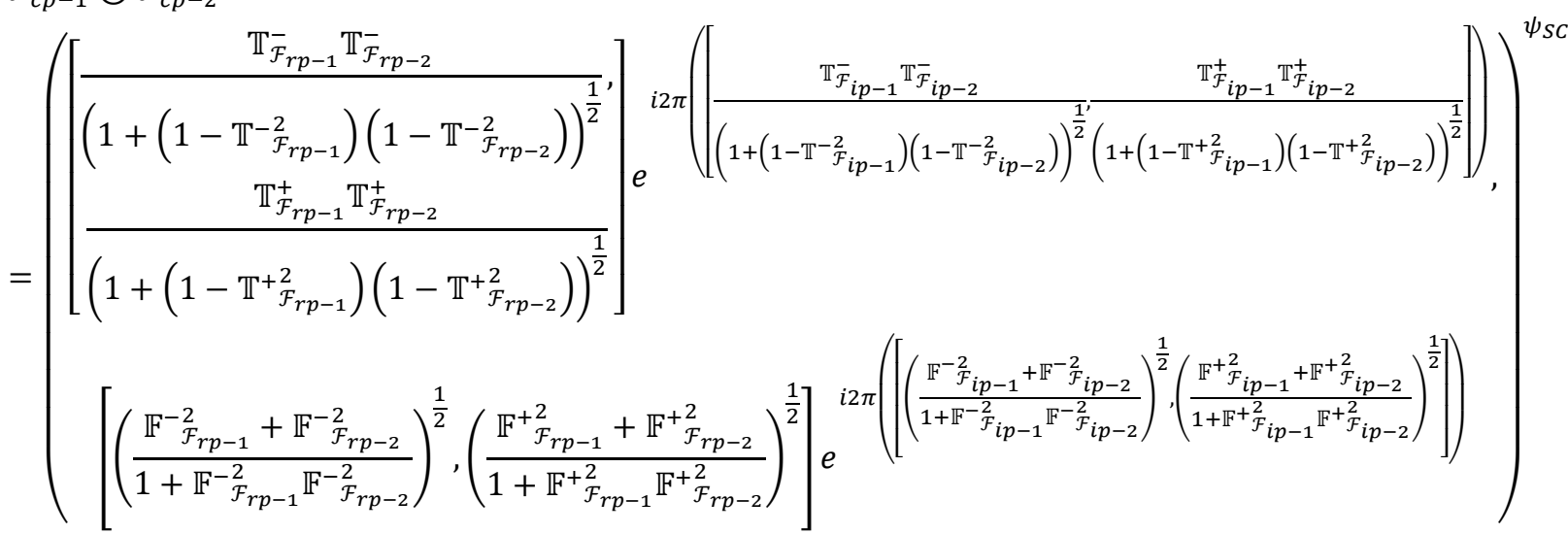




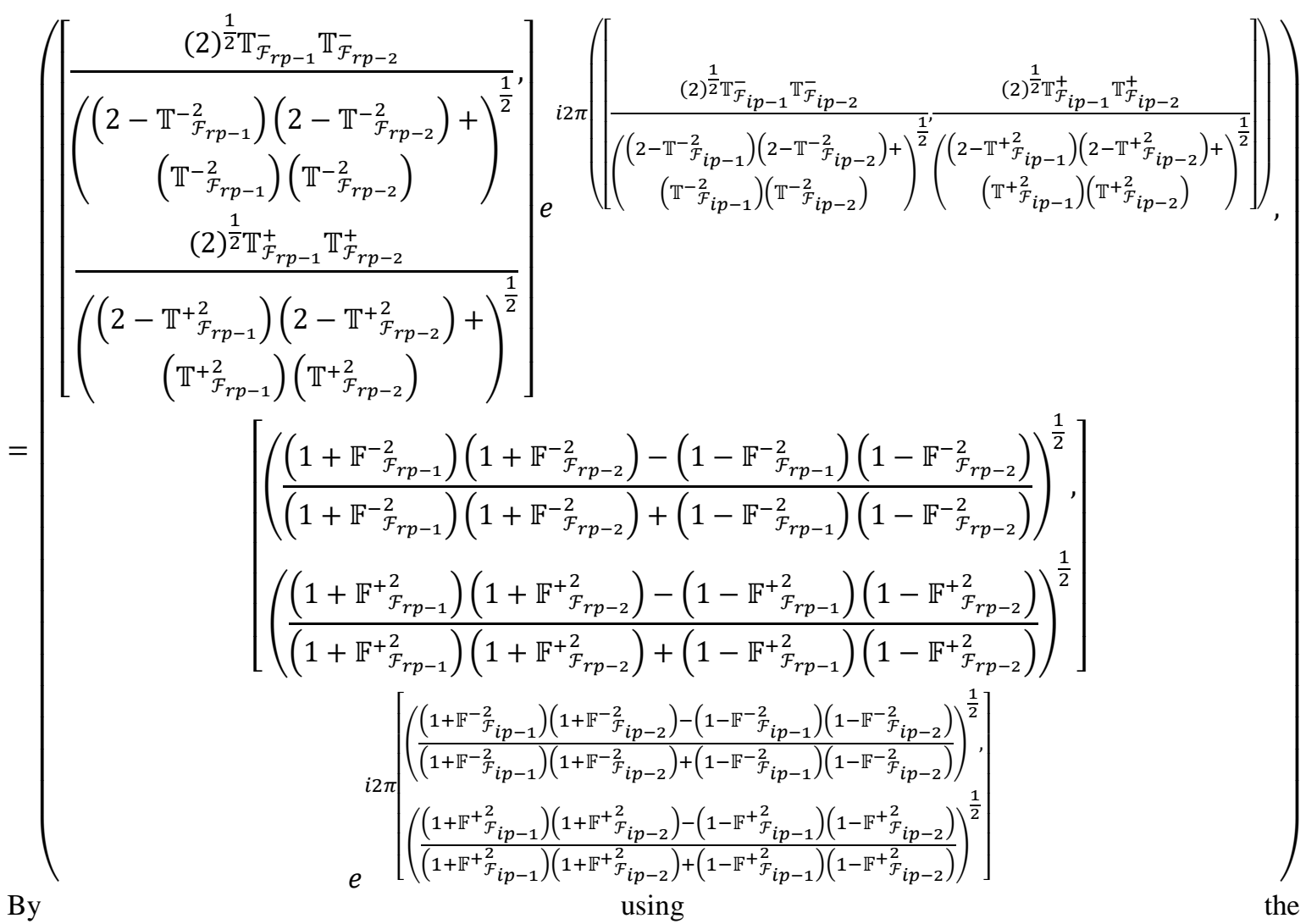

$a^{-}=\left(1+\mathbb{F}_{\mathcal{F}_{r p-1}}^{-2}\right)\left(1+\mathbb{F}_{\mathcal{F}_{r p-2}}^{-2}\right), b^{-}=\left(1-\mathbb{F}_{\mathcal{F}_{r p-1}}^{-2}\right)\left(1-\mathbb{F}_{\mathcal{F}_{r p-2}}^{-2}\right), c^{-}=\mathbb{T}_{\mathcal{F}_{r p-1}}^{-} \mathbb{T}_{\mathcal{F}_{r p-2}}^{-}, d^{-}=$ $\left(2-\mathbb{T}_{\mathcal{F}_{r p-1}}^{-2}\right)\left(2-\mathbb{T}_{\mathcal{F}_{r p-2}}^{-2}\right), a^{+}=\left(1+\mathbb{F}_{\mathcal{F}_{r p-1}}^{+2}\right)\left(1+\mathbb{F}_{\mathcal{F}_{r p-2}}^{+2}\right), b^{+}=\left(1-\mathbb{F}_{\mathcal{F}_{r p-1}}^{+2}\right)(1-$ $\left.\mathbb{F}_{\mathcal{F}_{r p-2}}^{+2}\right), c^{+}=\mathbb{T}_{\mathcal{F}_{r p-1}}^{+} \mathbb{T}_{\mathcal{F}_{r p-2}}^{+}, d^{+}=\left(2-\mathbb{T}_{\mathcal{F}_{r p-1}}^{+2}\right)\left(2-\mathbb{T}_{\mathcal{F}_{r p-2}}^{+2}\right) \quad$ and $\quad a^{-\prime}=\left(1+\mathbb{F}_{\mathcal{F}_{i p-1}}^{-2}\right)(1+$ $\left.\mathbb{F}_{\mathcal{F}_{i p-2}}^{-2}\right), b^{-\prime}=\left(1-\mathbb{F}_{\mathcal{F}_{i p-1}}^{-2}\right)\left(1-\mathbb{F}_{\mathcal{F}_{i p-2}}^{-2}\right), c^{-\prime}=\mathbb{T}_{\mathcal{F}_{i p-1}}^{-} \mathbb{T}_{\mathcal{F}_{i p-2}}^{-} d^{-^{\prime}}=\left(2-\mathbb{T}_{\mathcal{F}_{i p-1}}^{-2}\right)(2-$ $\left.\mathbb{T}_{\mathcal{F}_{i p-2}}^{-2}\right), a^{+^{\prime}}=\left(1+\mathbb{F}_{\mathcal{F}_{i p-1}}^{+2}\right)\left(1+\mathbb{F}_{\mathcal{F}_{i p-2}}^{+2}\right), b^{+^{\prime}}=\left(1-\mathbb{F}_{\mathcal{F}_{i p-1}}^{+2}\right)\left(1-\mathbb{F}_{\mathcal{F}_{i p-2}}^{+2}\right),{c^{+}}^{+^{\prime}}=$ $\mathbb{T}_{\mathcal{F}_{i p-1}}^{+} \mathbb{T}_{\mathcal{F}_{i p-2}}^{+}, d^{+^{\prime}}=\left(2-\mathbb{T}_{\mathcal{F}_{i p-1}}^{+2}\right)\left(2-\mathbb{T}_{\mathcal{F}_{i p-2}}^{+2}\right)$, then

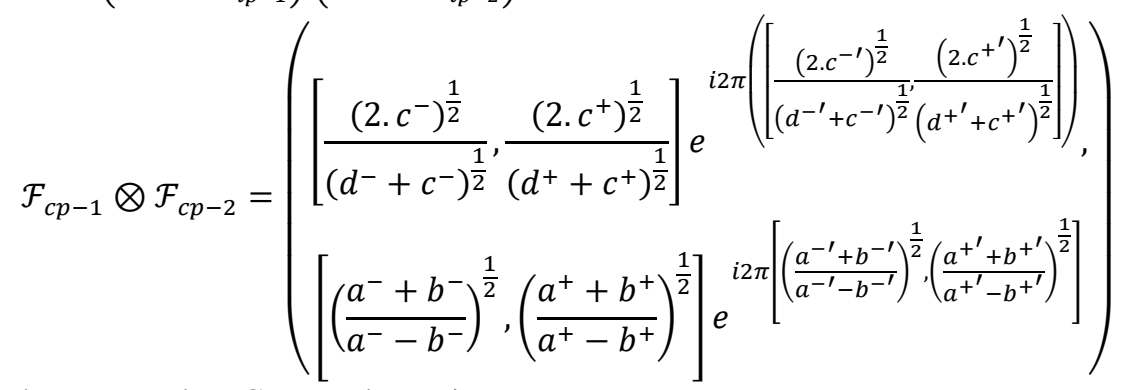

Then by using the ETN and ETCN, we determine

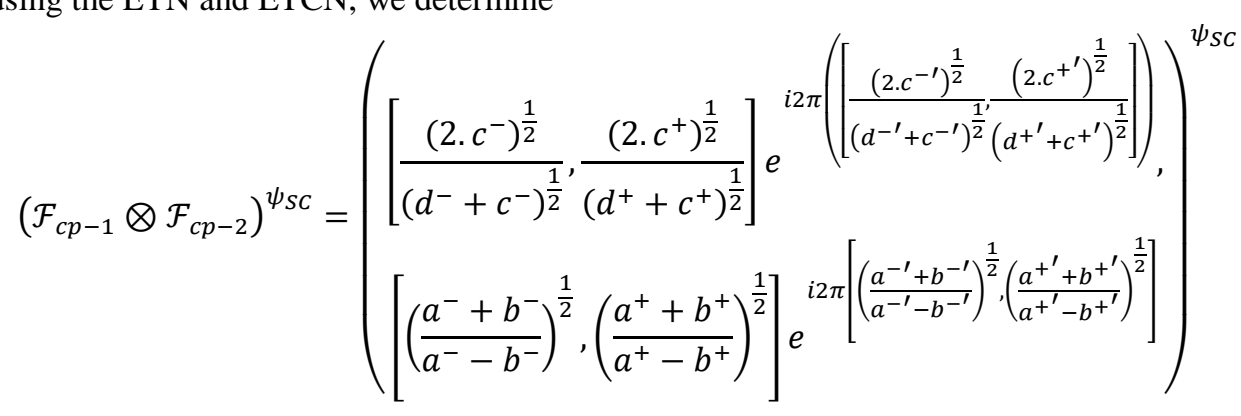


$114 \square$

ISSN 2683-5894

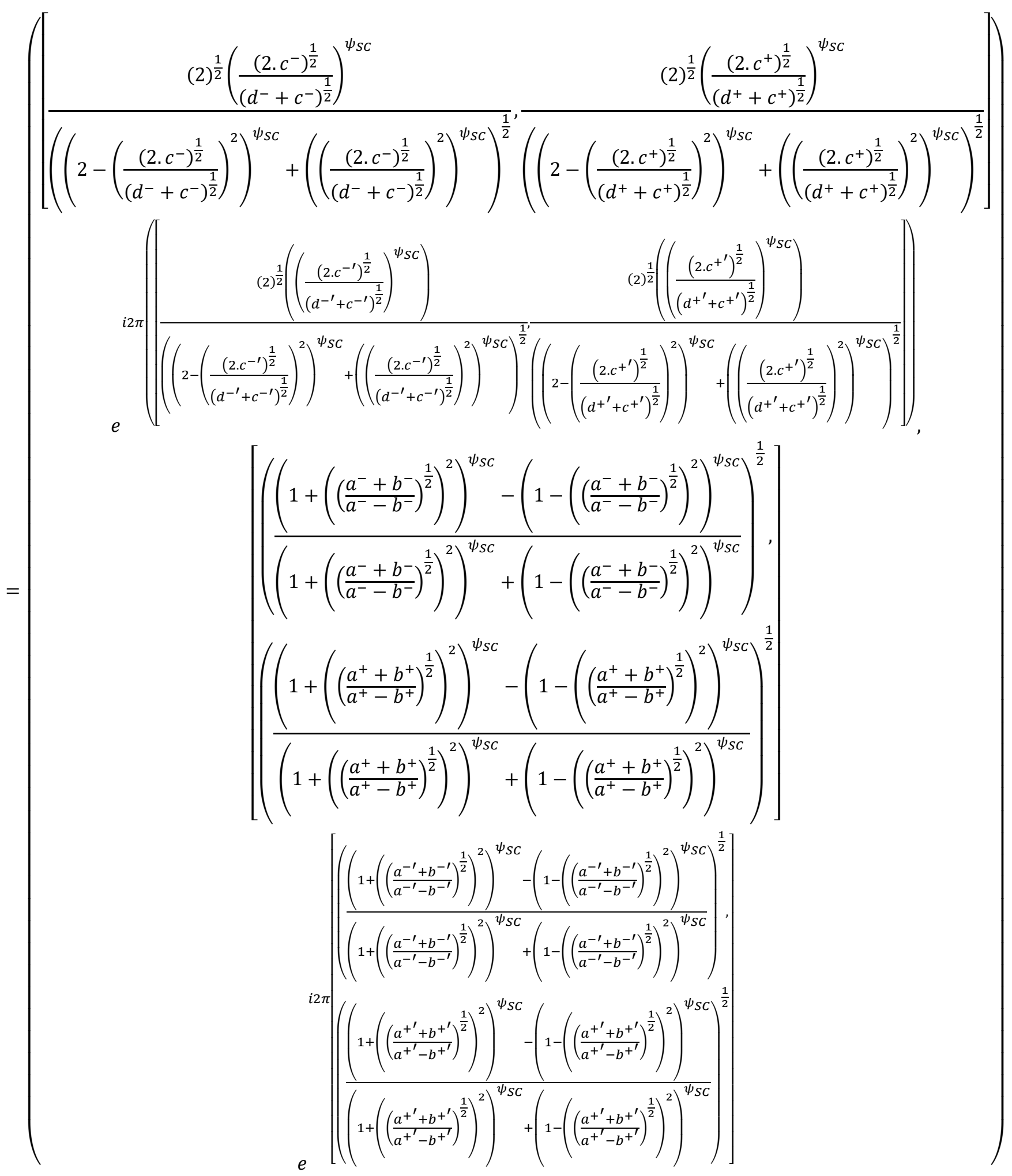

Reports in Mechanical Engineering, Vol. 2, No. 1, 2021: 105 - 134 


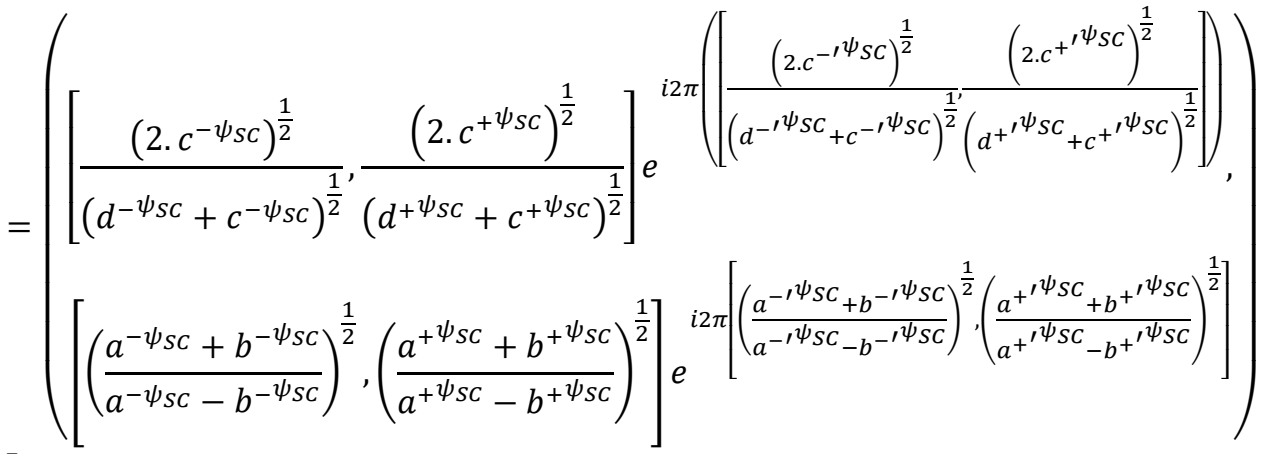

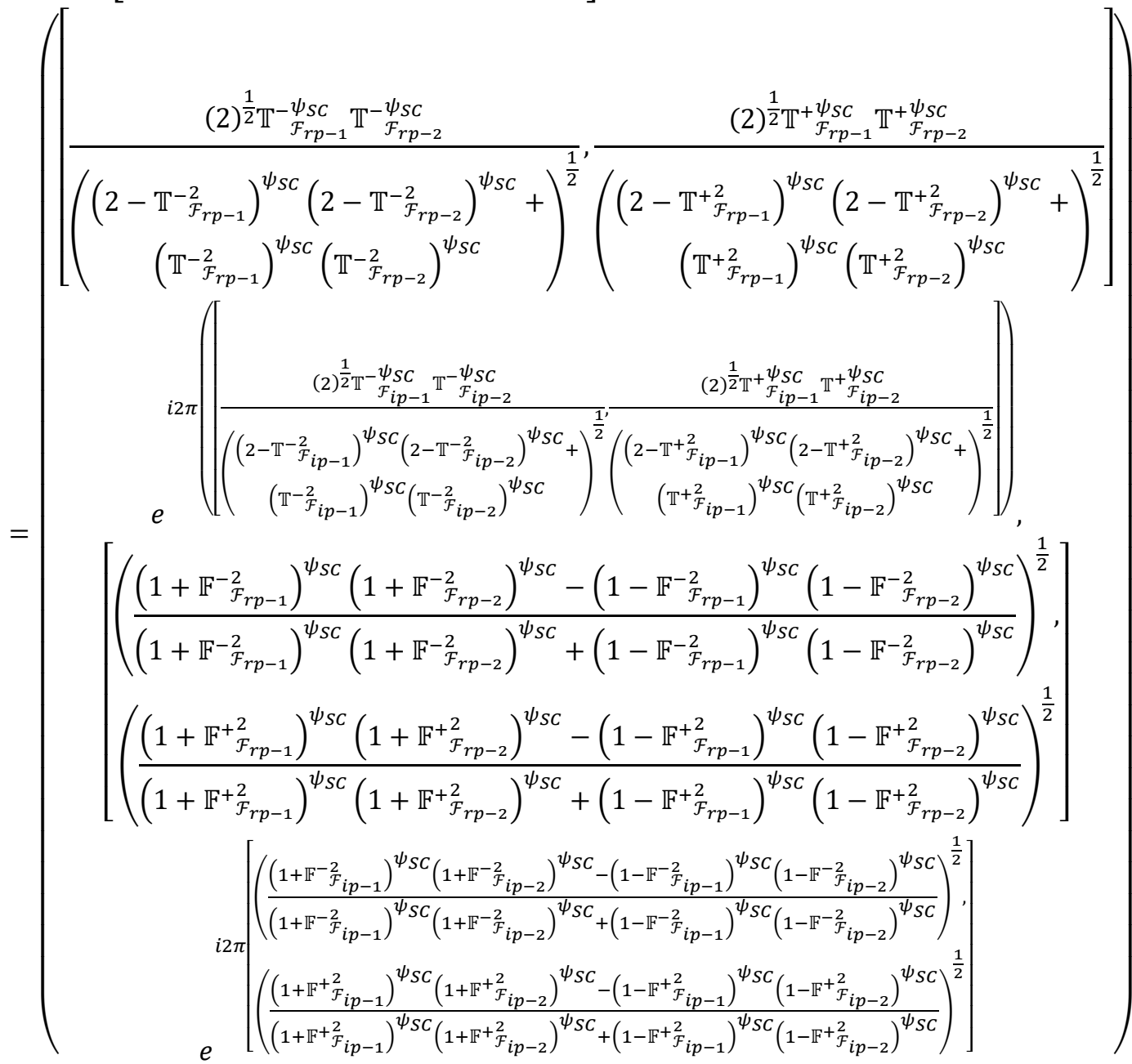

Further, 


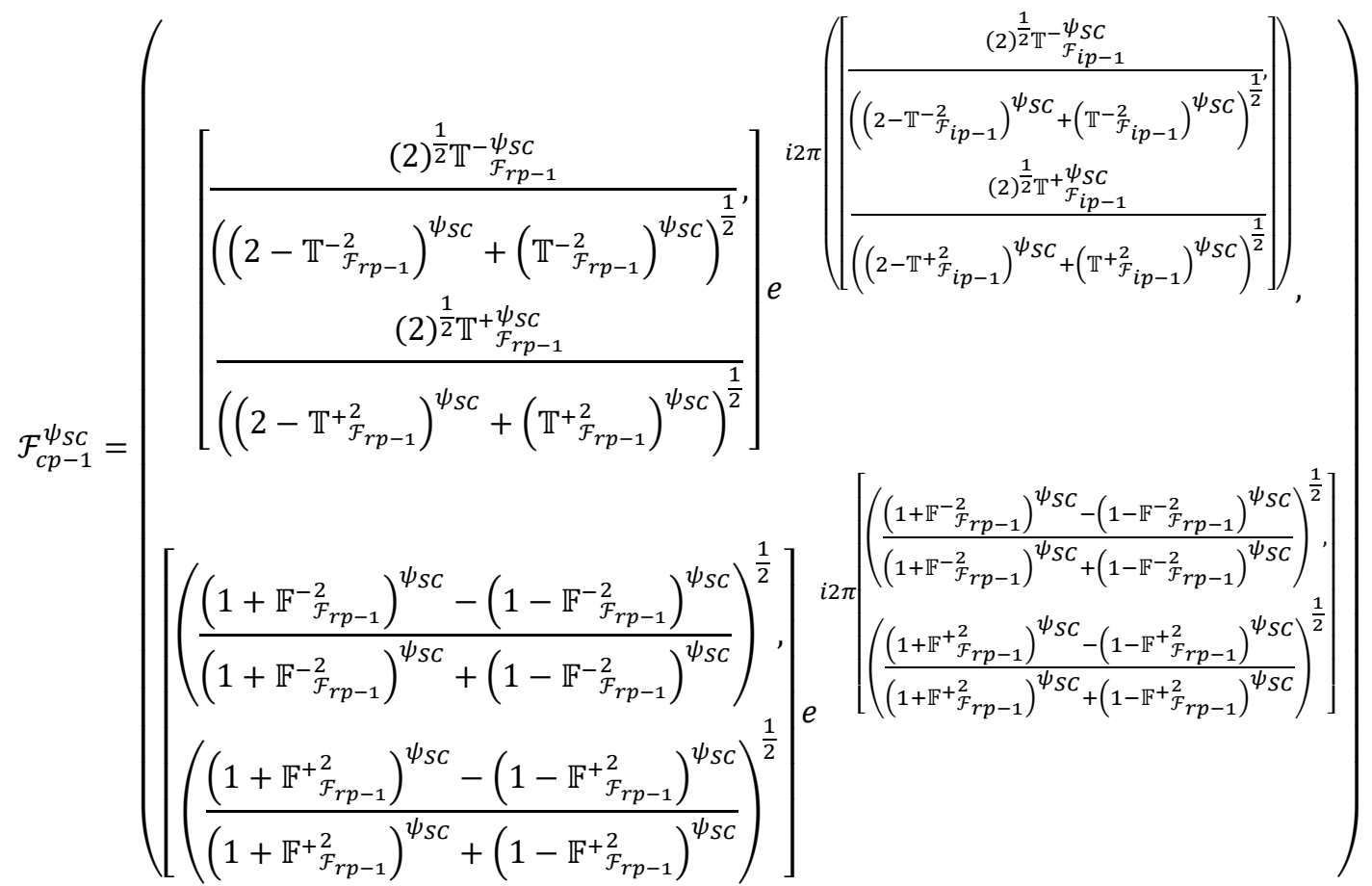

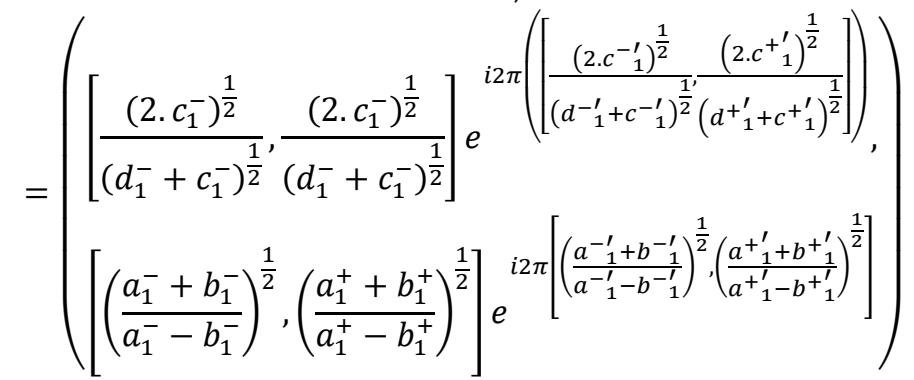

And

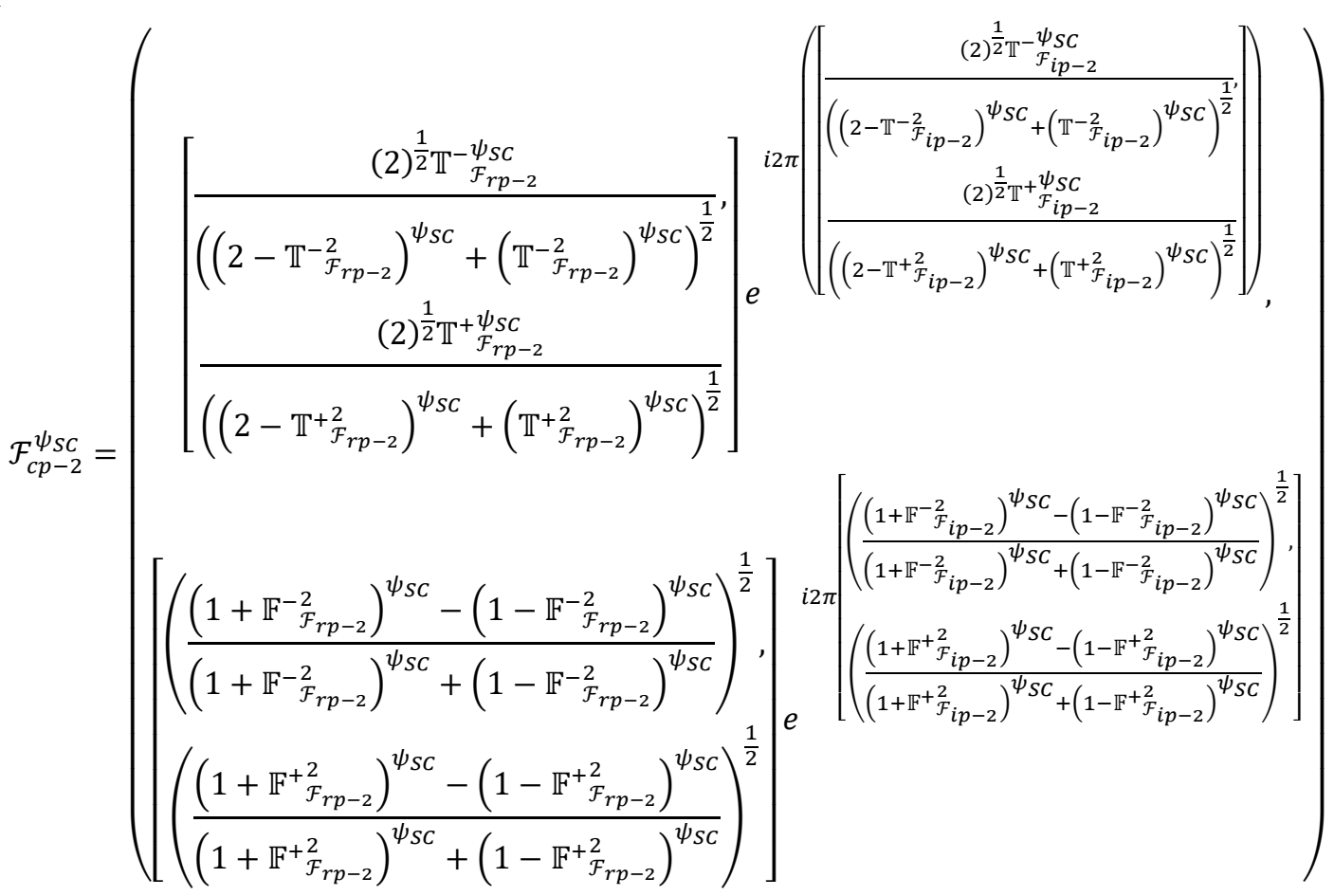




\section{Where}

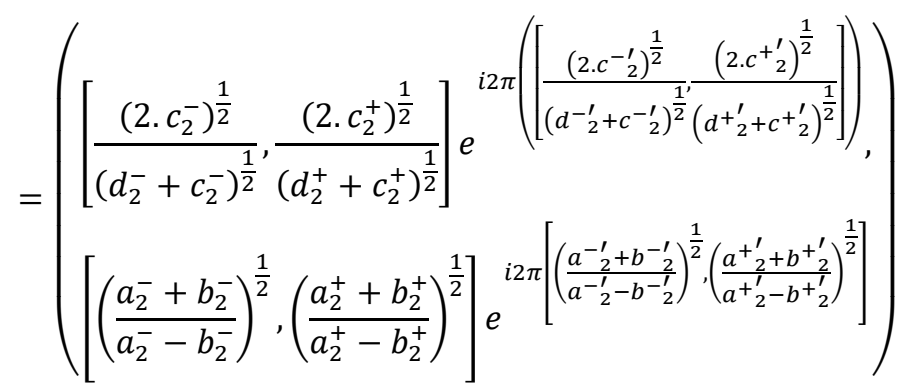

$$
\begin{aligned}
& a_{1}^{-}=\left(1+\mathbb{F}_{\mathcal{F}_{r p-1}}^{-2}\right)^{\psi_{S C}}, b_{1}^{-}=\left(1-\mathbb{F}_{\mathcal{F}_{r p-1}}^{-2}\right)^{\psi_{S C}}, c_{1}^{-}=\left(\mathbb{T}_{\mathcal{F}_{r p-1}}^{-2}\right)^{\psi_{S C}}, d_{1}^{-}=\left(2-\mathbb{T}_{\mathcal{F}_{r p-1}}^{-2}\right)^{\psi_{S C}}, a_{2}^{-}= \\
& \left(1+\mathbb{F}_{\mathcal{F}_{r p-2}}^{-2}\right)^{\psi_{S C}}, b_{2}^{-}=\left(1-\mathbb{F}_{\mathcal{F}_{r p-2}}^{-2}\right)^{\psi_{S C}}, c_{2}^{-}=\left(\mathbb{T}_{\mathcal{F}_{r p-2}}^{-2}\right)^{\psi_{S C}}, d_{2}^{-}=\left(2-\mathbb{T}_{\mathcal{F}_{r p-2}}^{-2}\right)^{\psi_{S C}}, a_{1}^{+}= \\
& \left(1+\mathbb{F}_{\mathcal{F}_{r p-1}}^{2}\right)^{\psi_{S C}}, b_{1}^{+}=\left(1-\mathbb{F}_{\mathcal{F}_{r p-1}}^{+2}\right)^{\psi_{S C}}, c_{1}^{+}=\left(\mathbb{T}_{\mathcal{F}_{r p-1}}^{2}\right)^{\psi_{S C}}, d_{1}^{+}=\left(2-\mathbb{T}_{\mathcal{F}_{r p-1}}^{2}\right)^{\psi_{S C}}, a_{2}^{+}= \\
& \left(1+\mathbb{F}_{\mathcal{F}_{r p-2}}^{2}\right)^{\psi_{S C}}, b_{2}^{+}=\left(1-\mathbb{F}_{\mathcal{F}_{r p-2}}^{2}\right)^{\psi_{S C}}, c_{2}^{+}=\left(\mathbb{T}_{\mathcal{F}_{r p-2}}^{2}\right)^{\psi_{S C}}, d_{2}^{+}=\left(2-\mathbb{T}_{\mathcal{F}_{r p-2}}^{2}\right)^{\psi_{S C}} \quad \text { and } \quad a_{1}^{-\prime}= \\
& \left(1+\mathbb{F}_{\mathcal{F}_{i p-1}}^{-2}\right)^{\psi_{S C}}, b_{1}^{-\prime}=\left(1-\mathbb{F}_{\mathcal{F}_{i p-1}}^{-2}\right)^{\psi_{S C}}, c_{1}^{-\prime}=\left(\mathbb{T}_{\mathcal{F}_{i p-1}}^{-2}\right)^{\psi_{S C}}, d_{1}^{-\prime}=\left(2-\mathbb{T}_{\mathcal{F}_{i p-1}}^{-2}\right)^{\psi_{S C}}, a_{2}^{-\prime}= \\
& \left(1+\mathbb{F}_{\mathcal{F}_{i p-2}}^{-2}\right)^{\psi_{S C}}, b_{2}^{-^{\prime}}=\left(1-\mathbb{F}_{\mathcal{F}_{i p-2}}^{-2}\right)^{\psi_{S C}}, c_{2}^{-_{2}^{\prime}}=\left(\mathbb{T}_{\mathcal{F}_{i p-2}}^{-2}\right)^{\psi_{S C}}, d_{2}^{-^{\prime}}=\left(2-\mathbb{T}_{\mathcal{F}_{i p-2}}^{-2}\right)^{\psi_{S C}}, a^{+^{\prime}}{ }_{1}= \\
& \left(1+\mathbb{F}_{\mathcal{F}_{i p-1}}^{+2}\right)^{\psi_{S C}}, b^{+^{\prime}}{ }_{1}=\left(1-\mathbb{F}_{\mathcal{F}_{i p-1}}^{+2}\right)^{\psi_{S C}}, c^{+^{\prime}}=\left(\mathbb{T}_{\mathcal{F}_{i p-1}}^{+2}\right)^{\psi_{S C}}, d^{+^{\prime}}{ }_{1}=\left(2-\mathbb{T}_{\mathcal{F}_{i p-1}}^{2}\right)^{\psi_{S C}}, a^{+^{\prime}}{ }_{2}= \\
& \left(1+\mathbb{F}_{\mathcal{F}_{i p-2}}^{2}\right)^{\psi_{S C}}, b^{+^{\prime}}{ }_{2}=\left(1-\mathbb{F}_{\mathcal{F}_{i p-2}}^{2}\right)^{\psi_{S C}}, c^{+^{\prime}}{ }_{2}=\left(\mathbb{T}_{\mathcal{F}_{i p-2}}^{2}\right)^{\psi_{S C}}, d^{+^{\prime}}{ }_{2}=\left(2-\mathbb{T}_{\mathcal{F}_{i p-2}}^{2}\right)^{\psi_{S C}} \text {, then }
\end{aligned}
$$

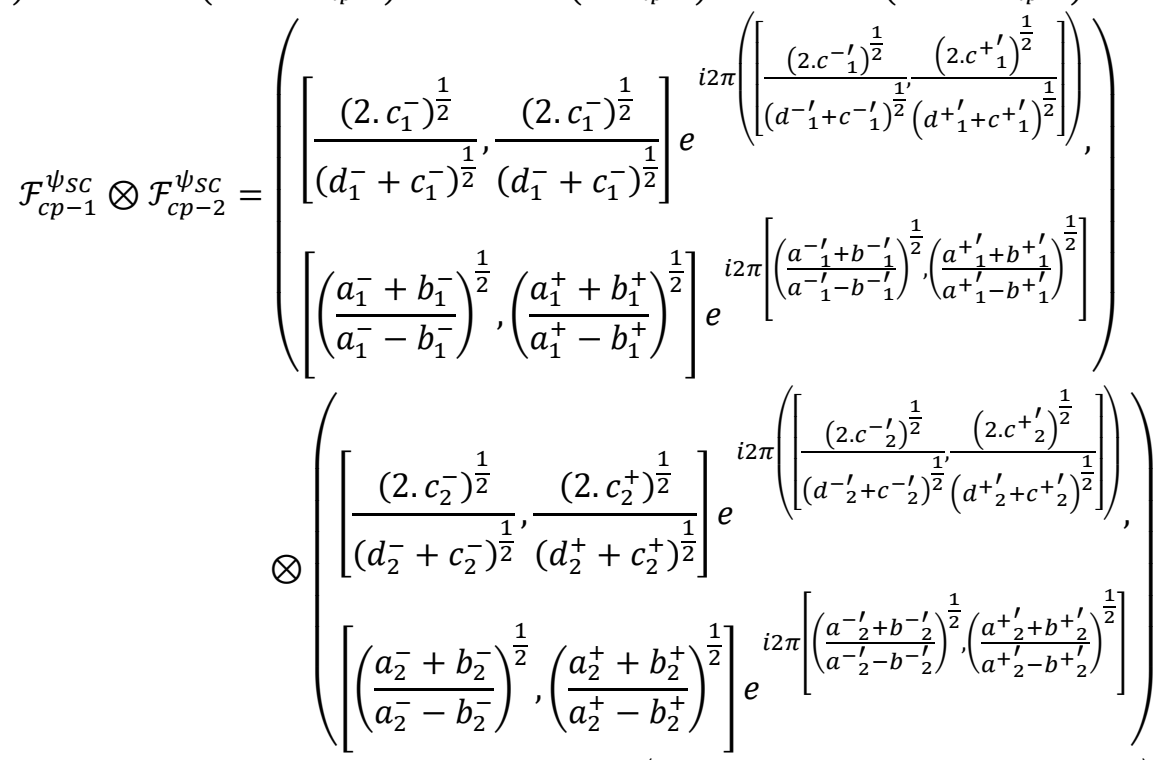

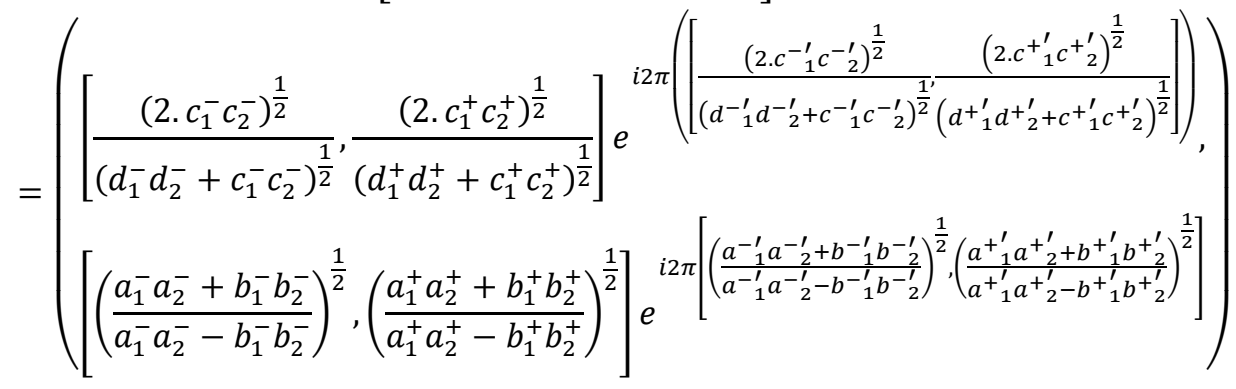




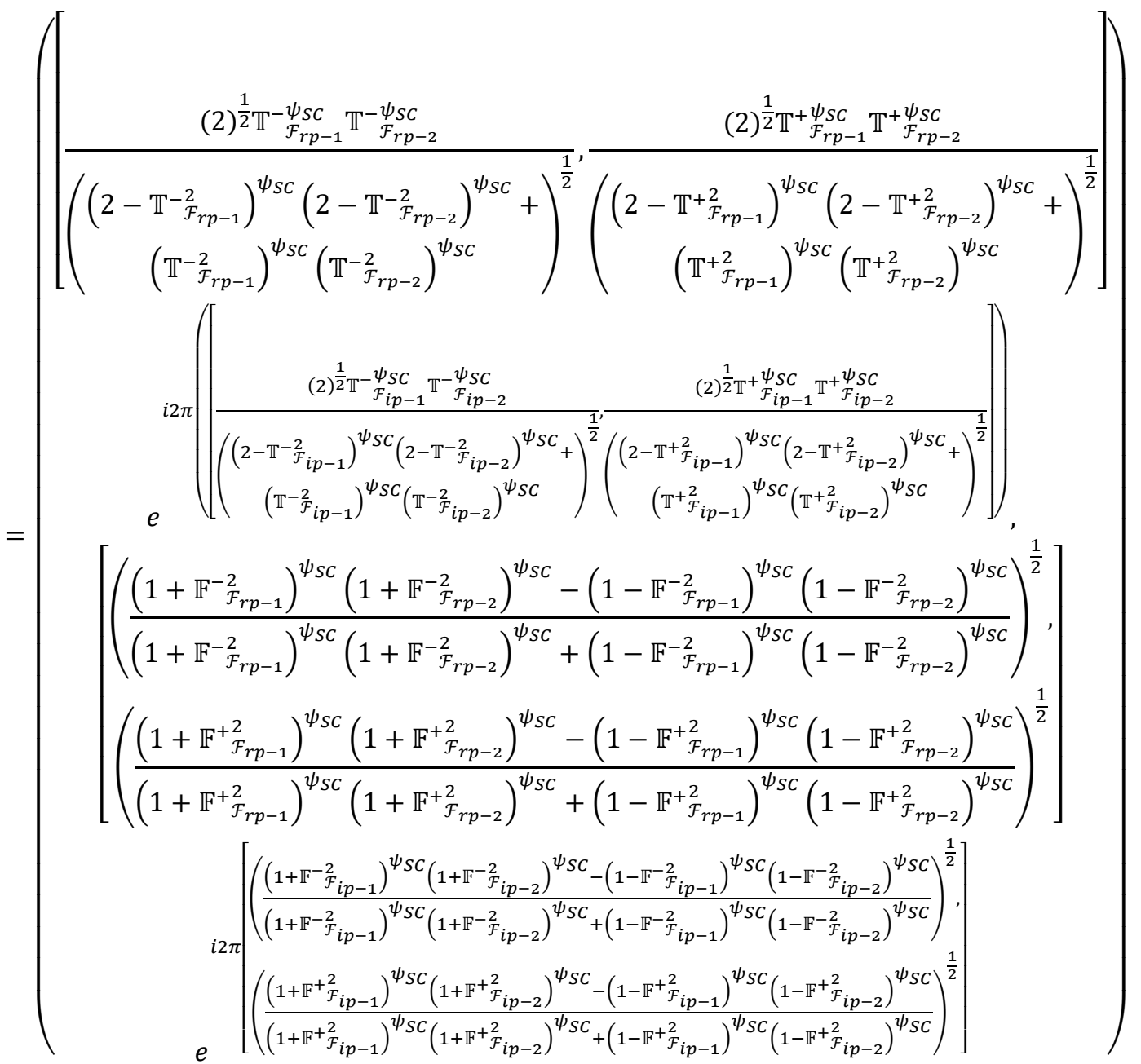

Hence, $\left(\mathcal{F}_{c p-1} \otimes \mathcal{F}_{c p-2}\right)^{\psi_{S C}}=\mathcal{F}_{c p-1}^{\psi_{S C}} \otimes \mathcal{F}_{c p-2}^{\psi_{S C}}$.

4. Trivial.

5. By using the Eq. (19), such that 


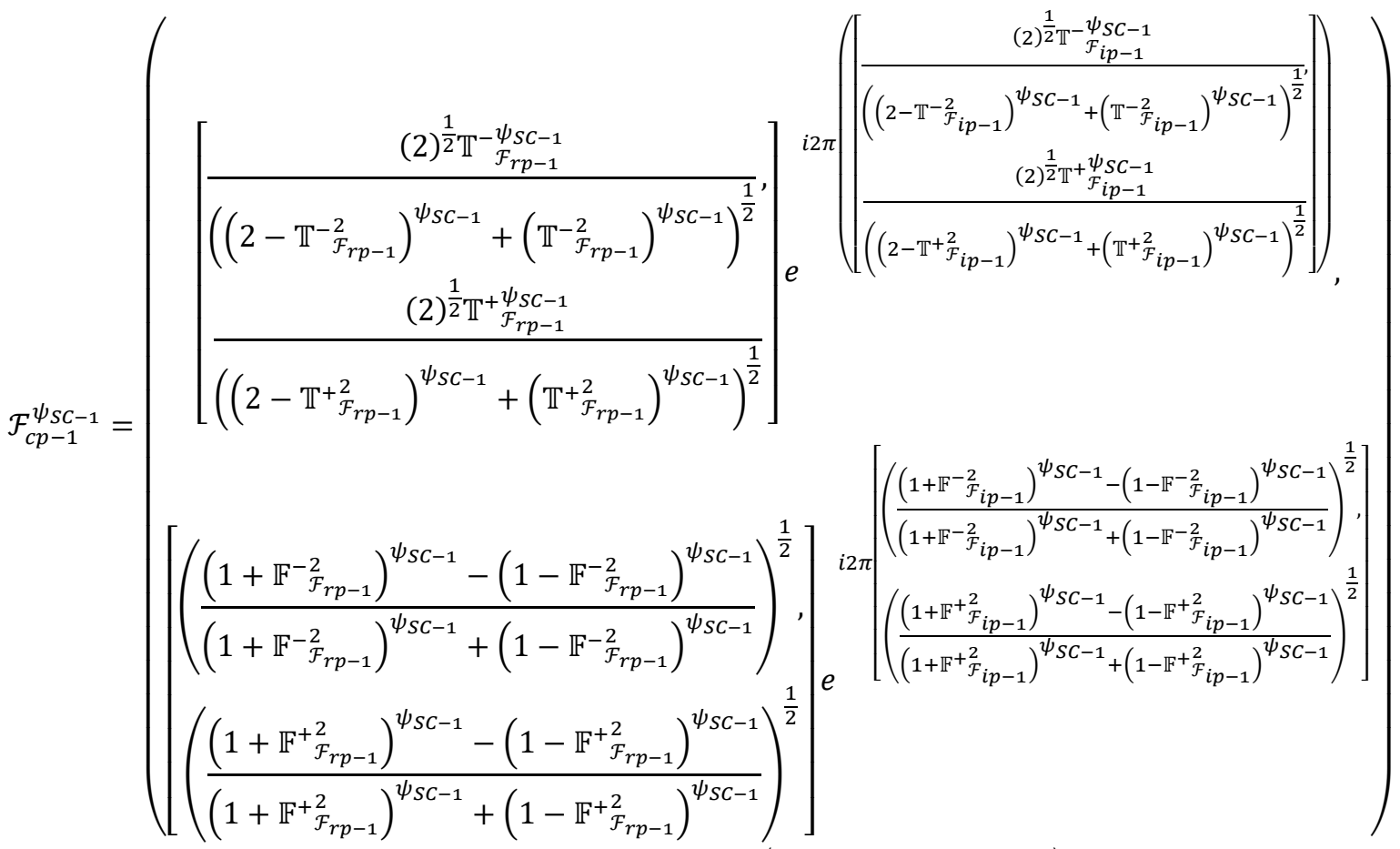

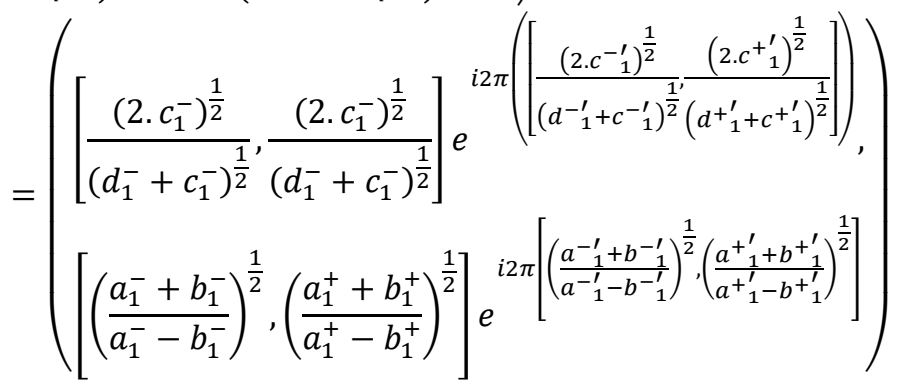

And

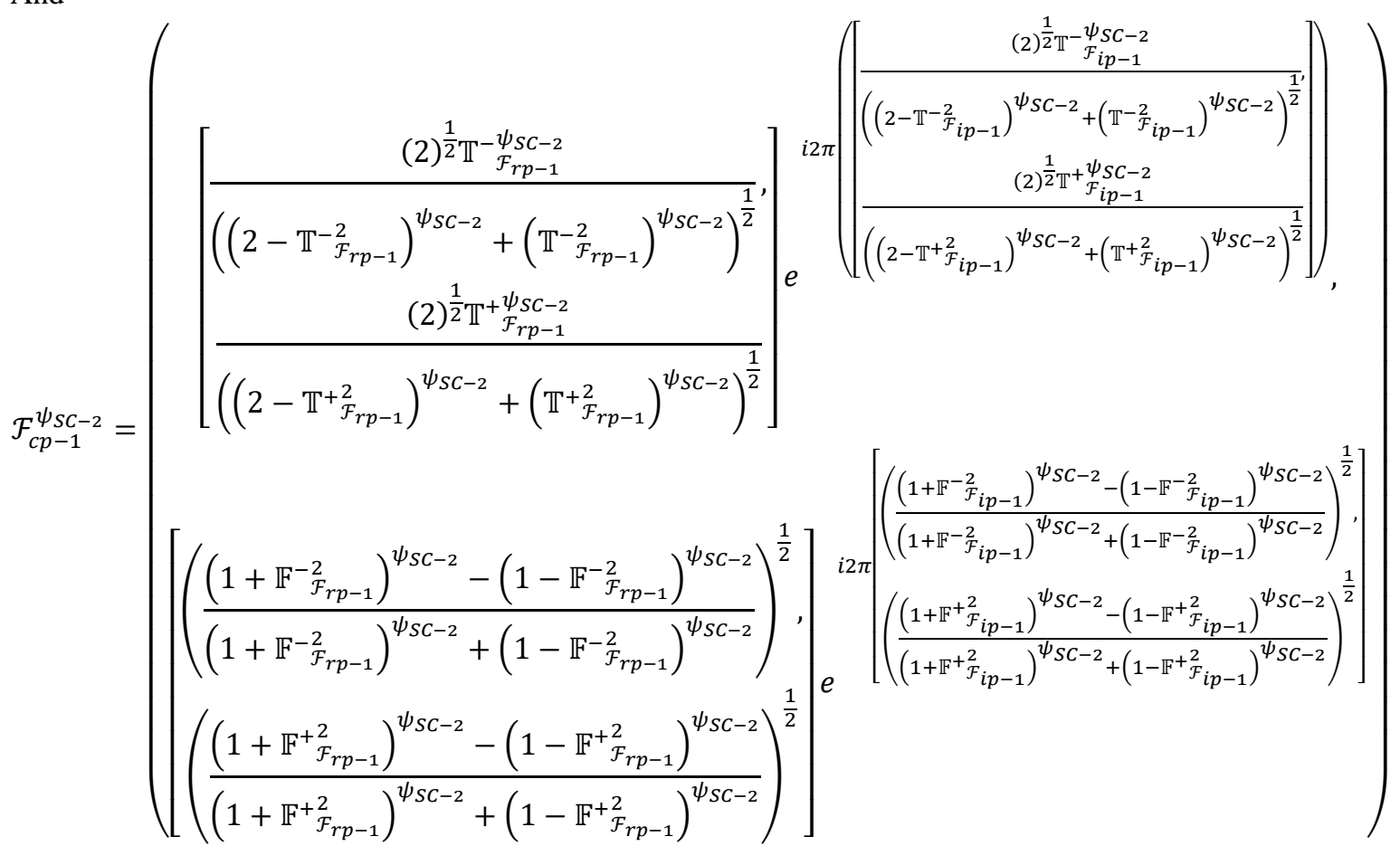




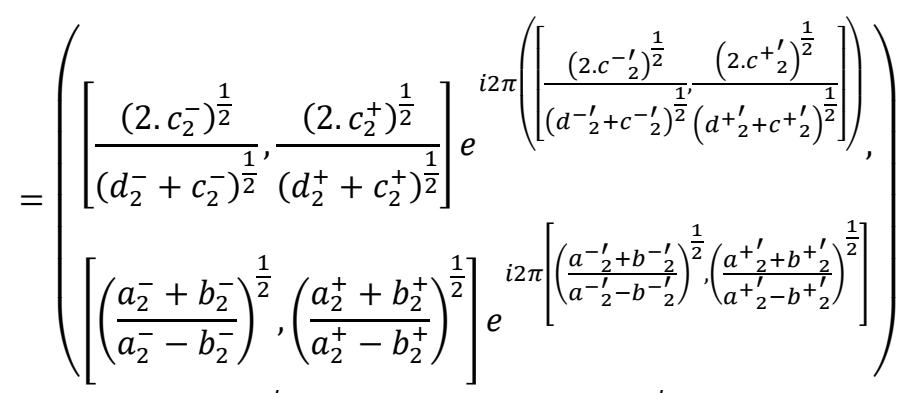

Where $\quad a_{j}^{-}=\left(1+\mathbb{F}_{\mathcal{F}_{r p-1}}^{-2}\right)^{\psi_{S C-j}}, b_{j}^{-}=\left(1-\mathbb{F}_{\mathcal{F}_{r p-1}}^{-2}\right)^{\psi_{S C-j}}, c_{j}^{-}=\left(\mathbb{T}_{\mathcal{F}_{r p-1}}^{-2}\right)^{\psi_{S C-j}}, d_{j}^{-}=(2-$ $\left.\mathbb{T}^{-2} \mathcal{F}_{r p-1}\right)^{\psi_{S C-j}}, j=1,2$ and ${a^{-}}_{j}^{\prime}=\left(1+\mathbb{F}_{\mathcal{F}_{i p-1}}^{-2}\right)^{\psi_{S C-j}}, b_{j}^{-\prime}=\left(1-\mathbb{F}_{\mathcal{F}_{i p-1}}^{-2}\right)^{\psi_{S C-j}}, c^{-\prime}{ }_{j}^{\prime}=\left(\mathbb{T}_{\mathcal{F}_{i p-1}}^{-2}\right)^{\psi_{S C-j}}, d^{-\prime}{ }_{j}^{\prime}=$ $\left(2-\mathbb{T}^{-2} \mathcal{F}_{i p-1}\right)^{\psi_{S C-j}}, a_{j}^{+}=\left(1+\mathbb{F}_{\mathcal{F}_{r p-1}}^{2}\right)^{\psi_{S C-j}}, b_{j}^{+}=\left(1-\mathbb{F}^{+2}{ }_{\mathcal{F}_{r p-1}}\right)^{\psi_{S C-j}}, c_{j}^{+}=\left(\mathbb{T}^{+2}{ }_{\mathcal{F}_{r p-1}}\right)^{\psi_{S C-j}}, d_{j}^{+}=$ $\left(2-\mathbb{T}_{\mathcal{F}_{r p-1}}^{2}\right)^{\psi_{S C-j}}, j=1,2, \quad$ and $\quad a^{+^{\prime}}=\left(1+\mathbb{F}_{\mathcal{F}_{i p-1}}^{2}\right)^{\psi_{S C-j}}, b^{+_{j}^{\prime}}=\left(1-\mathbb{F}_{\mathcal{F}_{i p-1}}^{2}\right)^{\psi_{S C-j}}, c^{+^{\prime}}{ }_{j}=$ $\left(\mathbb{T}^{+2}{ }_{\mathcal{F}_{i p-1}}\right)^{\psi_{S C-j}}, d^{+^{\prime}}=\left(2-\mathbb{T}^{+2}{ }_{\mathcal{F}_{i p-1}}\right)^{\psi_{S C-j}}, j=1,2$, then $\mathcal{F}_{c p-1}^{\psi_{S C-1}} \oplus \mathcal{F}_{c p-1}^{\psi_{S C-2}}$

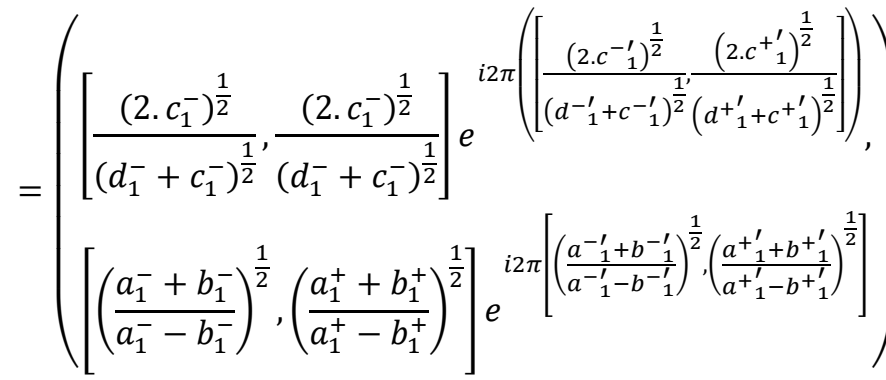

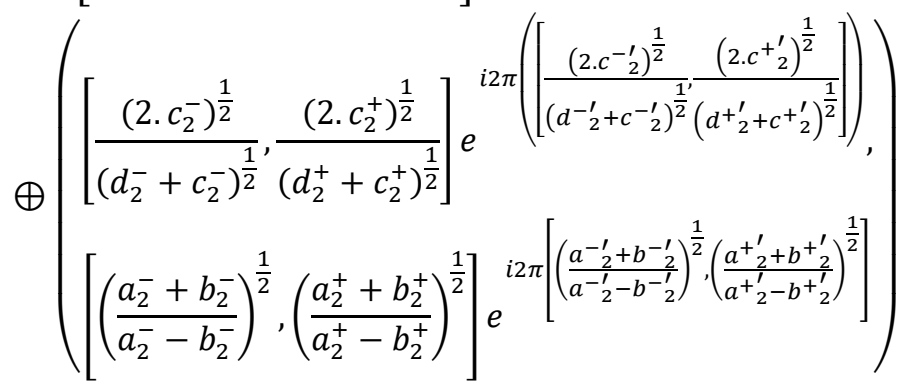

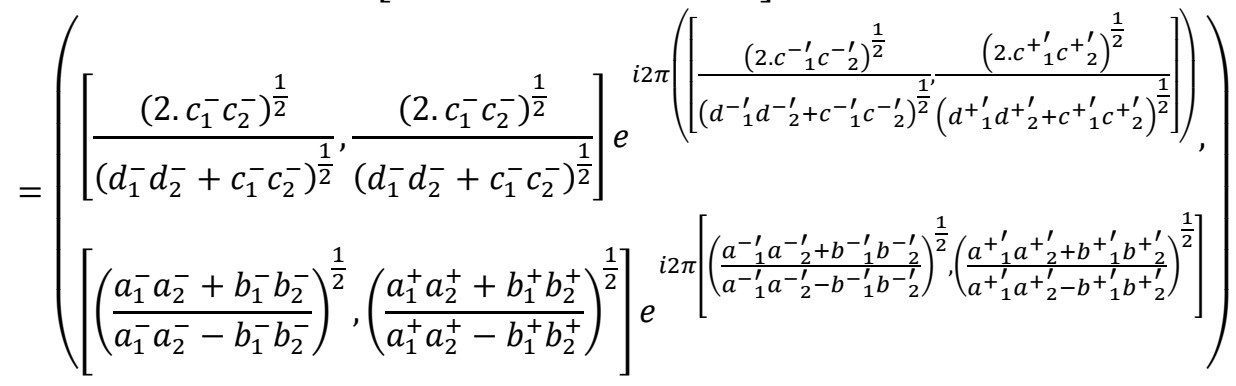




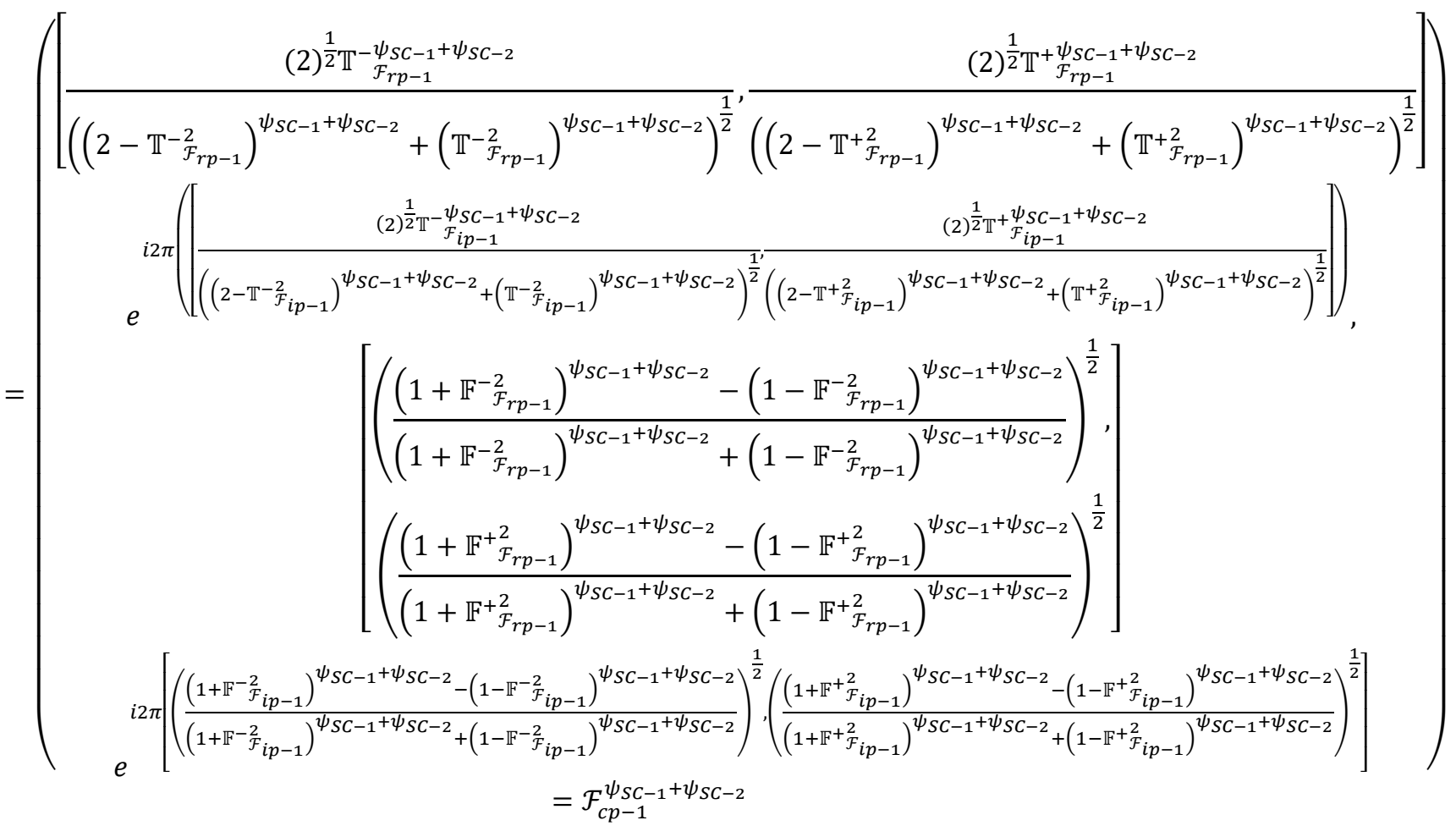

The result is proved.

\section{Complex Interval-Valued Pythagorean Fuzzy Einstein Geometric Aggregation Operators}

In the previous section, we explored some Einstein laws by using the CIVPFSs. The goal of this study, by using the elaborated laws, we explore the CIVPFEWG, CIVPFEOWG operators, and utilized their special cases.

Definition 4: Let $\mathcal{F}_{c p-j}=\left(\left[\mathbb{T}_{\mathcal{F}_{r p-j}}^{-}, \mathbb{T}_{\mathcal{F}_{r p-j}}^{+}\right] e^{i 2 \pi\left(\left[\mathbb{T}_{\mathcal{F}_{i p-j}}^{-}, \mathbb{T}_{\mathcal{F}_{i p-j}}^{+}\right]\right)},\left[\mathbb{F}_{\mathcal{F}_{r p-j}}^{-}, \mathbb{F}_{\mathcal{F}_{r p-j}}^{+}\right] e^{i 2 \pi\left(\left[\mathbb{F}_{\mathcal{F}_{i p-j}}^{-}, \mathbb{F}_{\mathcal{F}_{i p-j}}^{+}\right]\right)}\right), j=$

$1,2, \ldots, \mathbb{m}_{l e}$ be a collection of CIVPFNs weight vector $\Omega=\left\{\Omega_{1}, \Omega_{2}, \ldots, \Omega_{\mathbb{m}_{l e}}\right\}$ with a rule that is $\sum_{j=1}^{\mathbb{m}_{l e}} \Omega_{j}=1$.

Then the CIVPFEWG operator is initiated by:

$\operatorname{CIVPFEWG}\left(\mathcal{F}_{c p-1}, \mathcal{F}_{c p-2}, \ldots, \mathcal{F}_{c p-\mathbb{m}} l e=\bigotimes_{j=1}^{\mathrm{m}_{l e}}\left(\mathcal{F}_{c p-j}^{\Omega_{j}}\right)\right.$

If we fixed the value of $\Omega_{j}=\frac{1}{\mathrm{~m}_{l e}}$ for all $j$, then the CIVPFEWG operator is converted for complex intervalvalued Pythagorean fuzzy geometric (CIVPFG) operator such that

$\operatorname{CIVPFG}\left(\mathcal{F}_{c p-1}, \mathcal{F}_{c p-2}, \ldots, \mathcal{F}_{c p-\mathbb{m}_{l e}}\right)=\left(\bigotimes_{j=1}^{\mathrm{m}_{l} l e}\left(\mathcal{F}_{c p-j}\right)\right)^{\frac{1}{\mathrm{~m}_{l} e}}$

Theorem 2: Let $\mathcal{F}_{c p-j}=\left(\left[\mathbb{T}_{\mathcal{F}_{r p-j}}^{-}, \mathbb{T}_{\mathcal{F}_{r p-j}}^{+}\right] e^{i 2 \pi\left(\left[\mathbb{T}_{\mathcal{F}_{i p-j}}^{-} \mathbb{T}_{\mathcal{F}_{i p-j}}^{+}\right]\right)},\left[\mathbb{F}_{\mathcal{F}_{r p-j}}^{-}, \mathbb{F}_{\mathcal{F}_{r p-j}}^{+}\right] e^{i 2 \pi\left(\left[\mathbb{F}_{\mathcal{F}_{i p-j}}, \mathbb{F}_{\mathcal{F}_{i p-j}}^{+}\right]\right)}\right), j=$

$1,2, \ldots, \mathbb{m}_{l e}$ be a collection of CIVPFNs weight vector $\Omega=\left\{\Omega_{1}, \Omega_{2}, \ldots, \Omega_{\mathbb{m}_{l e}}\right\}$ with a rule that is $\sum_{j=1}^{\mathbb{m}_{l e}} \Omega_{j}=1$.

Then by using Eq. (20), we determine 


$$
\begin{aligned}
& \operatorname{CIVPFEWG}\left(\mathcal{F}_{c p-1}, \mathcal{F}_{c p-2}, \ldots, \mathcal{F}_{c p-\mathrm{m}_{l e}}\right)=
\end{aligned}
$$

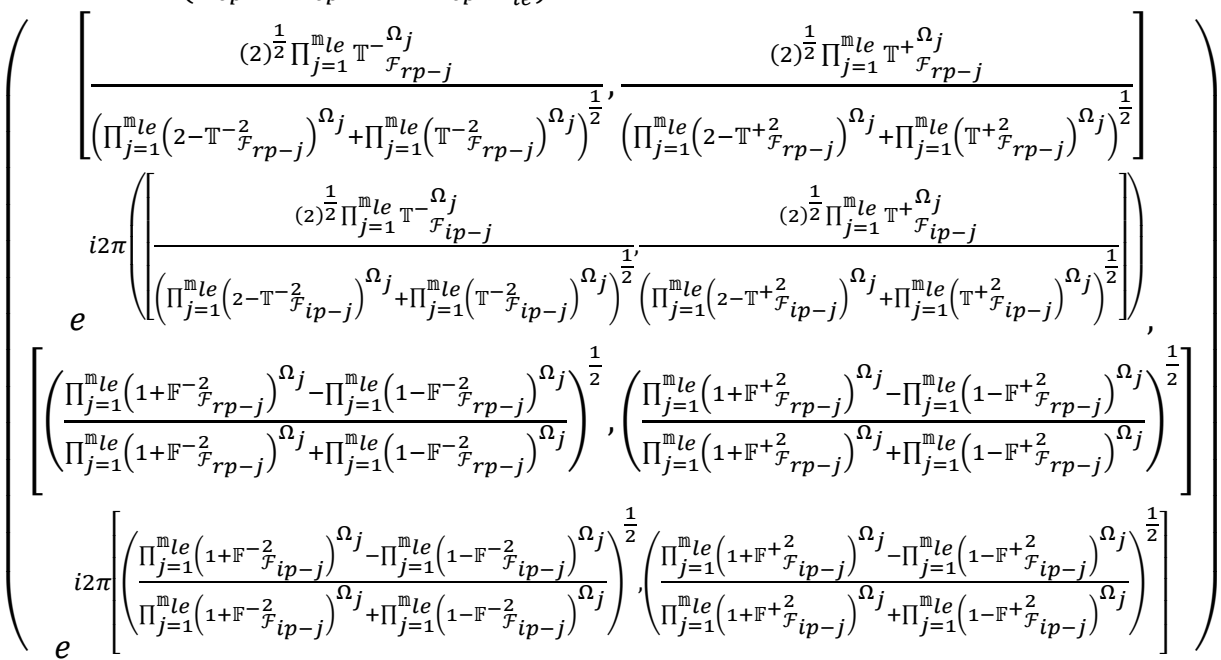

Proof: By using mathematical induction, we prove Eq. (22), for this we choose $\mathbb{m}_{l e}=2$, we determine By using Eq. (19), we know that

$$
\operatorname{CIVPFEWG}\left(\mathcal{F}_{c p-1}, \mathcal{F}_{c p-2}\right)=\mathcal{F}_{c p-1}^{\Omega_{1}} \otimes \mathcal{F}_{c p-2}^{\Omega_{2}}
$$

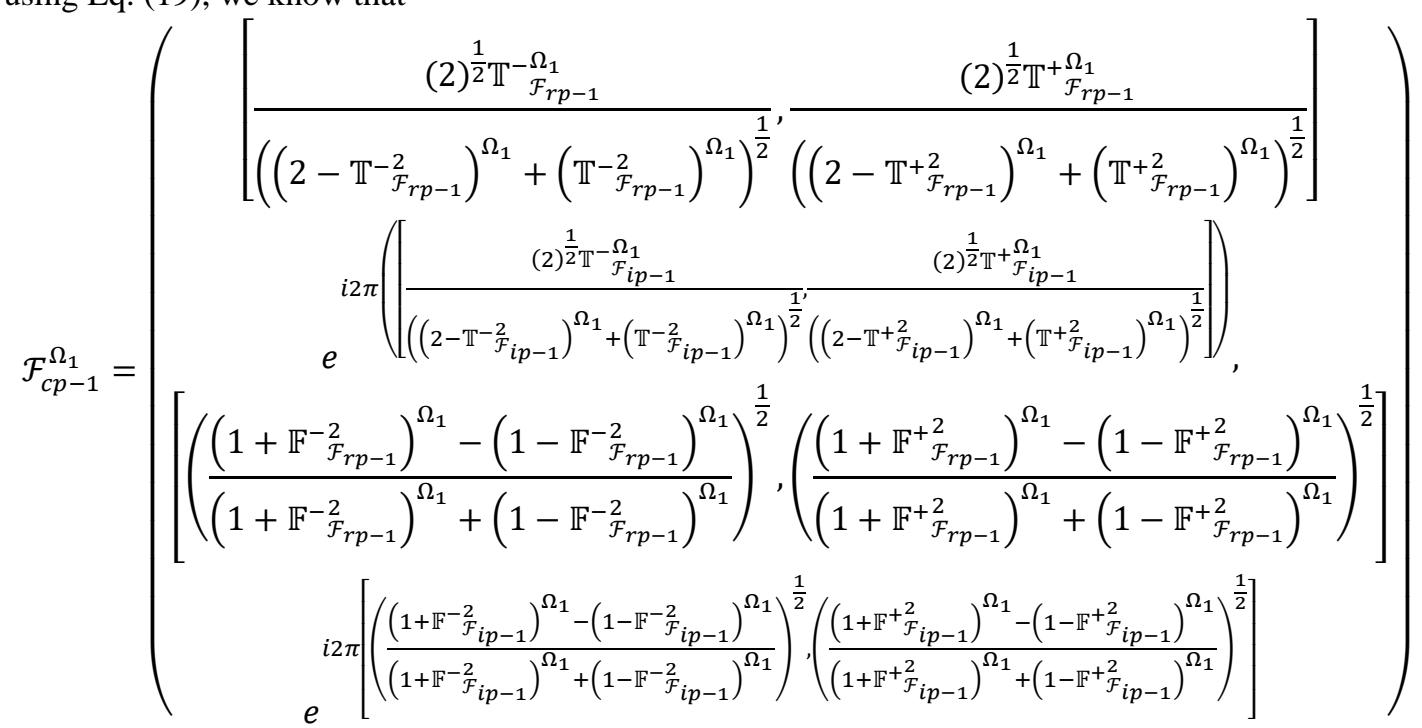

And

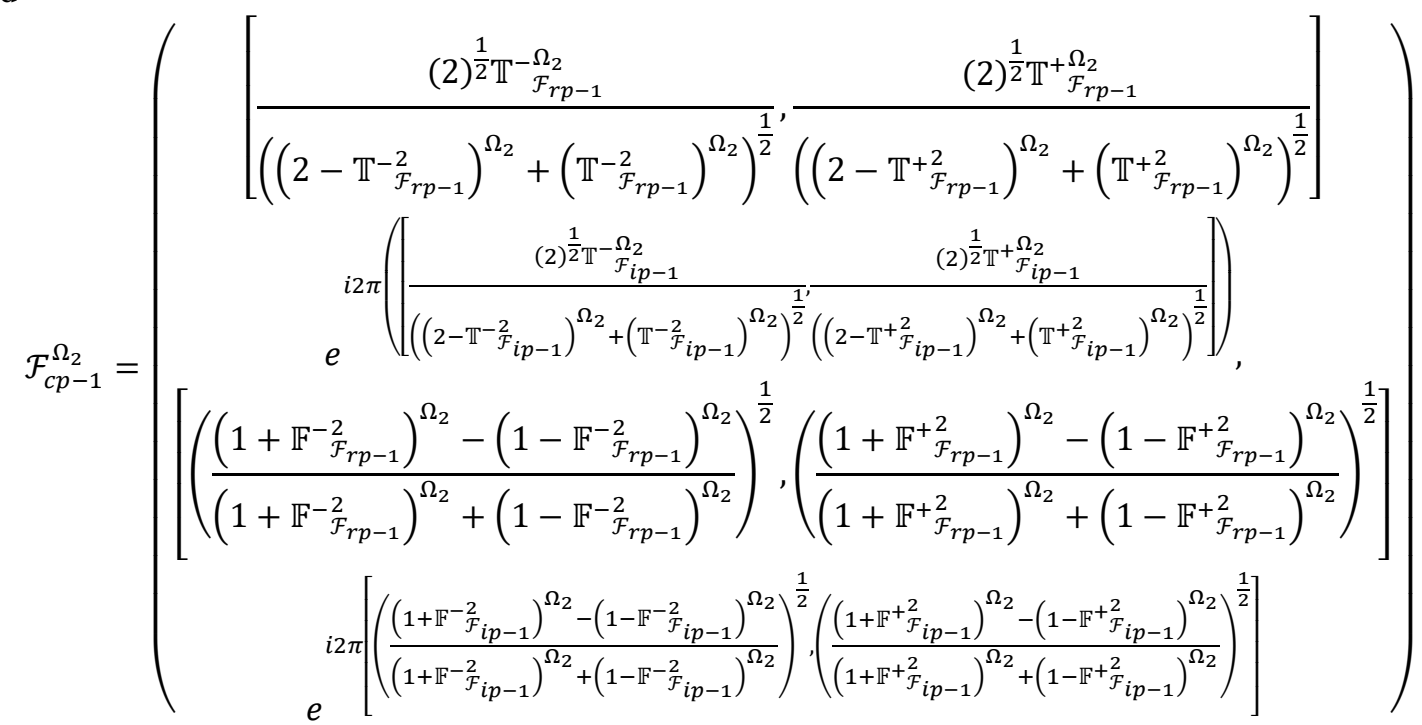

Then 


$$
\begin{aligned}
& \operatorname{CIVPFEWG}\left(\mathcal{F}_{c p-1}, \mathcal{F}_{c p-2}\right)=\mathcal{F}_{c p-1}^{\Omega_{1}} \otimes \mathcal{F}_{c p-2}^{\Omega_{2}} \\
& \left(\left[\frac{(2)^{\frac{1}{2}} \mathbb{T}^{-\Omega_{\mathcal{F}_{r p-1}}}}{\left(\left(2-\mathbb{T}^{-2} \mathcal{F}_{r p-1}\right)^{\Omega_{1}}+\left(\mathbb{T}^{-2}{ }_{\mathcal{F}_{r p-1}}\right)^{\Omega_{1}}\right)^{\frac{1}{2}}}, \frac{(2)^{\frac{1}{2} \mathbb{T}^{+}} \mathcal{F}_{\mathcal{F}_{r p-1}}^{\Omega_{1}}}{\left(\left(2-\mathbb{T}^{+}{ }_{\mathcal{F}_{r p-1}}\right)^{\Omega_{1}}+\left(\mathbb{T}^{+}{ }_{\mathcal{F}_{r p-1}}\right)^{\Omega_{1}}\right)^{\frac{1}{2}}}\right]\right. \\
& e^{i 2 \pi}\left(\left[\frac{(2)^{\frac{1}{2}} \mathbb{T}^{-} \mathcal{F}_{i p-1}}{\left(\left(2-\mathbb{T}^{-\frac{2}{\mathcal{F}_{i p-1}}}\right)^{\Omega_{1}}+\left(\mathbb{T}^{-\frac{2}{\mathcal{F}_{i p-1}}}\right)^{\Omega_{1}}\right)^{\frac{1}{2}}} \frac{(2)^{\frac{1}{2}} \mathbb{T}^{+} \mathcal{F}_{i p-1}}{\left.\left(2-\mathbb{T}^{+}{ }_{\mathcal{F}_{i p-1}}^{2}\right)^{\Omega_{1}}+\left(\mathbb{T}^{+}{ }_{\mathcal{F}}^{2}{ }_{i p-1}\right)^{\Omega_{1}}\right)^{\frac{1}{2}}}\right]\right), \\
& =\left[\left[\left(\frac{\left(1+\mathbb{F}_{\mathcal{F}_{r p-1}}^{-2}\right)^{\Omega_{1}}-\left(1-\mathbb{F}_{\mathcal{F}_{r p-1}}^{-2}\right)^{\Omega_{1}}}{\left(1+\mathbb{F}_{\mathcal{F}_{r p-1}}^{-2}\right)^{\Omega_{1}}+\left(1-\mathbb{F}_{\mathcal{F}_{r p-1}}^{-2}\right)^{\Omega_{1}}}\right)^{\frac{1}{2}},\left(\frac{\left(1+\mathbb{F}_{\mathcal{F}_{r p-1}}^{+2}\right)^{\Omega_{1}}-\left(1-\mathbb{F}_{\mathcal{F}_{r p-1}}^{+2}\right)^{\Omega_{1}}}{\left(1+\mathbb{F}_{\mathcal{F}_{r p-1}}^{+2}\right)^{\Omega_{1}}+\left(1-\mathbb{F}_{\mathcal{F}_{r p-1}}^{+2}\right)^{\Omega_{1}}}\right)^{\frac{1}{2}}\right]\right.
\end{aligned}
$$

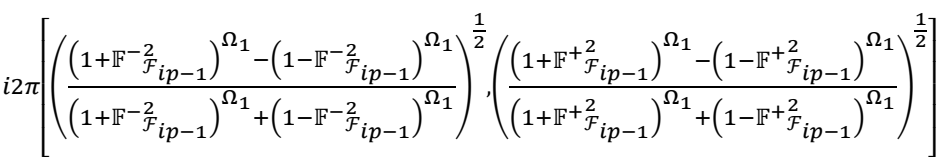

$$
\begin{aligned}
& {\left[\frac{(2)^{\frac{1}{2}} \mathbb{T}_{\mathcal{F}_{r p-1}}^{-\Omega_{2}}}{\left(\left(2-\mathbb{T}_{\mathcal{F}_{r p-1}}^{-2}\right)^{\Omega_{2}}+\left(\mathbb{T}^{-2}{ }_{\mathcal{F}_{r p-1}}\right)^{\Omega_{2}}\right)^{\frac{1}{2}}}, \frac{(2)^{\frac{1}{2}} \mathbb{T}^{+\mathcal{F}_{r p-1}}}{\left(\left(2-\mathbb{T}^{+}{ }_{\mathcal{F}_{r p-1}}^{2}\right)^{\Omega_{2}}+\left(\mathbb{T}^{+}{ }_{\mathcal{F}_{r p-1}}^{2}\right)^{\Omega_{2}}\right)^{\frac{1}{2}}}\right]} \\
& e^{i 2 \pi}\left(\left[\frac{(2)^{\frac{1}{2}} \mathbb{T}_{\mathcal{F}_{i p-1}}^{-\Omega_{2}}}{\left(\left(2-\mathbb{T}^{-\frac{2}{\mathcal{F}_{i p-1}}}\right)^{\Omega_{2}}+\left(\mathbb{T}^{-\frac{2}{\mathcal{F}_{i p-1}}}\right)^{\Omega_{2}}\right)^{\frac{1}{2}}\left(\left(2-\mathbb{T}^{+}{ }_{\mathcal{F}_{i p-1}}^{2}\right)^{\Omega_{2}}+\left(\mathbb{T}^{+}{ }_{\mathcal{F}_{i p-1}}^{2}\right)^{\Omega_{2}}\right)^{\frac{1}{2}}}\right]\right), \\
& {\left[\left(\frac{\left(1+\mathbb{F}_{\mathcal{F}_{r p-1}}^{-2}\right)^{\Omega_{2}}-\left(1-\mathbb{F}_{\mathcal{F}_{r p-1}}^{-2}\right)^{\Omega_{2}}}{\left(1+\mathbb{F}_{\mathcal{F}_{r p-1}}^{-2}\right)^{\Omega_{2}}+\left(1-\mathbb{F}_{\mathcal{F}_{r p-1}}^{-2}\right)^{\Omega_{2}}}\right)^{\frac{1}{2}},\left(\frac{\left(1+\mathbb{F}_{\mathcal{F}_{r p-1}}^{+2}\right)^{\Omega_{2}}-\left(1-\mathbb{F}_{\mathcal{F}_{r p-1}}^{+2}\right)^{\Omega_{2}}}{\left(1+\mathbb{F}_{\mathcal{F}_{r p-1}}^{+2}\right)^{\Omega_{2}}+\left(1-\mathbb{F}_{\mathcal{F}_{r p-1}}^{+2}\right)^{\Omega_{2}}}\right)^{\frac{1}{2}}\right]}
\end{aligned}
$$

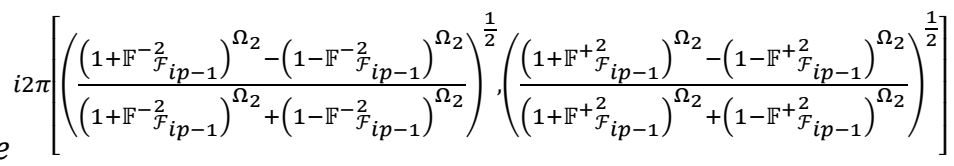

$$
\begin{aligned}
& \left(\left[\frac{(2)^{\frac{1}{2}} \prod_{j=1}^{2} \mathbb{T}_{\mathcal{F}_{r p-j}}^{\Omega_{j}}}{\left(\prod_{j=1}^{2}\left(2-\mathbb{T}^{-2}{ }_{\mathcal{F}_{r p-j}}^{2}\right)^{\Omega_{j}}+\prod_{j=1}^{2}\left(\mathbb{T}^{-2} \mathcal{F}_{r p-j}\right)^{\Omega_{j}}\right)^{\frac{1}{2}}}, \frac{(2)^{\frac{1}{2}} \prod_{j=1}^{2} \mathbb{T}^{+}{ }_{\mathcal{F}_{r p-j}}^{\Omega_{j}}}{\left(\prod_{j=1}^{2}\left(2-\mathbb{T}^{+\mathcal{F}_{r p-j}}\right)^{\Omega_{j}}+\prod_{j=1}^{2}\left(\mathbb{T}^{+}{ }_{\mathcal{F}_{r p-j}}^{2}\right)^{\Omega_{j}}\right)^{\frac{1}{2}}}\right]\right.
\end{aligned}
$$

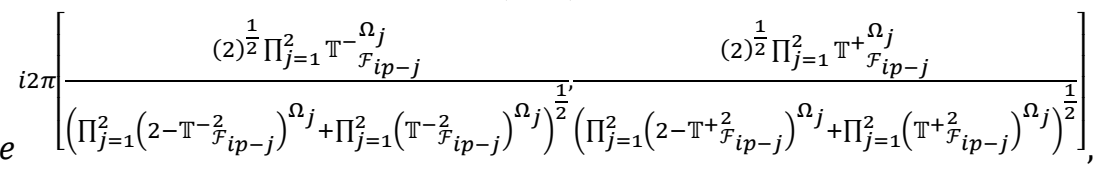

$$
\begin{aligned}
& =\left[\left[\left(\frac{\prod_{j=1}^{2}\left(1+\mathbb{F}_{\mathcal{F}_{r p-j}}^{-2}\right)^{\Omega_{j}}-\prod_{j=1}^{2}\left(1-\mathbb{F}_{\mathcal{F}_{r p-j}}^{-2}\right)^{\Omega_{j}}}{\prod_{j=1}^{2}\left(1+\mathbb{F}_{\mathcal{F}_{r p-j}}^{-2}\right)^{\Omega_{j}}+\prod_{j=1}^{2}\left(1-\mathbb{F}_{\mathcal{F}_{r p-j}}^{-2}\right)^{\Omega_{j}}}\right)^{\frac{1}{2}},\left(\frac{\prod_{j=1}^{2}\left(1+{F^{+}}_{\mathcal{F}_{r p-j}}^{2}\right)^{\Omega_{j}}-\prod_{j=1}^{2}\left(1-{F^{+}}_{\mathcal{F}_{r p-j}}^{2}\right)^{\Omega_{j}}}{\prod_{j=1}^{2}\left(1+\mathbb{F}_{\mathcal{F}_{r p-j}}^{+2}\right)^{\Omega_{j}}+\prod_{j=1}^{2}\left(1-{F^{+}}_{\mathcal{F}_{r p-j}}^{2}\right)^{\Omega_{j}}}\right]\right.\right.
\end{aligned}
$$

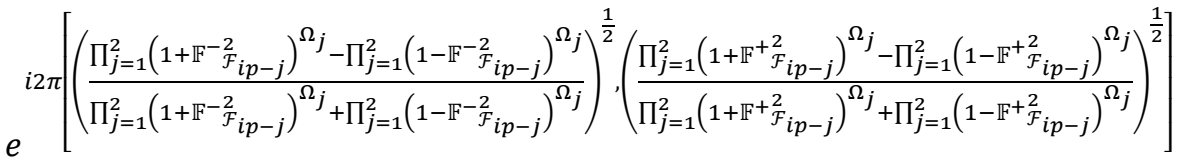

We assume that Eq. (22), is true for $\mathbb{m}_{l e}=k$, such that

$$
\operatorname{CIVPFEWG}\left(\mathcal{F}_{c p-1}, \mathcal{F}_{c p-2}, \ldots, \mathcal{F}_{c p-k}\right)
$$




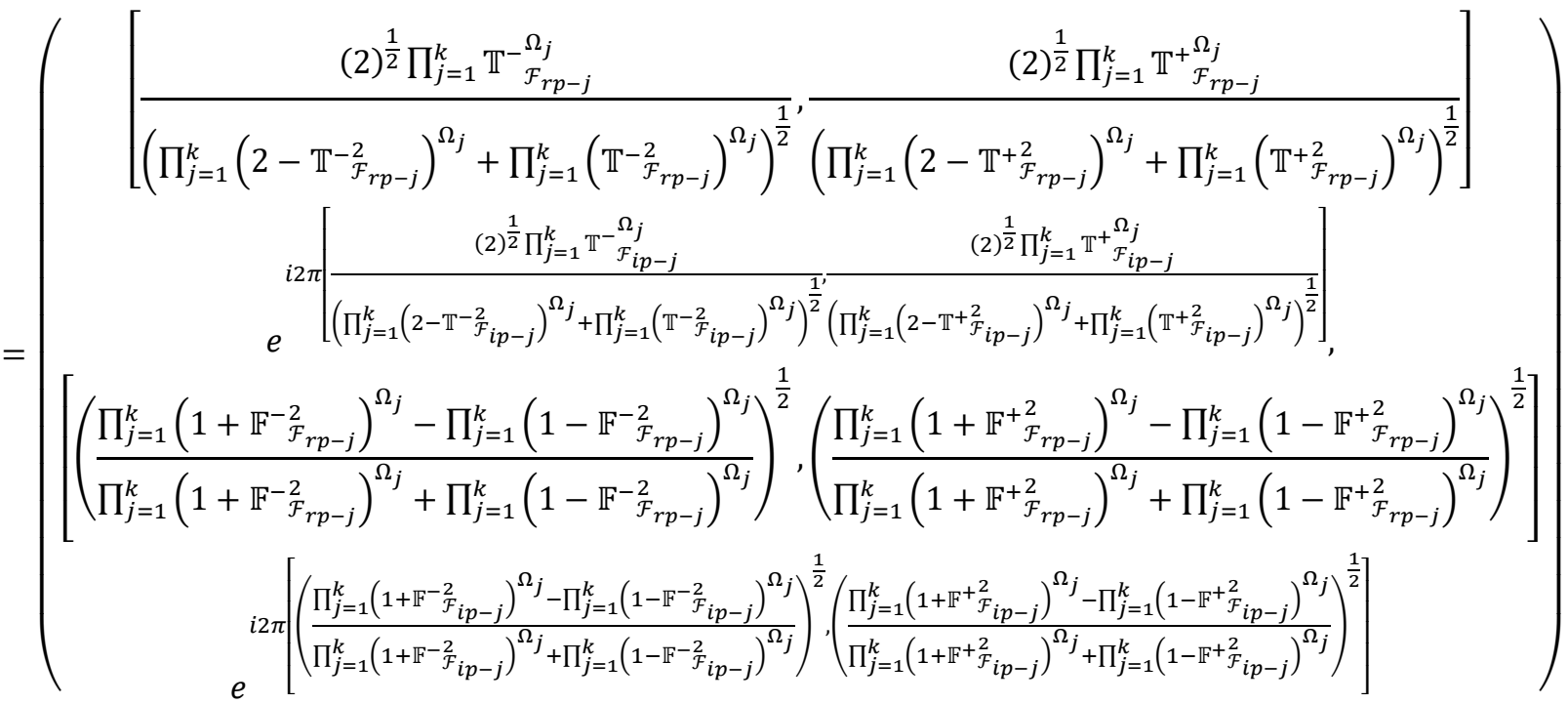

Then, we will prove that Eq. (22) also holds for $\mathrm{m}_{l e}=k+1$, such that

$$
\begin{aligned}
& \operatorname{CIVPFEWG}\left(\mathcal{F}_{c p-1}, \mathcal{F}_{c p-2}, \ldots, \mathcal{F}_{c p-k+1}\right)=\operatorname{CIVPFEWG}\left(\mathcal{F}_{c p-1}, \mathcal{F}_{c p-2}, \ldots, \mathcal{F}_{c p-k}\right) \otimes \mathcal{F}_{c p-k+1}^{\Omega_{k+1}}
\end{aligned}
$$

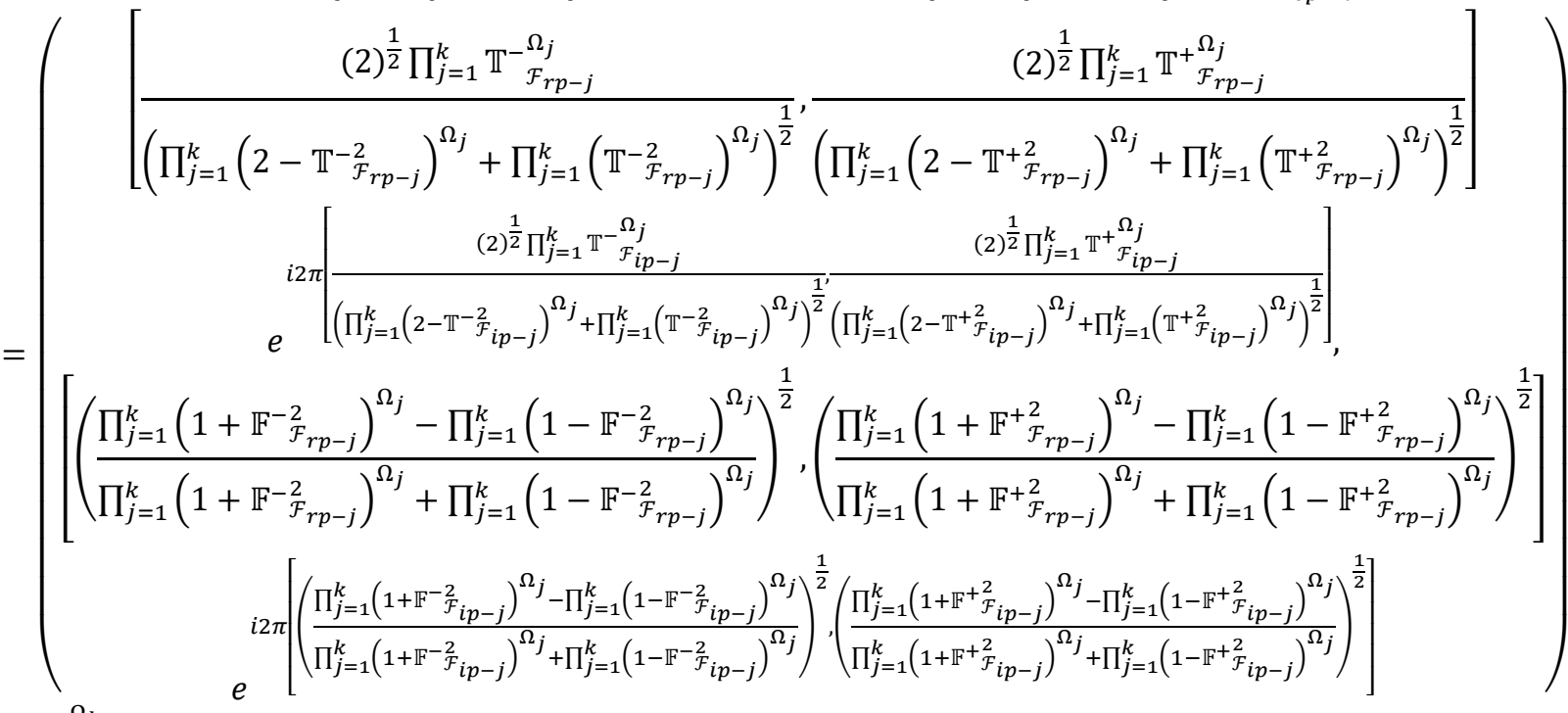

$\otimes \mathcal{F}_{c p-k+1}^{\Omega_{k+1}}$

$$
\begin{aligned}
& \left(\left[\frac{(2)^{\frac{1}{2}} \prod_{j=1}^{k+1} \mathbb{T}_{\mathcal{F}_{r p-j}}^{\Omega_{j}}}{\left(\prod_{j=1}^{k+1}\left(2-\mathbb{T}^{-2}{ }_{\mathcal{F}_{r p-j}}\right)^{\Omega_{j}}+\prod_{j=1}^{k+1}\left(\mathbb{T}^{-\frac{2}{\mathcal{F}_{r p-j}}}\right)^{\Omega_{j}}\right)^{\frac{1}{2}}}, \frac{(2)^{\frac{1}{2}} \prod_{j=1}^{k+1} \mathbb{T}_{\mathcal{F}_{r p-j}^{+\Omega_{j}}}}{\left(\prod_{j=1}^{k+!}\left(2-\mathbb{T}^{+}{ }_{\mathcal{F}_{r p-j}}^{2}\right)^{\Omega_{j}}+\prod_{j=1}^{k+!}\left(\mathbb{T}_{\mathcal{F}_{r p-j}}^{+2}\right)^{\Omega_{j}}\right)^{\frac{1}{2}}}\right]\right.
\end{aligned}
$$

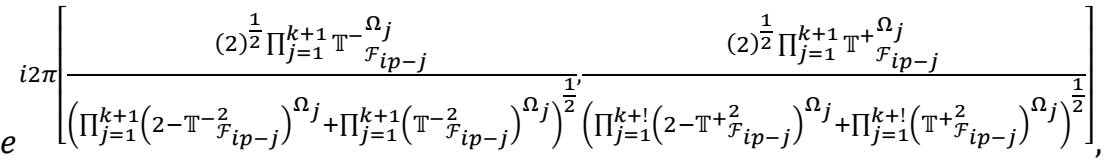

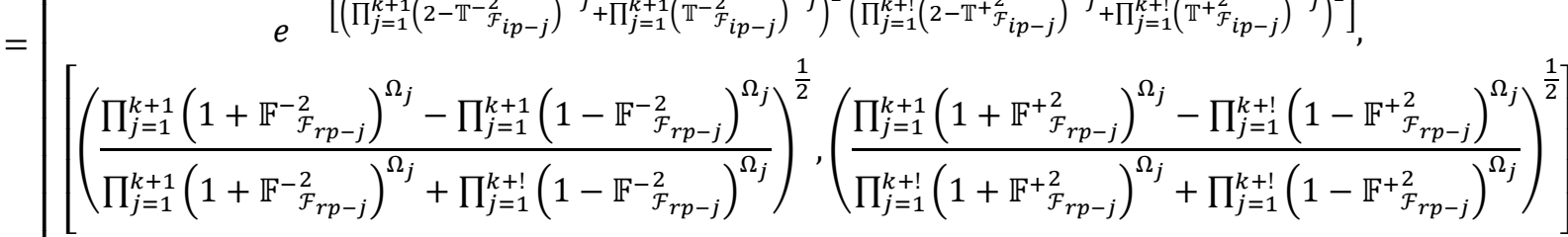

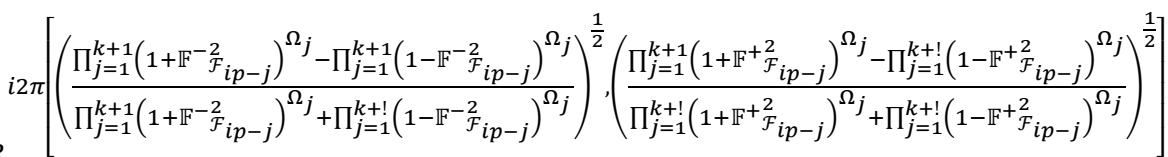

Therefore, Eq. (22) is also true for all values of $\mathbb{m}_{l e}$. 
Further, by using the information in Eq. (22), we discussed some properties for elaborated operators such as idempotency, boundedness, and monotonicity.

Property 1: Let $\mathcal{F}_{c p-j}=\left(\left[\mathbb{T}_{\mathcal{F}_{r p-j}}^{-}, \mathbb{T}_{\mathcal{F}_{r p-j}}^{+}\right] e^{i 2 \pi\left(\left[\mathbb{T}_{\overline{\mathcal{F}}_{i p-j}}^{-}, \mathbb{T}_{\mathcal{F}_{i p-j}}^{+}\right]\right)},\left[\mathbb{F}_{\mathcal{F}_{r p-j}}^{-}, \mathbb{F}_{\mathcal{F}_{r p-j}}^{+}\right] e^{i 2 \pi\left(\left[\mathbb{F}_{\overline{\mathcal{F}}_{i p-j}}^{-} \mathbb{F}_{\mathcal{F}_{i p-j}}^{+}\right]\right)}\right), j=$

$1,2, \ldots, \mathbb{m}_{l e}$ be a collection of CIVPFNs weight vector $\Omega=\left\{\Omega_{1}, \Omega_{2}, \ldots, \Omega_{\mathbb{m}_{l e}}\right\}$ with a rule that is $\sum_{j=1}^{\mathbb{m}_{l e}} \Omega_{j}=1$.

If $\mathcal{F}_{c p-j}=\mathcal{F}_{c p}=\left(\left[\mathbb{T}_{\mathcal{F}_{r p}}^{-}, \mathbb{T}_{\mathcal{F}_{r p}}^{+}\right] e^{i 2 \pi\left(\left[\mathbb{T}_{\mathcal{F}_{i p}}^{-}, \mathbb{T}_{\mathcal{F}_{i p}}^{+}\right]\right)},\left[\mathbb{F}_{\mathcal{F}_{r p}}^{-}, \mathbb{F}_{\mathcal{F}_{r p}}^{+}\right] e^{i 2 \pi\left(\left[\mathbb{F}_{\mathcal{F}_{i p}}^{-}, \mathbb{F}_{\mathcal{F}_{i p}}^{+}\right]\right)}\right)$, then

$\operatorname{CIVPFEWG}\left(\mathcal{F}_{c p-1}, \mathcal{F}_{c p-2}, \ldots, \mathcal{F}_{c p-\mathbb{m} l e}\right)=\mathcal{F}_{c p}$

Proof: $\quad$ By $\quad$ hypothesis, $\quad$ it $\quad$ is clear that

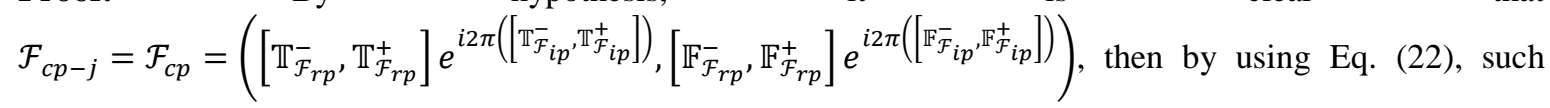
that $\operatorname{CIVPFEWG}\left(\mathcal{F}_{c p-1}, \mathcal{F}_{c p-2}, \ldots, \mathcal{F}_{c p-\mathbb{m} l e}\right)$

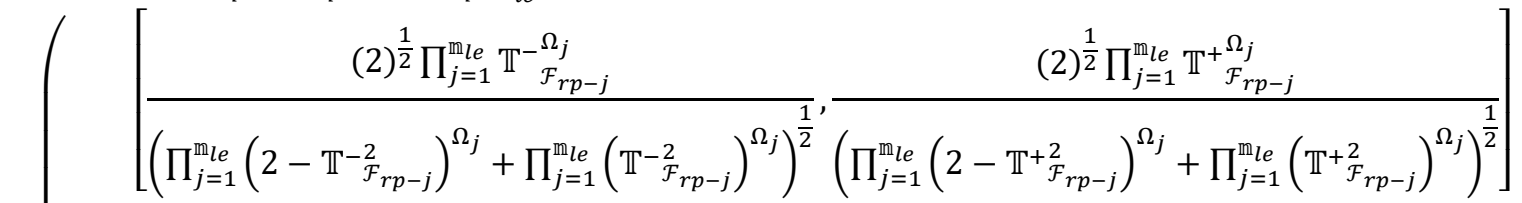

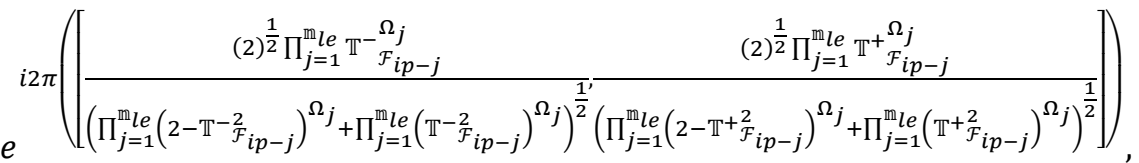

$$
\begin{aligned}
& = \\
& {\left[\left(\frac{\prod_{j=1}^{\mathbb{m} l e}\left(1+\mathbb{F}_{\mathcal{F}_{r p-j}}^{-2}\right)^{\Omega_{j}}-\prod_{j=1}^{\mathbb{m}_{l e}}\left(1-\mathbb{F}_{\mathcal{F}_{r p-j}}^{-2}\right)^{\Omega_{j}}}{\prod_{j=1}^{\mathbb{m}_{l e}}\left(1+\mathbb{F}_{\mathcal{F}_{r p-j}}^{-2}\right)^{\Omega_{j}}+\prod_{j=1}^{\mathbb{m}_{l e}}\left(1-\mathbb{F}_{\mathcal{F}_{r p-j}}^{-2}\right)^{\Omega_{j}}}\right)^{\frac{1}{2}},\left(\frac{\prod_{j=1}^{\mathbb{m}_{l e}}\left(1+\mathbb{F}_{\mathcal{F}_{r p-j}}^{2}\right)^{\Omega_{j}}-\prod_{j=1}^{\mathbb{m}_{l e}}\left(1-\mathbb{F}_{\mathcal{F}_{r p-j}}^{2}\right)^{\Omega_{j}}}{\prod_{j=1}^{\mathbb{m}_{l e}}\left(1+\mathbb{F}_{\mathcal{F}_{r p-j}}^{2}\right)^{\Omega_{j}}+\prod_{j=1}^{\mathbb{m}_{l e}}\left(1-\mathbb{F}_{\mathcal{F}_{r p-j}}^{2}\right)^{\Omega_{j}}}\right)^{\frac{1}{2}}\right]}
\end{aligned}
$$

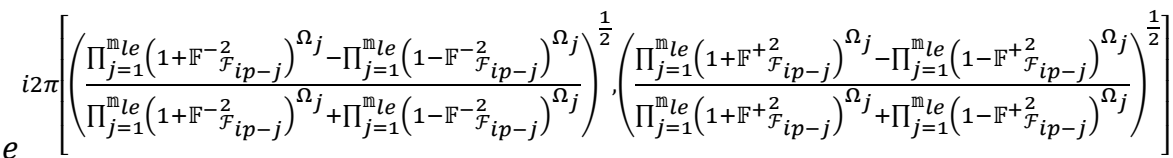

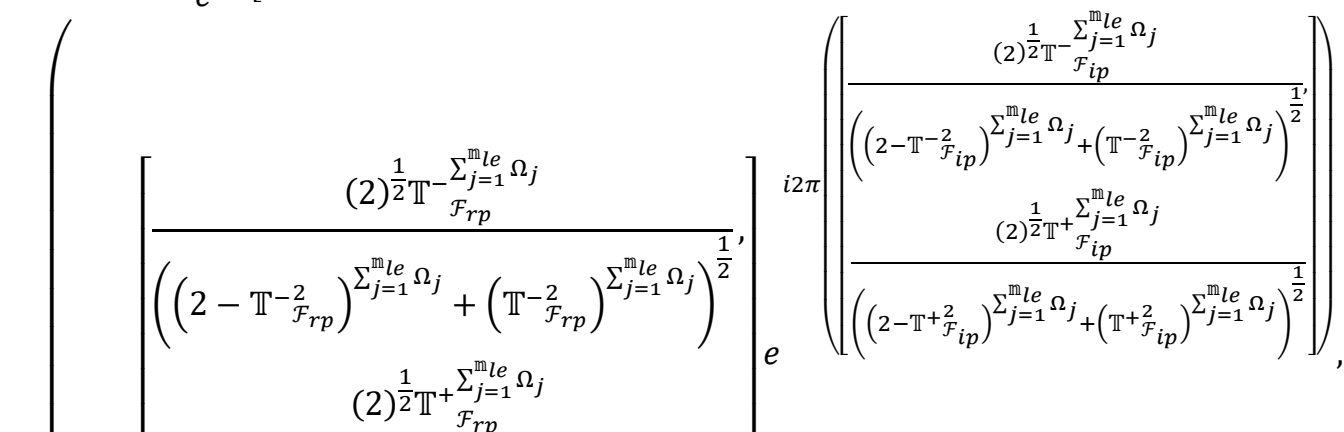

$$
\begin{aligned}
& =\left[\frac{\left(\left(2-\mathbb{T}^{+} \mathcal{F}_{r p}\right)^{\sum_{j=1}^{\mathrm{m} l e} \Omega_{j}}+\left(\mathbb{T}^{+}{ }_{\mathcal{F}_{r p}}^{2}\right)^{\sum_{j=1}^{\mathrm{m} l e} \Omega_{j}}\right)^{\frac{1}{2}}}{\left[\mathcal{F}_{r p}\right.}\right]
\end{aligned}
$$

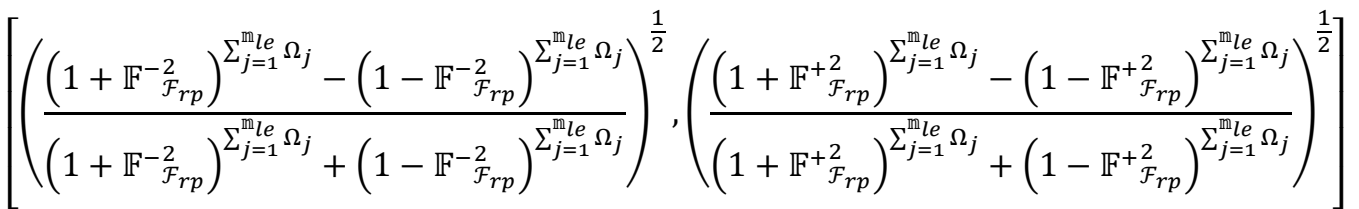

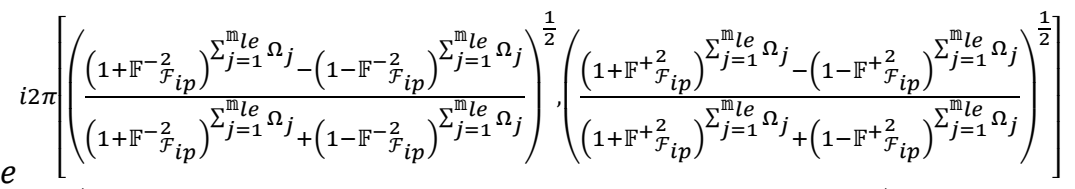

$$
\begin{aligned}
& =\left(\left[\mathbb{T}_{\mathcal{F}_{r p}}^{-}, \mathbb{T}_{\mathcal{F}_{r p}}^{+}\right] e^{i 2 \pi\left(\left[\mathbb{T}_{\mathcal{F}_{i p}}^{-}, \mathbb{T}_{\mathcal{F}_{i p}}^{+}\right]\right)},\left[\mathbb{F}_{\mathcal{F}_{r p}}^{-}, \mathbb{F}_{\mathcal{F}_{r p}}^{+}\right] e^{i 2 \pi\left(\left[\mathbb{F}_{\mathcal{F}_{i p}}^{-}, \mathbb{F}_{\mathcal{F}_{i p}}^{+}\right]\right)}\right)=\mathcal{F}_{c p}
\end{aligned}
$$




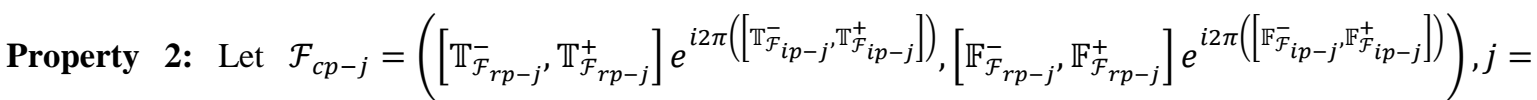
$1,2, \ldots, \mathbb{m}_{l e}$ be a collection of CIVPFNs weight vector $\Omega=\left\{\Omega_{1}, \Omega_{2}, \ldots, \Omega_{\mathbb{m}_{l e}}\right\}$ with a rule that is $\sum_{j=1}^{\mathbb{m}_{l e}} \Omega_{j}=1$. If

$\mathcal{F}_{c p}^{-}=$

$\left(\left[\min _{j} \mathbb{T}_{\mathcal{F}_{r p-j}}^{-}, \min _{j} \mathbb{T}_{\mathcal{F}_{r p-j}}^{+}\right] e^{i 2 \pi\left(\left[\min _{j} \mathbb{T}_{\mathcal{F}_{i p-j}}^{-} \min _{j} \mathbb{T}_{\mathcal{F}_{i p-j}}^{+}\right]\right)},\left[\max _{j} \mathbb{F}_{\mathcal{F}_{r p-j}}^{-}, \max _{j} \mathbb{F}_{\mathcal{F}_{r p-j}}^{+}\right] e^{i 2 \pi\left(\left[\max _{j} \mathbb{F}_{\mathcal{F}_{i p-j}}^{-} \max _{j} \mathbb{F}_{\mathcal{F}_{i p-j}}^{+}\right]\right)}\right)$ and

$\mathcal{F}_{c p}^{+}=$

$\left(\left[\max _{j} \mathbb{T}_{\mathcal{F}_{r p-j}}^{-}, \max _{j} \mathbb{T}_{\mathcal{F}_{r p-j}}^{+}\right] e^{i 2 \pi\left(\left[\max _{j} \mathbb{T}_{\mathcal{F}_{i p-j}}^{-} \max _{j} \mathbb{T}_{\mathcal{F}_{i p-j}}^{+}\right]\right)},\left[\min _{j} \mathbb{F}_{\mathcal{F}_{r p-j}}^{-}, \min _{j} \mathbb{F}_{\mathcal{F}_{r p-j}}^{+}\right] e^{i 2 \pi\left(\left[\min _{j} \mathbb{F}_{\overline{\mathcal{F}}_{i p-j}}^{-}, \min _{j} \mathbb{F}_{\mathcal{F}_{i p-j}}^{+}\right]\right)}\right)$ , then

$\mathcal{F}_{c p}^{-} \leq \operatorname{CIVPFEWG}\left(\mathcal{F}_{c p-1}, \mathcal{F}_{c p-2}, \ldots, \mathcal{F}_{c p-\mathrm{m}} l e\right) \leq \mathcal{F}_{c p}^{+}$

Proof: By hypothesis, it is clear that $\mathcal{F}_{c p}^{-}=$

$\left(\left[\min _{j} \mathbb{T}_{\mathcal{F}_{r p-j}}^{-}, \min _{j} \mathbb{T}_{\mathcal{F}_{r p-j}}^{+}\right] e^{i 2 \pi\left(\left[\min _{j} \mathbb{T}_{\mathcal{F}_{i p-j}}^{-} \min _{j} \mathbb{T}_{\mathcal{F}_{i p-j}}^{+}\right]\right)},\left[\max _{j} \mathbb{F}_{\mathcal{F}_{r p-j}}^{-}, \max _{j} \mathbb{F}_{\mathcal{F}_{r p-j}}^{+}\right] e^{i 2 \pi\left(\left[\max _{j} \mathbb{F}_{\mathcal{F}_{i p-j}}^{-}, \max _{j} \mathbb{F}_{\mathcal{F}_{i p-j}}^{+}\right]\right)}\right)$ and

$\mathcal{F}_{c p}^{+}=$

$\left(\left[\max _{j} \mathbb{T}_{\mathcal{F}_{r p-j}}^{-}, \max _{j} \mathbb{T}_{\mathcal{F}_{r p-j}}^{+}\right] e^{i 2 \pi\left(\left[\max _{j} \mathbb{T}_{\mathcal{F}_{i p-j}}^{-} \max _{j} \mathbb{T}_{\mathcal{F}_{i p-j}}^{+}\right]\right)},\left[\min _{j} \mathbb{F}_{\mathcal{F}_{r p-j}}^{-}, \min _{j} \mathbb{F}_{\mathcal{F}_{r p-j}}^{+}\right] e^{i 2 \pi\left(\left[\min _{j} \mathbb{F}_{\mathcal{F}_{i p-j}}^{-} \min _{j} \mathbb{F}_{\mathcal{F}_{i p-j}}^{+}\right]\right)}\right)$

, to prove Eq. (24), we choose that $f\left(\mathbb{x}_{e l}\right)=\frac{1-\mathbb{x}_{e l}}{1+\mathbb{x}_{e l}}$ then $f^{\prime}\left(\mathbb{x}_{e l}\right)=\frac{-2}{\left(1+\mathbb{x}_{e l}\right)^{2}}$, then it is clear that $f\left(\mathbb{x}_{e l}\right)$ is a decreasing function. Then $\mathrm{F}_{\mathcal{F}_{r p-j, \text { min }}}^{+2} \leq \mathbb{F}_{\mathcal{F}_{r p-j}}^{+2} \leq \mathbb{F}_{\mathcal{F}_{r p-j, \max }}^{2}$, further, $f\left(\mathbb{F}_{\mathcal{F}_{r p-j, m a x}}^{+2}\right) \leq f\left(\mathbb{F}_{\mathcal{F}_{r p-j}}^{2}\right) \leq$ $f\left(\mathbb{F}_{\mathcal{F}_{r p-j, \text { min }}}^{+2}\right)$, then $\frac{1-\mathbb{F}^{+}{ }_{\mathcal{F}_{r p-j, \text { max }}}^{2}}{1+\mathbb{F}^{+2}{ }_{\mathcal{F}_{r p-j, \text { max }}}^{2}} \leq \frac{1-\mathbb{F}^{+}{ }_{\mathcal{F}_{r p-j}}^{2}}{1+\mathbb{F}^{+}{ }_{\mathcal{F}_{r p-j}}^{2}} \leq \frac{1-\mathbb{F}^{+}{ }_{\mathcal{F}_{r p-j, \text { min }}}^{2}}{1+\mathbb{F}^{+}{ }_{\mathcal{F}}^{2}}$, then

Then

$$
\left(\frac{1-\mathbb{F}_{\mathcal{F}_{r p-j, \text { max }}}^{+2}}{1+\mathrm{F}_{\mathcal{F}_{r p-j, \text { max }}}^{2}}\right)^{\Omega_{j}} \leq\left(\frac{1-\mathbb{F}_{\mathcal{F}_{r p-j}}^{+2}}{1+\mathbb{F}_{\mathcal{F}_{r p-j}}^{+2}}\right)^{\Omega_{j}} \leq\left(\frac{1-\mathbb{F}_{\mathcal{F}_{r p-j, m i n}}^{2}}{1+\mathbb{F}_{\mathcal{F}_{r p-j, \min }}^{2}}\right)^{\Omega_{j}}
$$

$$
\begin{aligned}
& \prod_{j=1}^{\mathrm{m}_{l e}}\left(\frac{1-\mathrm{F}_{\mathcal{F}_{r p-j, \text { max }}}^{2}}{1+\mathrm{F}_{\mathcal{F}_{r p-j, \text { max }}}^{2}}\right)^{\Omega_{j}} \leq \prod_{j=1}^{\mathrm{m}_{l e}}\left(\frac{1-\mathrm{F}^{+}{ }_{\mathcal{F}_{r p-j}}^{2}}{1+\mathrm{F}_{\mathcal{F}_{r p-j}}^{+}}\right)^{\Omega_{j}} \leq \prod_{j=1}^{\mathrm{m}_{l e}}\left(\frac{1-\mathbb{F}_{\mathcal{F}_{r p-j, m i n}}^{+2}}{1+\mathbb{F}_{\mathcal{F}_{r p-j, m i n}}^{+2}}\right)^{\Omega_{j}} \\
& \Leftrightarrow\left(\frac{1-\mathbb{F}_{\mathcal{F}_{r p-j, \text { max }}}^{2}}{1+\mathbb{F}_{\mathcal{F}_{r p-j, \text { max }}}^{2}}\right)^{\sum_{j=1}^{\mathbb{m} l e} \Omega_{j}} \leq \prod_{j=1}^{\mathbb{m}_{l} l e}\left(\frac{1-\mathbb{F}_{\mathcal{F}_{r p-j}}^{+2}}{1+\mathbb{F}_{\mathcal{F}_{r p-j}}^{+2}}\right)^{\Omega_{j}} \leq\left(\frac{1-\mathbb{F}_{\mathcal{F}_{r p-j, \text { min }}}^{+2}}{1+\mathbb{F}_{\mathcal{F}_{r p-j, \text { min }}}^{2}}\right)^{\sum_{j=1}^{\mathbb{m} l e} \Omega_{j}} \\
& \Leftrightarrow\left(\frac{1-\mathbb{F}_{\mathcal{F}_{r p-j, \text { max }}}^{+2}}{1+\mathrm{F}_{\mathcal{F}_{r p-j, \text { max }}}^{2}}\right) \leq \prod_{j=1}^{\mathrm{m} l e}\left(\frac{1-\mathbb{F}_{\mathcal{F}_{r p-j}}^{+2}}{1+\mathbb{F}_{\mathcal{F}_{r p-j}}^{+2}}\right)^{\Omega_{j}} \leq\left(\frac{1-\mathbb{F}^{+}{ }_{\mathcal{F}_{r p-j, \text { min }}}^{2}}{1+\mathbb{F}_{\mathcal{F}_{r p-j, \text { min }}}^{+2}}\right) \\
& \Leftrightarrow\left(\frac{2}{1+\mathbb{F}^{+}{ }_{\mathcal{F}_{r p-j, \text { max }}}^{2}}\right) \leq 1+\prod_{j=1}^{\mathrm{m}_{l} l e}\left(\frac{1-\mathbb{F}^{+}{ }_{\mathcal{F}_{r p-j}}^{2}}{1+\mathbb{F}^{+}{ }_{\mathcal{F}_{r p-j}}^{2}}\right)^{\Omega_{j}} \leq\left(\frac{2}{1+\mathbb{F}^{+}{ }_{\mathcal{F}_{r p-j, \text { min }}}^{2}}\right) \\
& \Leftrightarrow \frac{1}{\left(\frac{2}{1+\mathbb{F}^{+}{ }_{\mathcal{F}_{r p-j, \text { min }}}^{2}}\right)} \leq \frac{1}{1+\prod_{j=1}^{\mathrm{m} l e}\left(\frac{1-\mathbb{F}_{\mathcal{F}_{r p-j}}^{2}}{1+\mathbb{F}^{+2}{ }_{\mathcal{F}_{r p-j}}}\right)^{\Omega_{j}}} \leq \frac{1}{\left(\frac{2}{1+\mathbb{F}^{+}{ }_{\mathcal{F}_{r p-j, \text { max }}}^{2}}\right)} \\
& \Leftrightarrow 1+\mathbb{F}_{\mathcal{F}_{r p-j, \text { min }}}^{+2} \leq \frac{2}{1+\prod_{j=1}^{\mathbb{m} l e}\left(\frac{1-\mathbb{F}_{\mathcal{F}_{r p-j}}^{2}}{1+\mathbb{F}_{\mathcal{F}_{r p-j}}^{2}}\right)^{\Omega_{j}}} \leq 1+\mathbb{F}_{\mathcal{F}_{r p-j, \text { max }}}^{+2} \\
& \Leftrightarrow \mathbb{F}_{\mathcal{F}_{r p-j, \text { min }}}^{+2} \leq \frac{2}{1+\prod_{j=1}^{\mathbb{m} l e}\left(\frac{1-\mathbb{F}_{\mathcal{F}_{r p-j}}^{+2}}{1+\mathbb{F}_{\mathcal{F}_{r p-j}}^{2}}\right)^{\Omega_{j}}} \leq \mathbb{F}_{\mathcal{F}_{r p-j, \max }^{+}}^{+2}
\end{aligned}
$$


And similarly

$$
\begin{aligned}
& \Leftrightarrow \mathbb{F}_{\mathcal{F}_{r p-j, \min }}^{+2} \leq \frac{\prod_{j=1}^{\mathrm{m} l e}\left(1+\mathbb{F}_{\mathcal{F}_{r p-j}}^{+2}\right)^{\Omega_{j}}-\prod_{j=1}^{\mathrm{m}_{l e}}\left(1-\mathbb{F}_{\mathcal{F}_{r p-j}}^{+2}\right)^{\Omega_{j}}}{\prod_{j=1}^{\mathrm{m}_{l e}}\left(1+\mathbb{F}_{\mathcal{F}_{r p-j}}^{+2}\right)^{\Omega_{j}}+\prod_{j=1}^{\mathrm{m}_{l e}}\left(1-\mathrm{F}_{\mathcal{F}_{r p-j}}^{+2}\right)^{\Omega_{j}}} \leq \mathbb{F}_{\mathcal{F}_{r p-j, \max }}^{+^{2}} \\
& \Leftrightarrow \mathbb{F}^{+}{ }_{\mathcal{F}_{r p-j, \text { min }}} \leq\left(\frac{\prod_{j=1}^{\mathbb{m} l e}\left(1+\mathbb{F}_{\mathcal{F}_{r p-j}}^{+2}\right)^{\Omega_{j}}-\prod_{j=1}^{\mathrm{m} l e}\left(1-\mathbb{F}_{\mathcal{F}_{r p-j}}^{+2}\right)^{\Omega_{j}}}{\prod_{j=1}^{\mathbb{m} l e}\left(1+\mathbb{F}^{+}{ }_{\mathcal{F}_{r p-j}}^{2}\right)^{\Omega_{j}}+\prod_{j=1}^{\mathbb{m} l e}\left(1-\mathbb{F}_{\mathcal{F}_{r p-j}}^{+2}\right)^{\Omega_{j}}}\right)^{\frac{1}{2}} \leq \mathbb{F}^{+}{ }_{\mathcal{F}_{r p-j, \text { max }}}
\end{aligned}
$$

$$
\mathbb{F}_{\mathcal{F}_{r p-j, \text { min }}} \leq\left(\frac{\prod_{j=1}^{\mathbb{m} l e}\left(1+\mathbb{F}_{\mathcal{F}_{r p-j}}^{-2}\right)^{\Omega_{j}}-\prod_{j=1}^{\mathbb{m}_{l e}}\left(1-\mathbb{F}_{\mathcal{F}_{r p-j}}^{-2}\right)^{\Omega_{j}}}{\prod_{j=1}^{\mathbb{m} l e}\left(1+\mathbb{F}_{\mathcal{F}_{r p-j}}^{-2}\right)^{\Omega_{j}}+\prod_{j=1}^{\mathbb{m}_{l e}}\left(1-\mathbb{F}_{\mathcal{F}_{r p-j}}^{-2}\right)^{\Omega_{j}}}\right)^{\frac{1}{2}} \leq \mathbb{F}_{\mathcal{F}_{r p-j, \text { max }}}
$$

Similarly, we will determine the imaginary part of the FG, such that

$$
\begin{aligned}
& \mathbb{F}^{+}{ }_{\mathcal{F}_{i p-j, \text { min }}} \leq\left(\frac{\prod_{j=1}^{\mathrm{m} l e}\left(1+\mathrm{F}_{\mathcal{F}_{i p-j}}^{+2}\right)^{\Omega_{j}}-\prod_{j=1}^{\mathrm{m} l e}\left(1-\mathbb{F}_{\mathcal{F}_{i p-j}}^{+2}\right)^{\Omega_{j}}}{\prod_{j=1}^{\mathrm{m} l e}\left(1+\mathrm{F}^{+}{ }_{\mathcal{F}_{i p-j}}^{2}\right)^{\Omega_{j}}+\prod_{j=1}^{\mathrm{m}_{l e}}\left(1-\mathbb{F}_{\mathcal{F}_{i p-j}}^{+2}\right)^{\Omega_{j}}}\right)^{\frac{1}{2}} \leq \mathbb{F}^{+}{ }_{\mathcal{F}_{i p-j, \text { max }}} \\
& \mathbb{F}_{\mathcal{F}_{i p-j, \text { min }}} \leq\left(\frac{\prod_{j=1}^{\mathbb{m}_{l e}}\left(1+\mathbb{F}_{\mathcal{F}_{i p-j}}^{-2}\right)^{\Omega_{j}}-\prod_{j=1}^{\mathbb{m}_{l e}}\left(1-\mathbb{F}_{\mathcal{F}_{i p-j}}^{-2}\right)^{\Omega_{j}}}{\prod_{j=1}^{\mathrm{m} l e}\left(1+\mathbb{F}_{\mathcal{F}_{i p-j}}^{-2}\right)^{\Omega_{j}}+\prod_{j=1}^{\mathbb{m}_{l e}}\left(1-\mathbb{F}_{\mathcal{F}_{i p-j}}^{-2}\right)^{\Omega_{j}}}\right)^{\frac{1}{2}} \leq \mathbb{F}_{\mathcal{F}_{i p-j, \text { max }}}
\end{aligned}
$$

Similarly, by using another decreasing function $g\left(y_{e l}\right)=\frac{2-y_{e l}}{y_{e l}}$ then $g^{\prime}\left(y_{e l}\right)=\frac{-2}{y_{e l}^{2}}$, then we will find the value of real and unreal parts of the TG, such that

And

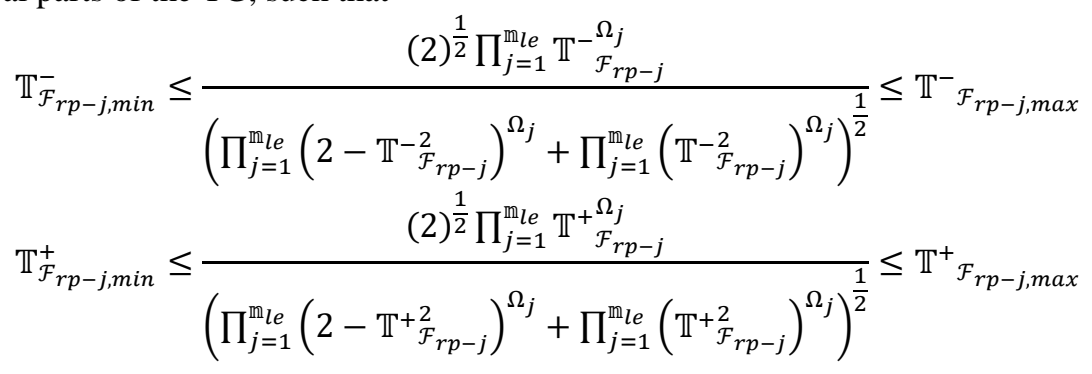

$$
\begin{aligned}
& \mathbb{T}_{\mathcal{F}_{i p-j, \text { min }}}^{-} \leq \frac{(2)^{\frac{1}{2}} \prod_{j=1}^{\mathbb{m} l e} \mathbb{T}^{-\Omega_{j}} \mathcal{F}_{i p-j}}{\left(\prod_{j=1}^{\mathbb{m} l e}\left(2-\mathbb{T}_{\mathcal{F}_{i p-j}}^{-2}\right)^{\Omega_{j}}+\prod_{j=1}^{\mathbb{M}_{l e}}\left(\mathbb{T}^{-2} \mathcal{F}_{i p-j}\right)^{\Omega_{j}}\right)^{\frac{1}{2}}} \leq \mathbb{T}^{-} \mathcal{F}_{i p-j, \text { max }} \\
& \mathbb{T}_{\mathcal{F}_{i p-j, \text { min }}}^{+} \leq \frac{(2)^{\frac{1}{2}} \prod_{j=1}^{\mathrm{m} l e} \mathbb{T}^{+}{ }_{\mathcal{F}_{i p-j}}^{\Omega_{j}}}{\left(\prod_{j=1}^{\mathbb{m} l e}\left(2-\mathbb{T}^{+}{ }_{\mathcal{F}_{i p-j}}^{2}\right)^{\Omega_{j}}+\prod_{j=1}^{\mathbb{m} l e}\left(\mathbb{T}^{+}{ }_{\mathcal{F}_{i p-j}}^{2}\right)^{\Omega_{j}}\right)^{\frac{1}{2}}} \leq \mathbb{T}^{+}{ }_{\mathcal{F}_{i p-j, \text { max }}}
\end{aligned}
$$

Then, by using Eq. (13) and Eq. (14), we easily determine the required result, such that

$$
\mathcal{F}_{c p}^{-} \leq \operatorname{CIVPFEWG}\left(\mathcal{F}_{c p-1}, \mathcal{F}_{c p-2}, \ldots, \mathcal{F}_{c p-\mathbb{m}_{l e}}\right) \leq \mathcal{F}_{c p}^{+}
$$

Property 3: Let $\mathcal{F}_{c p-j}=\left(\left[\mathbb{T}_{\mathcal{F}_{r p-j}}^{-}, \mathbb{T}_{\mathcal{F}_{r p-j}}^{+}\right] e^{i 2 \pi\left(\left[\mathbb{T}_{\mathcal{F}_{i p-j}}^{-}, \mathbb{T}_{\mathcal{F}_{i p-j}}^{+}\right]\right)},\left[\mathbb{F}_{\mathcal{F}_{r p-j}}^{-}, \mathbb{F}_{\mathcal{F}_{r p-j}}^{+}\right] e^{i 2 \pi\left(\left[\mathbb{F}_{\mathcal{F}_{i p-j}}^{-}, \mathbb{F}_{\mathcal{F}_{i p-j}}^{+}\right]\right)}\right), j=$ $1,2, \ldots, \mathbb{m}_{l e}$ be a collection of CIVPFNs weight vector $\Omega=\left\{\Omega_{1}, \Omega_{2}, \ldots, \Omega_{\mathbb{m}_{l e}}\right\}$ with a rule that is $\sum_{j=1}^{\mathbb{m}_{l e}} \Omega_{j}=1$. If, $\mathcal{F}_{c p-j} \leq \mathcal{F}_{c p-j}^{*}$ then

$\operatorname{CIVPFEWG}\left(\mathcal{F}_{c p-1}, \mathcal{F}_{c p-2}, \ldots, \mathcal{F}_{c p-\mathbb{m}_{l e}}\right) \leq \operatorname{CIVPFEWG}\left(\mathcal{F}_{c p-1}^{*}, \mathcal{F}_{c p-2}^{*}, \ldots, \mathcal{F}_{c p-\mathbb{m}_{l e}}^{*}\right)$

Proof: Straightforward

Definition 5: Let $\mathcal{F}_{c p-j}=\left(\left[\mathbb{T}_{\mathcal{F}_{r p-j}}^{-}, \mathbb{T}_{\mathcal{F}_{r p-j}}^{+}\right] e^{i 2 \pi\left(\left[\mathbb{T}_{\mathcal{F}_{i p-j}}^{-}, \mathbb{T}_{\mathcal{F}_{i p-j}}^{+}\right]\right)},\left[\mathbb{F}_{\mathcal{F}_{r p-j}}^{-}, \mathbb{F}_{\mathcal{F}_{r p-j}}^{+}\right] e^{i 2 \pi\left(\left[\mathbb{F}_{\mathcal{F}_{i p-j}}^{-} \mathbb{F}_{\mathcal{F}_{i p-j}}^{+}\right]\right)}\right), j=$ $1,2, \ldots, \mathbb{m}_{l e}$ be a collection of CIVPFNs weight vector $\Omega=\left\{\Omega_{1}, \Omega_{2}, \ldots, \Omega_{\mathbb{m}_{l e}}\right\}$ with a rule that is $\sum_{j=1}^{\mathbb{m}_{l e}} \Omega_{j}=1$. Then the CIVPFEOWG operator is initiated by: 
$\operatorname{CIVPFEOWG}\left(\mathcal{F}_{c p-1}, \mathcal{F}_{c p-2}, \ldots, \mathcal{F}_{c p-\mathrm{m}_{l e}}\right)=\bigotimes_{j=1}^{\mathrm{m}_{l}}\left(\mathcal{F}_{c p-\mathrm{O}(j)}^{\Omega_{j}}\right)$

Where $\mathrm{O}(j)$ is expressed the permutation of $\left(j=1,2, \ldots, \mathrm{m}_{l e}\right)$ with a rule that is $\mathrm{O}(j) \leq \mathrm{O}(j-1)$. If we fixed the value of $\Omega_{j}=\frac{1}{\mathrm{~m}_{l e}}$ for all $j$, then the CIVPFEOWG operator is converted for complex interval-valued Pythagorean fuzzy ordered geometric (CIVPFOG) operator such that

$\operatorname{CIVPFOG}\left(\mathcal{F}_{c p-1}, \mathcal{F}_{c p-2}, \ldots, \mathcal{F}_{c p-\mathbb{m}_{l e}}\right)=\left(\otimes_{j=1}^{\mathbb{m}_{l e}}\left(\mathcal{F}_{c p-\mathrm{O}(j)}\right)\right)^{\frac{1}{\mathrm{~m}_{l} e}}$

Theorem 3: Let $\mathcal{F}_{c p-j}=\left(\left[\mathbb{T}_{\mathcal{F}_{r p-j}}^{-}, \mathbb{T}_{\mathcal{F}_{r p-j}}^{+}\right] e^{i 2 \pi\left(\left[\mathbb{T}_{\mathcal{F}_{i p-j}}^{-} \mathbb{T}^{+} \mathbb{F}_{i p-j}\right]\right)},\left[\mathbb{F}_{\mathcal{F}_{r p-j}}^{-}, \mathbb{F}_{\mathcal{F}_{r p-j}}^{+}\right] e^{i 2 \pi\left(\left[\mathbb{F}_{\overline{\mathcal{F}}_{i p-j}} \mathbb{F}_{\mathcal{F}_{i p-j}}^{+}\right]\right)}\right), j=$

$1,2, \ldots, \mathbb{m}_{l e}$ be a collection of CIVPFNs weight vector $\Omega=\left\{\Omega_{1}, \Omega_{2}, \ldots, \Omega_{\mathbb{m}_{l e}}\right\}$ with a rule that is $\sum_{j=1}^{\mathbb{m}_{l} e} \Omega_{j}=1$.

Then by using Eq. (26), we determine

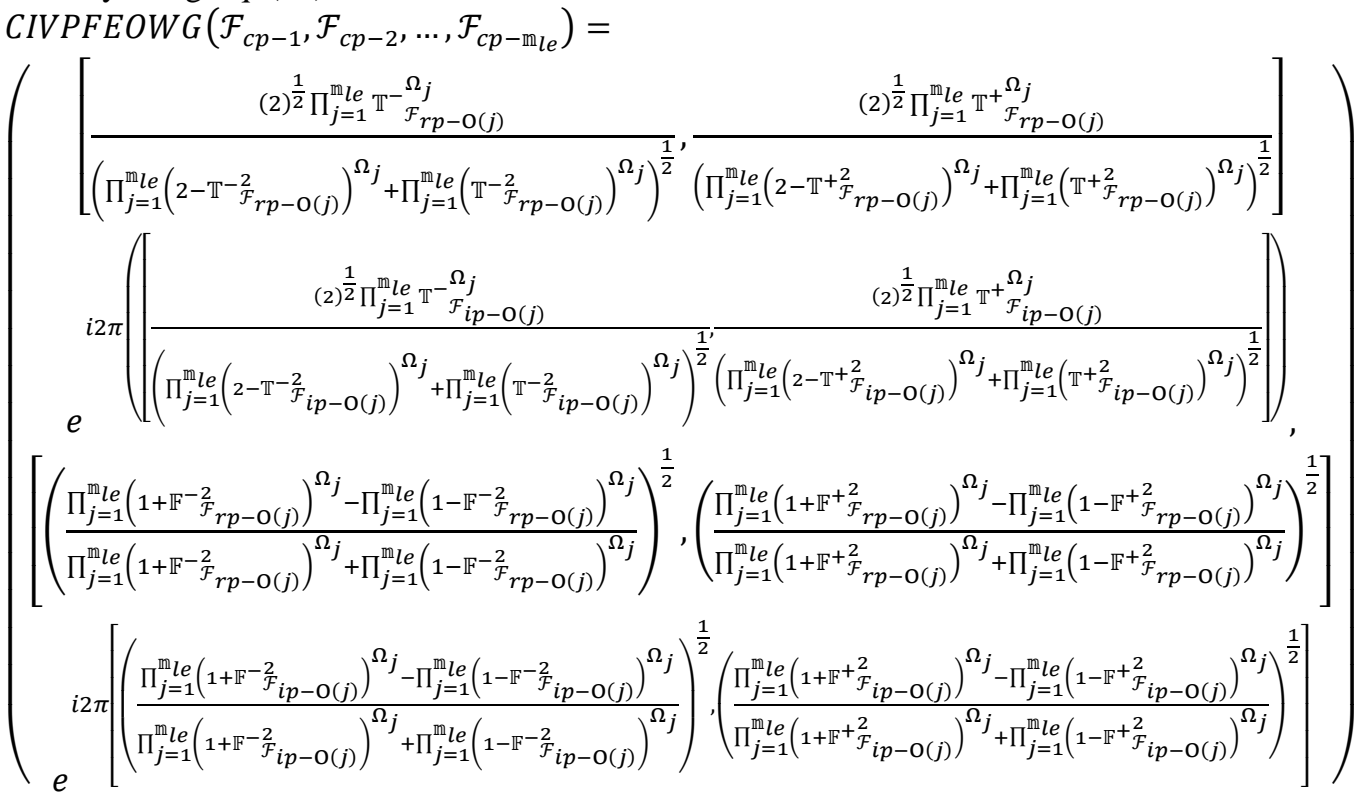

Proof: Straightforward.

Further, by using the information in Eq. (28), we discussed some properties for elaborated operators such as idempotency, boundedness, and monotonicity.

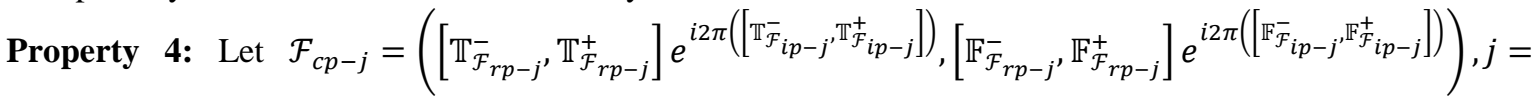
$1,2, \ldots, \mathbb{m}_{l e}$ be a collection of CIVPFNs weight vector $\Omega=\left\{\Omega_{1}, \Omega_{2}, \ldots, \Omega_{\mathbb{m}_{l}}\right\}$ with a rule that is $\sum_{j=1}^{\mathbb{m} l e} \Omega_{j}=1$.

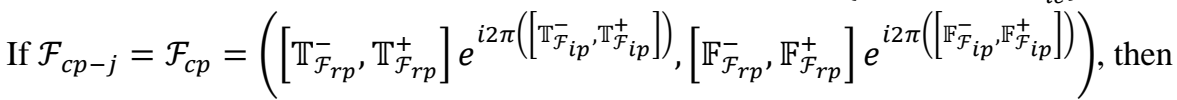

$\operatorname{CIVPFEOWG}\left(\mathcal{F}_{c p-1}, \mathcal{F}_{c p-2}, \ldots, \mathcal{F}_{c p-\mathrm{m}_{l e}}\right)=\mathcal{F}_{c p}$

Proof: Straightforward.

Property 5: Let $\mathcal{F}_{c p-j}=\left(\left[\mathbb{T}_{\mathcal{F}_{r p-j}}^{-}, \mathbb{T}_{\mathcal{F}_{r p-j}}^{+}\right] e^{i 2 \pi\left(\left[\mathbb{T}_{\mathcal{F}_{i p-j}}^{-} \mathbb{T}^{+} \mathbb{F}_{i p-j}\right]\right.},\left[\mathbb{F}_{\mathcal{F}_{r p-j}}, \mathbb{F}_{\mathcal{F}_{r p-j}}^{+}\right] e^{i 2 \pi\left(\left[\mathbb{F}_{\overline{\mathcal{F}}_{i p-j}} \mathbb{F}_{\mathcal{F}_{i p-j}}^{+}\right]\right.}\right), j=$ $1,2, \ldots, \mathbb{m}_{l e}$ be a collection of CIVPFNs weight vector $\Omega=\left\{\Omega_{1}, \Omega_{2}, \ldots, \Omega_{\mathbb{m}_{l e}}\right\}$ with a rule that is $\sum_{j=1}^{\mathbb{m}_{l} e} \Omega_{j}=1$. If

$\mathcal{F}_{c p}^{-}=$

$\left(\left[\min _{j} \mathbb{T}_{\mathcal{F}_{r p-j}}^{-}, \min _{j} \mathbb{T}_{\mathcal{F}_{r p-j}}^{+}\right] e^{i 2 \pi\left(\left[\min _{j} \mathbb{T}_{\mathcal{F}_{i p-j}}^{-} \min _{j} \mathbb{T}_{\mathcal{F}_{i p-j}}^{+}\right]\right)},\left[\max _{j} \mathbb{F}_{\mathcal{F}_{r p-j}}^{-}, \max _{j} \mathbb{F}_{\mathcal{F}_{r p-j}}^{+}\right] e^{i 2 \pi\left(\left[\max _{j} \mathbb{F}_{\overline{\mathcal{F}}_{i p-j}}^{-\max _{j}} \mathbb{F}_{\mathcal{F}_{i p-j}}^{+}\right]\right)}\right)$ and

$\mathcal{F}_{c p}^{+}=$

$\left(\left[\max _{j} \mathbb{T}_{\mathcal{F}_{r p-j}}^{-}, \max _{j} \mathbb{T}_{\mathcal{F}_{r p-j}}^{+}\right] e^{i 2 \pi\left(\left[\max _{j} \mathbb{T}_{\mathcal{F}_{i p-j}}^{-} \max _{j} \mathbb{T}_{\mathcal{F}_{i p-j}}^{+}\right]\right)},\left[\min _{j} \mathbb{F}_{\mathcal{F}_{r p-j}}^{-}, \min _{j} \mathbb{F}_{\mathcal{F}_{r p-j}}^{+}\right] e^{i 2 \pi\left(\left[\min _{j} \mathbb{F}_{\mathcal{F}_{i p-j}}^{-} \min _{j} \mathbb{F}_{\mathcal{F}_{i p-j}}^{+}\right]\right)}\right)$ , then

$\mathcal{F}_{c p}^{-} \leq \operatorname{CIVPFEOWG}\left(\mathcal{F}_{c p-1}, \mathcal{F}_{c p-2}, \ldots, \mathcal{F}_{c p-\mathrm{m}_{l e}}\right) \leq \mathcal{F}_{c p}^{+}$

Proof: Straightforward. 


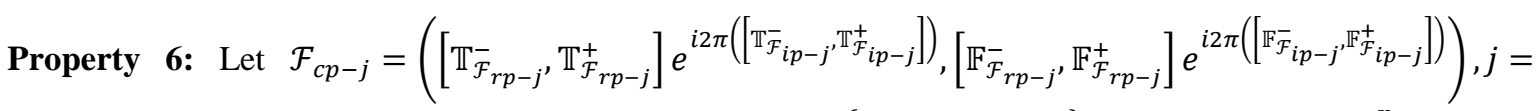
$1,2, \ldots, \mathbb{m}_{l e}$ be a collection of CIVPFNs weight vector $\Omega=\left\{\Omega_{1}, \Omega_{2}, \ldots, \Omega_{\mathbb{m}_{l e}}\right\}$ with a rule that is $\sum_{j=1}^{\mathbb{m}_{l e}} \Omega_{j}=1$. If, $\mathcal{F}_{c p-j} \leq \mathcal{F}_{c p-j}^{*}$ then

$\operatorname{CIVPFEOWG}\left(\mathcal{F}_{c p-1}, \mathcal{F}_{c p-2}, \ldots, \mathcal{F}_{c p-\mathbb{m}_{l e}}\right) \leq \operatorname{CIVPFEOWG}\left(\mathcal{F}_{c p-1}^{*}, \mathcal{F}_{c p-2}^{*}, \ldots, \mathcal{F}_{c p-\mathrm{m}_{l e}}^{*}\right)$

Proof: Straightforward.

\section{MCDM based on Proposed Operators.}

Certain individuals have utilized the theory of aggregation operators, measures, and methods in the environment of IFSs, PFSs, IVIFSs, IVPFSs, CIFSs, CIVIFSs, CPFSs, and their application in the MCDM technique to determine the consistency and dominance of the prevailing works. But up to date, no one proposed the theory of CIVPFSs by using any kind of operators and their application in the MCDM technique. The goal of this manuscript is to utilize the Einstein geometric aggregation operators based on CIVPFSs to determine the dominance of the elaborated works. For this, we choose the group of alternatives $\mathcal{F}_{A l-1}, \mathcal{F}_{A l-2}, \ldots, \mathcal{F}_{A l-n}$ and their attributes $\breve{\mathcal{F}}_{A t-1}, \breve{\mathcal{F}}_{A t-2}, \ldots, \breve{\mathcal{F}}_{A t-m}$ concerning weight vector $\Omega=$ $\left\{\Omega_{1}, \Omega_{2}, \ldots, \Omega_{\mathbb{m}_{l}}\right\}$ with a rule that is $\sum_{j=1}^{\mathbb{m}} l e \Omega_{j}=1$. To evaluate the above issues, we construct the decision matrix whose very items are in the form of complex interval-valued Pythagorean fuzzy numbers such that

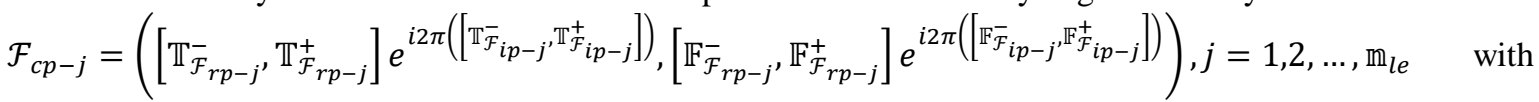
some rules that are $0 \leq \mathbb{T}_{\mathcal{F}_{r p}}^{+}{ }^{2}\left(\mathbb{x}_{e l}\right)+\mathbb{F}_{\mathcal{F}_{r p}}^{+}{ }^{2}\left(\mathbb{x}_{e l}\right) \leq 1,0 \leq \mathbb{T}_{\mathcal{F}_{i p}}^{+}{ }^{2}\left(\mathbb{x}_{e l}\right)+\mathbb{F}_{\mathcal{F}_{i p}}^{+}{ }^{2}\left(\mathbb{x}_{e l}\right) \leq 1$. Then by using the above family of $n$ alternatives and $m$ attributes, we developed the algorithm whose steps are as follows:

Step 1: Develop the decision matrix, whose every item in the form of CIVPFNs.

Step 2: By using the CIVPFEWG operator to aggregate the entries of the decision matrix.

Step 3: By using the score function, we find the Score values of the aggregated values of Step 2.

Step 5: Rank all alternatives and examine the best one.

As shown above, we illustrate certain numerical examples to determine the consistency and validity of the elaborated operators.

Example: With the quick advancement of monetary globalization, and the developing undertaking rivalry climate, the rivalry between current ventures has become the contest between supply chains. The variety of individuals burning through is expanding, and the new item life cycles are getting more limited. The instability of the interest market and from outer elements drives endeavors for viable inventory network mix and the board, just as essential partnerships with different ventures to improve the center intensity and oppose outside hazard. The critical measure to accomplishing this objective is provider choice. Hence, the provider choice issue has acquired a great deal of consideration, regardless of whether in respect to inventory network the executive's hypothesis or real creation the board issues. To delineate our proposed technique in this article, we give a mathematical guide to choosing green providers in the green inventory network the executives utilizing CIVPFNs. For this, we choose the family of five possible green suppliers in green supplier chain management $\mathcal{F}_{A l-1}, \mathcal{F}_{A l-2}, \mathcal{F}_{A l-3}, \mathcal{F}_{A l-4}, \mathcal{F}_{A l-5}$ and their attributes are in the form of selection experts whose expressions are followed as:

$\mathcal{F}_{A t-1}$ : Expressed the product quality factor.

$\mathcal{F}_{A t-2}$ : Expressed the environmental factor.

$\mathcal{F}_{A t-3}$ : Expressed the delivery factor.

$\mathcal{F}_{A t-4}$ : Expressed price factor.

To determine the above troubles, we choose the family of weight vectors such that $0.3,0.2,0.3,0.2$, Then by using the above family of $n$ alternatives and $m$ attributes, we developed the algorithm whose steps are as follows:

Step 1: We developed the decision matrix, whose every item in the form of CIVPFNs is discussed in the shape of Table 1. 
Table 1. Original decision matrix covers the complex interval-valued Pythagorean fuzzy numbers.

\begin{tabular}{|c|c|c|c|c|}
\hline & $\mathcal{F}_{A t-1}$ & $\mathcal{F}_{A t-2}$ & $\mathcal{F}_{A t-3}$ & $\mathcal{F}_{A t-4}$ \\
\hline \multirow[t]{2}{*}{$\mathcal{F}_{A l-1}$} & $0.4,0.5] e^{i 2 \pi([\bar{C}}$ & {$[0.41,0.51] e^{i 2 \pi}$} & $\left([0.42,0.52] e^{i 2 \pi([0.03,0.22])}\right.$, & {$[0.43,0.53] e^{i 2 \pi(}$} \\
\hline & & & $2] e^{i 2 \pi([0.12,0.22])}$ & \\
\hline \multirow[t]{2}{*}{$\mathcal{F}_{A l-2}$} & $([0$ & $61] e^{i 2 \pi([0.03,0.31])}$, & $\left([0.52,0.62] e^{i 2 \pi([0.04,0.32])}\right.$, & $([0.5$ \\
\hline & & {$[0.2$} & {$[0.2$} & \\
\hline \multirow{2}{*}{$\mathcal{F}_{A l-3}$} & $([0.4$ & $e^{i 2 \pi([0.02,0.51])}$, & $\left([0.42,0.62] e^{i 2 \pi([0.03, \mathrm{C}}\right.$ & $(0.4$ \\
\hline & {$[0$} & {$[0$.} & {$[0$} & \\
\hline \multirow[t]{2}{*}{$\mathcal{F}_{A l-4}$} & $([0$. & ]$e^{i 2 \pi([0.04,0.51])}$, & $.32] e^{i 2 \pi([0.0}$ & $([0.1$ \\
\hline & & {$[0$} & {$[0$} & \\
\hline \multirow[t]{2}{*}{$\mathcal{F}_{A l-5}$} & $\left([0.3,0.7] e^{i 2 \pi([0.03,0.7])}\right.$ & $\left([0.31,0.71] e^{i 2 \pi([}\right.$ & $\left([0.32,0.72] e^{i 2 \pi}\right.$ & $\left([0.33,0.73] e^{i 2 \pi}\right.$ \\
\hline & {$[0.1,0.2] e^{i 2 \pi([0.1,0.2])}$} & {$[0.11,0.21] e^{i 2 \pi([0.11,0.21])}$} & {$[0.12,0.22] e^{i 2 \pi([0.12,0.22])}$} & {$[0.13,0.23] e$} \\
\hline
\end{tabular}

Step 2: By using the CIVPFEWG operator to aggregate the entries of the decision matrix are discussed below.

$$
\begin{gathered}
\mathcal{F}_{A l-1}=\operatorname{CIVPFEWG}\left(\mathcal{F}_{A t-1}, \mathcal{F}_{A t-2}, \mathcal{F}_{A t-3}, \mathcal{F}_{A t-4}\right)=\left(\begin{array}{c}
{[0.4139,0.5139] e^{i 2 \pi([0.0211,0.2137])},} \\
\left.[0.1145,0.1145] e^{i 2 \pi([0.1145,0.2143])}\right)
\end{array}\right) \\
\mathcal{F}_{A l-2}=\operatorname{CIVPFEWG}\left(\mathcal{F}_{A t-1}, \mathcal{F}_{A t-2}, \mathcal{F}_{A t-3}, \mathcal{F}_{A t-4}\right)=\left(\begin{array}{l}
{[0.5139,0.6139] e^{i 2 \pi([0.0321,0.3138])},} \\
\left.[0.2143,0.3142] e^{i 2 \pi([0.1145,0.3142])}\right)
\end{array}\right) \\
\mathcal{F}_{A l-3}=\operatorname{CIVPFEWG}\left(\mathcal{F}_{A t-1}, \mathcal{F}_{A t-2}, \mathcal{F}_{A t-3}, \mathcal{F}_{A t-4}\right)=\left(\begin{array}{c}
{[0.4139,0.6139] e^{i 2 \pi([0.0211,0.5139])},} \\
\left.[0.1145,0.2143] e^{i 2 \pi([0.1145,0.2143])}\right)
\end{array}\right) \\
\mathcal{F}_{A l-4}=\operatorname{CIVPFEWG}\left(\mathcal{F}_{A t-1}, \mathcal{F}_{A t-2}, \mathcal{F}_{A t-3}, \mathcal{F}_{A t-4}\right)=\left(\begin{array}{c}
{[0.1135,0.3138] e^{i 2 \pi([0.0425,0.5139])},} \\
\left.[0.1145,0.2143] e^{i 2 \pi([0.2143,0.3142])}\right)
\end{array}\right) \\
\mathcal{F}_{A l-5}=\operatorname{CIVPFEWG}\left(\mathcal{F}_{A t-1}, \mathcal{F}_{A t-2}, \mathcal{F}_{A t-3}, \mathcal{F}_{A t-4}\right)=\left(\begin{array}{c}
{[0.3138,0.714] e^{i 2 \pi([0.0425,0.714])},} \\
\left.[0.1145,0.2143] e^{i 2 \pi([0.1145,0.2143])}\right)
\end{array}\right)
\end{gathered}
$$

Step 3: By using the score function, we find the Score values of the aggregated values of Step 2, which are discussed below.

$$
\begin{gathered}
\mathbb{S}^{s f}\left(\mathcal{F}_{A l-1}\right)=0.1981, \mathbb{S}^{s f}\left(\mathcal{F}_{A l-2}\right)=0.2420, \mathbb{S}^{s f}\left(\mathcal{F}_{A l-3}\right)=0.3473, \mathbb{S}^{s f}\left(\mathcal{F}_{A l-4}\right)=0.0868, \mathbb{S}^{s f}\left(\mathcal{F}_{A l-5}\right) \\
=0.5009
\end{gathered}
$$

Step 5: By using the above Score values, we rank all alternatives and examine the best one such that

That means that

$$
\mathbb{S}^{s f}\left(\mathcal{F}_{A l-5}\right) \geq \mathbb{S}^{s f}\left(\mathcal{F}_{A l-3}\right) \geq \mathbb{S}^{s f}\left(\mathcal{F}_{A l-2}\right) \geq \mathbb{S}^{s f}\left(\mathcal{F}_{A l-1}\right) \geq \mathbb{S}^{s f}\left(\mathcal{F}_{A l-4}\right)
$$

$$
\mathcal{F}_{A l-5} \geq \mathcal{F}_{A l-3} \geq \mathcal{F}_{A l-2} \geq \mathcal{F}_{A l-1} \geq \mathcal{F}_{A l-4}
$$

Therefore, from the above analysis, we get the result the alternative $\mathcal{F}_{A l-5}$ is the best optimal.

In Example 1, we choose the complex interval-valued Pythagorean fuzzy sorts of information and resolve it by using the elaborated CIVPFEWG operator. Further, to determine the reliability and dominance of the elaborated operators, we choose some prevailing sorts of information and resolve it by using elaborated operators to show the dominance and consistency of the elaborated works.

Example 2: (Garg, and Rani, 2019a) This theory is taken from Ref. (Garg, and Rani, 2019a), which states that the utilization of the created approach, we delineate a model. Assume a business visionary chooses to buy another machine for his organization from a machine creator $\mathbb{X}_{u n i}$. The machine producer gives data on four models of machine $\left(\mathcal{F}_{A l-1}, \mathcal{F}_{A l-2}, \mathcal{F}_{A l-3}, \mathcal{F}_{A l-4}\right)$ with various creation dates for each model and their attributes are in the form of selection experts whose expressions are followed as:

$\mathcal{F}_{A t-1}$ : Expressed the reliability.

$\mathcal{F}_{A t-2}$ : Expressed the safety.

$\mathcal{F}_{A t-3}$ : Expressed the flexibility.

$\mathcal{F}_{A t-4}$ : Expressed productivity.

To determine the above troubles, we choose the family of weight vectors such that $0.3,0.2,0.1,0.4$, Then by using the above family of $n$ alternatives and $m$ attributes, we apply the steps of the algorithm of the proposed work on Table 1 in Ref. (Garg, and Rani, 2019a). The aggregated results are discussed below.

$$
\begin{gathered}
\mathcal{F}_{A l-1}=\operatorname{CIVPFEWG}\left(\mathcal{F}_{A t-1}, \mathcal{F}_{A t-2}, \mathcal{F}_{A t-3}, \mathcal{F}_{A t-4}\right)=\left(\begin{array}{c}
{[0.2554,0.4233] e^{i 2 \pi([0.2851,0.4566])},} \\
\left.[0.2051,0.3569] e^{i 2 \pi([0.3217,0.4842])}\right)
\end{array}\right) \\
\mathcal{F}_{A l-2}=\operatorname{CIVPFEWG}\left(\mathcal{F}_{A t-1}, \mathcal{F}_{A t-2}, \mathcal{F}_{A t-3}, \mathcal{F}_{A t-4}\right)=\left(\begin{array}{c}
{[0.2943,0.4981] e^{i 2 \pi([0.292,0.5679])},} \\
{[0.114,0.2346] e^{i 2 \pi([0.1,0.2647])}}
\end{array}\right)
\end{gathered}
$$




$$
\begin{gathered}
\mathcal{F}_{A l-3}=\operatorname{CIVPFEWG}\left(\mathcal{F}_{A t-1}, \mathcal{F}_{A t-2}, \mathcal{F}_{A t-3}, \mathcal{F}_{A t-4}\right)=\left(\begin{array}{c}
{[0.337,0.4878] e^{i 2 \pi([0.2858,0.5586])},} \\
\left.[0.1581,0.3055] e^{i 2 \pi([0.1379,0.2647])}\right)
\end{array}\right) \\
\mathcal{F}_{A l-4}=\operatorname{CIVPFEWG}\left(\mathcal{F}_{A t-1}, \mathcal{F}_{A t-2}, \mathcal{F}_{A t-3}, \mathcal{F}_{A t-4}\right)=\left(\begin{array}{l}
{[0.2225,0.5421] e^{i 2 \pi([0.1538,0.3431])},} \\
{[0.1483,0.2647] e^{i 2 \pi([0.1761,0.4248])}}
\end{array}\right)
\end{gathered}
$$

By using the score function, we find the Score values of the aggregated values, which are discussed below.

$$
\mathbb{S}^{s f}\left(\mathcal{F}_{A l-1}\right)=0.0134, \mathbb{S}^{s f}\left(\mathcal{F}_{A l-2}\right)=0.2972, \mathbb{S}^{s f}\left(\mathcal{F}_{A l-3}\right)=0.2689, \mathbb{S}^{s f}\left(\mathcal{F}_{A l-4}\right)=0.0906,
$$

By using the above Score values, we rank all alternatives and examine the best one such that

$$
\mathbb{S}^{s f}\left(\mathcal{F}_{A l-2}\right) \geq \mathbb{S}^{s f}\left(\mathcal{F}_{A l-3}\right) \geq \mathbb{S}^{s f}\left(\mathcal{F}_{A l-4}\right) \geq \mathbb{S}^{s f}\left(\mathcal{F}_{A l-1}\right)
$$

That means that

$$
\mathcal{F}_{A l-2} \geq \mathcal{F}_{A l-3} \geq \mathcal{F}_{A l-4} \geq \mathcal{F}_{A l-1}
$$

Therefore, from the above analysis, we get the result the alternative $\mathcal{F}_{A l-2}$ is the best optimal.

In Example 1, we choose the complex interval-valued Pythagorean fuzzy sorts of information and resolve it by using the elaborated CIVPFEWG operator, and in Example 2, we choose the complex interval-valued intuitionistic fuzzy sorts of information and resolve it by using the elaborated CIVPFEWG operator. Further, to determine the reliability and dominance of the elaborated operators, we choose some prevailing operators and compare them with proposed operators by using the information of Example 1 and Example 2, to show the dominance and consistency of the elaborated works.

Certain people have elaborated on different sorts of operators based on IVIFSs, IVPFSs, CIVIFSs and compare our results with some existing operators to determine the supremacy of their works. The goal of this study, we determine the practicability and dominance of the elaborated operator with the help of comparison with some prevailing operators to find the consistency and flexibility of the developed operator based on novel CIVPFSs. We compare the proposed operator with some prevailing operators whose information's are follow as Wang et al. (2012) elaborated aggregation operators for IVIFSs, Haktanır (2020) initiated the theory of aggregation operators for IVPFSs, Garg and Rani (2019) proposed aggregation operators for CIVIFSs, and with proposed operators to examine the dominance and supremacy of the elaborated operator. Based on the information in Table 1, the comparative analysis of the elaborated operator with some prevailing operators is implemented in the form of Table 2.

Table 2. Comparative analysis between proposed and existing operators.

\begin{tabular}{lcc}
\hline Method & Score Values & Ranking Results \\
\hline Wang et al. (2012) & Cannot be Calculated & Cannot be Calculated \\
\hline Haktanır (2020) & Cannot be Calculated & Cannot be Calculated \\
\hline $\begin{array}{l}\text { Garg and Rani } \\
\text { (2019a) }\end{array}$ & Cannot be Calculated & Cannot be Calculated \\
\hline $\begin{array}{l}\text { CIVPFEWG } \\
\text { operator }\end{array}$ & $\mathbb{S}^{s f}\left(\mathcal{F}_{A l-1}\right)=0.1981, \mathbb{S}^{s f}\left(\mathcal{F}_{A l-2}\right)$ & $=0.2420$, \\
& $\mathfrak{S}^{s f}\left(\mathcal{F}_{A l-3}\right)=0.3473, \mathbb{S}^{s f}\left(\mathcal{F}_{A l-4}\right)$ & \\
& $=0.0868$, & \\
& $\mathbb{S}^{s f}\left(\mathcal{F}_{A l-5}\right)=0.5009$ & \\
\end{tabular}

From the above investigation's we know that if we choose the information in the form of complex interval-valued Pythagorean fuzzy numbers, then the prevailing operators are based on IVIFSs (Wang et al., 2012), IVPFSs (Haktanır, 2020), and CIVIFSs (Garg and Rani, 2019a) are not able to cope with it. Further, we show that if we choose the information in the form of complex interval-valued intuitionistic fuzzy numbers then the theory of Garg and Rani (2019a) can resolve it, but still, the theory of IVIFSs (Wang et al., 2012), IVPFSs (Haktanır, 2020) are failed. Based on the information of Table 1 from Ref. (Garg and Rani, 2019a), the comparative analysis of the elaborated operator with some prevailing operators is implemented in the form of Table 3 . 
Table 3. Comparative analysis between proposed and existing operators.

\begin{tabular}{|c|c|c|}
\hline Method & Score Values & Ranking Results \\
\hline $\begin{array}{l}\text { Wang et al. } \\
\text { (2012) }\end{array}$ & Cannot be Calculated & Cannot be Calculated \\
\hline Haktanır (2020) & Cannot be Calculated & Cannot be Calculated \\
\hline $\begin{array}{l}\text { Garg and Rani } \\
(2019 a)\end{array}$ & $\begin{array}{c}\mathbb{S}^{s f}\left(\mathcal{F}_{A l-1}\right)=0.0023, \mathbb{S}^{s f}\left(\mathcal{F}_{A l-2}\right) \\
=0.1861 \\
\mathbb{S}^{s f}\left(\mathcal{F}_{A l-3}\right)=0.1578, \mathbb{S}^{s f}\left(\mathcal{F}_{A l-4}\right) \\
=0.0805\end{array}$ & $\mathcal{F}_{A l-2} \geq \mathcal{F}_{A l-3} \geq \mathcal{F}_{A l-4} \geq \mathcal{F}_{A l-1}$ \\
\hline $\begin{array}{l}\text { CIVPFEWG } \\
\text { operator }\end{array}$ & $\begin{array}{c}\mathbb{S}^{s f}\left(\mathcal{F}_{A l-1}\right)=0.0134, \mathbb{S}^{s f}\left(\mathcal{F}_{A l-2}\right) \\
=0.2972 \\
\mathbb{S}^{s f}\left(\mathcal{F}_{A l-3}\right)=0.2689, \mathbb{S}^{s f}\left(\mathcal{F}_{A l-4}\right) \\
=0.0906\end{array}$ & $\mathcal{F}_{A l-2} \geq \mathcal{F}_{A l-3} \geq \mathcal{F}_{A l-4} \geq \mathcal{F}_{A l-1}$ \\
\hline
\end{tabular}

As shown above, the elaborated operators based on CIVPFSs are more important and powerful than the existing theory (Wang et al., 2012; Garg and Rani, 2019a; Haktanır, 2020) to manage inconsistent and unreliable information in genuine life troubles.

\section{Conclusion}

The principle of CIVPFS is a valuable procedure to manage inconsistent and awkward information genuine life troubles. The principle of CIVPFS is a mixture of the two separated theories such as the complex fuzzy set and interval-valued Pythagorean fuzzy set which covers the TG and FG in the form of the complex number whose real and unreal parts are the sub-interval of the unit interval. The superiority of the CIVPFS is that the sum of the square of the upper grade of the real part (also for the unreal part) of the duplet is restricted to the unit interval. The goal of this article is to explore the new principle of CIVPFS and its algebraic operational laws. By using the CIVPFSs, certain Einstein operational laws by using the t-norm and t-conorm are also developed. Additionally, we explore the CIVPFEWG, CIVPFEOWG operators and utilized their special cases. Moreover, an MCDM technique is explored based on the elaborated operators by using the CIVPF information. To determine the consistency and reliability of the elaborated operators, we illustrated certain examples by using the explored principles. Finally, to determine the supremacy and dominance of the explored theories, the comparative analysis and graphical expressions of the developed principles are also discussed.

In the future, we will extend the elaborated work for complex q-rung orthopair fuzzy sets (Ali and Mahmood, 2020), complex spherical fuzzy sets (Ali et al., 2020a), and complex T-spherical fuzzy sets (Ali et al., 2020b) to improve the quality of the research works.

\section{References}

Ali, M., Tamir, D. E., Rishe, N. D., \& Kandel, A. (2016). Complex intuitionistic fuzzy classes. In 2016 IEEE International Conference on Fuzzy Systems (FUZZ-IEEE) (pp. 2027-2034). IEEE.

Ali, Z., \& Mahmood, T. (2020). Maclaurin symmetric mean operators and their applications in the environment of complex q-rung orthopair fuzzy sets. Computational and Applied Mathematics, 39, 1-27.

Ali, Z., Mahmood, T., \& Yang, M. S. (2020a). TOPSIS Method Based on Complex Spherical Fuzzy Sets with Bonferroni Mean Operators. Mathematics, 8(10), 1739.

Ali, Z., Mahmood, T., \& Yang, M. S. (2020b). Complex T-spherical fuzzy aggregation operators with application to multi-attribute decision making. Symmetry, 12(8), 1311.

Alkouri, A. M. D. J. S., \& Salleh, A. R. (2012, September). Complex intuitionistic fuzzy sets. In AIP conference proceedings (Vol. 1482, No. 1, pp. 464-470). American Institute of Physics.

Atanassov, K. (1986). Intuitionistic fuzzy sets. fuzzy sets and systems 20 (1), 87-96.

Atanassov, K., \& Gargov, G. (1989). Interval valued intuitionistic fuzzy sets, Fuzzy Sets and Systems 31, 343-349.

Aydın, T., \& Enginoğlu, S. (2021). Interval-valued intuitionistic fuzzy parameterized interval-valued intuitionistic fuzzy soft sets and their application in decision-making. Journal of Ambient Intelligence and 
Humanized Computing, 12(1), 1541-1558.

Ayyildiz, E., \& Gumus, A. T. (2021). Interval-valued Pythagorean fuzzy AHP method-based supply chain performance evaluation by a new extension of SCOR model: SCOR 4.0. Complex \& Intelligent Systems, 7(1), 559-576.

Çalık, A. (2021). A novel Pythagorean fuzzy AHP and fuzzy TOPSIS methodology for green supplier selection in the Industry 4.0 era. Soft Computing, 25(3), 2253-2265.

Chen, T. Y. (2021). The likelihood-based optimization ordering model for multiple criteria group decision making with Pythagorean fuzzy uncertainty. Neural Computing and Applications, 33(10), 4865-4900.

Ecer, F., \& Pamucar, D. (2021). MARCOS technique under intuitionistic fuzzy environment for determining the COVID-19 pandemic performance of insurance companies in terms of healthcare services. Applied Soft Computing, 104, 107199.

Ejegwa, P. A., \& Onyeke, I. C. (2021). Intuitionistic fuzzy statistical correlation algorithm with applications to multicriteria-based decision-making processes. International Journal of Intelligent Systems, 36(3), 1386-1407.

Ejegwa, P., Wen, S., Feng, Y., Zhang, W., \& Tang, N. (2021). Novel Pythagorean fuzzy correlation measures via Pythagorean fuzzy deviation, variance and covariance with applications to pattern recognition and career placement. IEEE Transactions on Fuzzy Systems.

Gao, X., Pan, L., \& Deng, Y. (2021). Quantum pythagorean fuzzy evidence theory (qpfet): A negation of quantum mass function view. IEEE Transactions on Fuzzy Systems.

Garg, H. (2016). A novel accuracy function under interval-valued Pythagorean fuzzy environment for solving multicriteria decision making problem. Journal of Intelligent \& Fuzzy Systems, 31(1), 529-540.

Garg, H. (2017). Generalized Pythagorean fuzzy geometric aggregation operators using Einstein t-norm and $\mathrm{t}$-conorm for multicriteria decision-making process. International Journal of Intelligent Systems, 32(6), 597-630.

Garg, H., \& Rani, D. (2019a). Complex interval-valued intuitionistic fuzzy sets and their aggregation operators. Fundamenta Informaticae, 164(1), 61-101.

Garg, H., \& Rani, D. (2019b). Some results on information measures for complex intuitionistic fuzzy sets. International Journal of Intelligent Systems, 34(10), 2319-2363.

Garg, H., \& Rani, D. (2019c). A robust correlation coefficient measure of complex intuitionistic fuzzy sets and their applications in decision-making. Applied intelligence, 49(2), 496-512.

Garg, H., \& Rani, D. (2019d). Generalized geometric aggregation operators based on t-norm operations for complex intuitionistic fuzzy sets and their application to decision-making. Cognitive Computation, 1-20.

Garg, H., \& Rani, D. (2021). Novel similarity measure based on the transformed right-angled triangles between intuitionistic fuzzy sets and its applications. Cognitive Computation, 13(2), 447-465.

Haktanır, E. (2020). Interval valued pythagorean fuzzy aggregation operators based malcolm baldrige national quality award assessment. Journal of Intelligent \& Fuzzy Systems, (Preprint), 1-11.

Huang, W., Zhang, F., \& Xu, S. (2021). A complete ranking method for interval-valued intuitionistic fuzzy numbers and its applications to multicriteria decision making. Soft Computing, 25(3), 2513-2520.

Karnik, N. N., \& Mendel, J. M. (2001). Operations on type-2 fuzzy sets. Fuzzy sets and systems, 122(2), 327-348.

Kumar, T., \& Bajaj, R. K. (2014). On complex intuitionistic fuzzy soft sets with distance measures and entropies. Journal of Mathematics, 2014.

Mahmood, T. (2020). A Novel Approach towards Bipolar Soft Sets and Their Applications. Journal of Mathematics, 2020.

Mendel, J. M., \& John, R. B. (2002). Type-2 fuzzy sets made simple. IEEE Transactions on fuzzy systems, 10(2), 117-127.

Ngan, R. T., Ali, M., Tamir, D. E., Rishe, N. D., \& Kandel, A. (2020). Representing complex intuitionistic fuzzy set by quaternion numbers and applications to decision making. Applied Soft Computing, 87, 105961.

Pan, L., Gao, X., Deng, Y., \& Cheong, K. H. (2021). The constrained pythagorean fuzzy sets and its similarity measure. IEEE Transactions on Fuzzy Systems.

Ramot, D., Milo, R., Friedman, M., \& Kandel, A. (2002). Complex fuzzy sets. IEEE Transactions on Fuzzy Systems, 10(2), 171-186. 
Rani, D., \& Garg, H. (2018). Complex intuitionistic fuzzy power aggregation operators and their applications in multicriteria decision-making. Expert Systems, 35(6), e12325.

Rani, P., Mishra, A. R., Saha, A., \& Pamucar, D. (2021). Pythagorean fuzzy weighted discrimination-based approximation approach to the assessment of sustainable bioenergy technologies for agricultural residues. International Journal of Intelligent Systems, 36(6), 2964-2990.

Torra, V. (2010). Hesitant fuzzy sets. International Journal of Intelligent Systems, 25(6), 529-539.

Ullah, K., Mahmood, T., Ali, Z., \& Jan, N. (2020). On some distance measures of complex Pythagorean fuzzy sets and their applications in pattern recognition. Complex \& Intelligent Systems, 6(1), 15-27.

Wang, W., \& Liu, X. (2011). Intuitionistic fuzzy geometric aggregation operators based on Einstein operations. International Journal of Intelligent Systems, 26(11), 1049-1075.

Wang, W., \& Liu, X. (2012). Intuitionistic fuzzy information aggregation using Einstein operations. IEEE Transactions on Fuzzy Systems, 20(5), 923-938.

Wang, W., Liu, X., \& Qin, Y. (2012). Interval-valued intuitionistic fuzzy aggregation operators. Journal of Systems Engineering and Electronics, 23(4), 574-580.

Xue, Y., Deng, Y., \& Garg, H. (2021). Uncertain database retrieval with measure-Based belief function attribute values under intuitionistic fuzzy set. Information Sciences, 546, 436-447.

Yager, R.R. (2013), June. Pythagorean fuzzy subsets. In 2013 joint IFSA world congress and NAFIPS annual meeting (IFSA/NAFIPS) (pp. 57-61). IEEE.

Zadeh, L. A. (1965). Information and control. Fuzzy sets, 8(3), 338-353.

Zadeh, L. A. (1975). The concept of a linguistic variable and its application to approximate reasoningI. Information sciences, 8(3), 199-249.

Zhao, M., Wei, G., Wei, C., \& Wu, J. (2021). TODIM method for interval-valued Pythagorean fuzzy MAGDM based on cumulative prospect theory and its application to green supplier selection. Arabian Journal for Science and Engineering, 46(2), 1899-1910.

Zulqarnain, R. M., Siddique, I., Jarad, F., Ali, R., \& Abdeljawad, T. (2021). Development of TOPSIS Technique under Pythagorean Fuzzy Hypersoft Environment Based on Correlation Coefficient and Its Application towards the Selection of Antivirus Mask in COVID-19 Pandemic. Complexity, 2021. 